University of Louisville

ThinkIR: The University of Louisville's Institutional Repository

Electronic Theses and Dissertations

$5-2015$

\title{
The evolution and ecology of individual specializations amongst a group of dietary generalists.
}

Carl S. Cloyed

University of Louisville

Follow this and additional works at: https://ir.library.louisville.edu/etd

Part of the Biology Commons

\section{Recommended Citation}

Cloyed, Carl S., "The evolution and ecology of individual specializations amongst a group of dietary generalists." (2015). Electronic Theses and Dissertations. Paper 2105.

https://doi.org/10.18297/etd/2105

This Doctoral Dissertation is brought to you for free and open access by ThinkIR: The University of Louisville's Institutional Repository. It has been accepted for inclusion in Electronic Theses and Dissertations by an authorized administrator of ThinkIR: The University of Louisville's Institutional Repository. This title appears here courtesy of the author, who has retained all other copyrights. For more information, please contact thinkir@louisville.edu. 
THE EVOLUTION AND ECOLOGY OF INDIVIDUAL SPECIALIZATIONS AMONGST A GROUP OF DIETARY GENERALISTS

\author{
By \\ Carl S. Cloyed \\ B.A. Prescott College 2008

\begin{abstract}
A Dissertation
Submitted to the Faculty of the

College of Arts and Sciences of the University of Louisville in Partial Fulfillment of the Requirements

for the Degree of
\end{abstract}

\author{
Doctor of Philosophy \\ in Biology \\ Department of Biology \\ University of Louisville \\ Louisville, Kentucky
}

May 2015 
Copyright 2015 by Carl Stephen Cloyed

All rights reserved 

THE EVOLUTION AND ECOLOGY OF INDIVIDUAL SPECIALIZATIONS AMONGST A GROUP OF DIETARY GENERALISTS

By

Carl S. Cloyed

B.A. Prescott College 2008

A Dissertation Approved on

April 3, 2015

by the Following Dissertation Committee:

\begin{tabular}{c}
\hline $\begin{array}{c}\text { Dissertation Director } \\
\text { Perri K. Eason }\end{array}$ \\
\hline Lee Dugatkin \\
\hline Sarah Emery \\
\hline Robert Page \\
\hline Seth D. Newsome
\end{tabular}




\section{ACKNOWLEDGMENTS}

I would like to thank Jeff Masters, Jennifer Siow, Troy Tucker, Seth Quinn, Gavin Johnson, and Amber Quaack for field help; Bernheim Research Arboretum and Research Forest and Horner Biological Reserve for use of the study area; and the Kentucky Society for Natural History and the University of Louisville School and Arts and Sciences for funding. I would especially like to thank my parents, Craig and Susan Cloyed, and my partner Jennifer Siow for all the love and support they have given to me. 


\title{
ABSTRACT \\ THE EVOLUTION AND ECOLOGY OF INDIVIDUAL SPECIALIZATIONS AMONGST A GROUP OF DIETARY GENERALISTS
}

\author{
Carl S. Cloyed
}

April 3, 2015

This dissertation examines individual diet specializations (IS) in a group of ecologically similar and evolutionarily related frogs and toads. Individual specialization is known to have widespread ecological and evolutionary effects.

In an initial literature review (Chapter 2) I build a comprehensive theoretical framework showing how different types of population diversity can help, halt, or hinder sympatric speciation. I argue that IS can be maintained indefinitely in populations yet fail to lead to speciation because it is influenced by ecological conditions that may change. Additionally, IS can potentially aid niche partitioning among similar species, increasing species coexistence and resulting in less of the ecological opportunity required to develop more discrete polymorphisms.

Stable isotopes are an increasingly common ecological tool for determining diets and habitat usage. However, to use them accurately, researchers need taxon-specific trophic discrimination factors and isotopic incorporation rates on any tissue used for stable isotope analysis. I determined these important isotope properties in adult frogs for the first time (Chapter 3), which not only allows me to use them in the following sections of 
my dissertation, but also allows other researchers to use stable isotopes to study frog and toad diets.

Using stable isotope analyses, I examined how IS is influenced by ecological conditions (Chapter 4). I measured IS in five species of frogs and toads and determined which of three ecological parameters (resource diversity, intraspecific competition, and interspecific competition) affected IS in each species. I found that species differed in which ecological parameter best explained IS. Resource diversity most frequently affected IS, with conspecific density second in importance. My results showed that different ecological conditions support IS in different species.

Finally, again using stable isotopes, I investigated whether intrapopulation niche variation could aid niche partitioning among the same five species of frogs and toads (Chapter 5). I found that species differed in their niches, but that subsets of individuals overlapped among species. The limited number of individuals overlapping between species decreases their interaction strength, which can contribute to niche partitioning and thus to species coexistence. 
TABLE OF CONTENTS

PAGE

ACKNOWLEDGMENTS

ABSTRACT iv

LIST OF TABLES viii

LIST OF FIGURES ix

CHAPTER I: INTRODUCTION ORGANIZATION OF DISSERTATION 1 SPECIATION

SUMMARY 7

INTRODUCTION 8 CONCEPTUAL MODEL

SPECIATION TRAPS

SPECIATION OBSTICLES

SPECIATION

CHAPTER III: TROPHIC DISCRIMINATION FACTORS AND INCORPORATION RATES OF CARBON AND NITROGEN STABLE ISOTOPES IN ADULT GREEN FROGS, LITHOBATES CLAMITANS

$\begin{array}{lr}\text { SUMMARY } & 51 \\ \text { INTRODUCTION } & 52 \\ \text { METHODS } & 55 \\ \text { RESULTS } & 60 \\ \text { DISCUSSION } & 62\end{array}$

CHAPTER IV: DIFFERENT ECOLOGICAL CONDITIONS SUPPORT INTRAPOPULATION NICHE VARIATION IN CLOSELY RELATED AND ECOLOGICALLY SIMILAR SPECIES 76 


$\begin{array}{lr}\text { SUMMARY } & 76 \\ \text { INTRODUCTION } & 77 \\ \text { METHODS } & 81 \\ \text { RESULTS } & 88 \\ \text { DISCUSSION } & 91\end{array}$

CHAPTER V: RESOURCE PARTITIONING AND THE ROLE OF INTRAPOPULATION NICHE VARIATION IN STRUCTURING A GUILD OF GENERALIST ANURANS

SUMMARY 115

INTRODUCTION

METHODS

RESULTS

124

DISCUSSION

CHAPTER VI: SUMMARY AND FUTURE DIRECTIONS 146

SUMMARY 148

FUTURE DIRECTIONS

REFERENCES

CURRICULUM VITAE 


\section{LIST OF TABLES}

PAGE

1. Differences between Migration-Selection and Heterozygotic Protected Polymorphisms 38

2. Resource Polymorphisms Arising from Phenotypic Plasticity And Genetic Mutations 39

3. Sexual Dimorphisms with Ecological Consequences 41

4. Isotopic Incorporation Equations of Best Fit, Half Lives, and Trophic Discrimination Factors for Carbon and Nitrogen in Skin, Whole Blood, and Bone Collagen 70

5. Mean $\delta^{13} \mathrm{C}$ and $\delta^{15} \mathrm{~N}$ Values in Skin, Whole Blood, and Bone Collagen 71

6. $\mathrm{AIC}_{\mathrm{c}}$ and $\Delta \mathrm{AIC}_{\mathrm{c}}$ values for each model across both isotope types and all tissues types 72

7.The average proportional dissimilarity $(\mathrm{PD}) \pm$ standard deveiation $(\mathrm{SD})$ at each pond for each species 101

8. Ecological parameter models for each species 103

9. The parameter weights, averaged beta, and $95 \% \mathrm{CI}$ of the averaged beta 105

10. Mean proportion $( \pm \mathrm{SD})$ of each prey group by site 138

11. Results from between species permutation tests of Chesson's Alpha Selectivity index 139 


\section{LIST OF FIGURES}

PAGE

1. Conceptual Framework of Sympatric Speciation___ 48

2. Opposing Directional Selection between Sexes__ 49

3. Ecological Parameters that Affect Individual Diet Specializations __ 50

4. Isotopic Incorporation Curves and Reaction Prgrees Variable for Carbon__ 74

5. Isotopic Incorporation Curves and Reaction Prgrees Variable for Nitrogen_ 75

6. Correlation between Stable Isotopes in Skin and Bone Collagen___ 108

7. $\delta^{13} \mathrm{C}$ and $\delta^{15} \mathrm{~N}$ Biplot for Taods___ 109

8. $\delta^{13} \mathrm{C}$ and $\delta^{15} \mathrm{~N}$ Biplot for True Frogs___ 110

9. Resource Diversity versus Proportional Dissimilarity____ 111

10. Conspecific Density versus Proportional Dissimilarity___ 112

11. Heterospecific Density versus Proportional Dissimilarity___ 113

12. Barplot with proportional dissimilarity values measured with SIA and SCA_114

13. Snout-Vent Length and Distance to Pond by Species____ 142

14. Stable Isotope Biplot with Mean of Each Species___ 143

15. $\delta^{15} \mathrm{~N}$ and $\delta^{13} \mathrm{C}$ Values by Species___ 144

16. $\delta^{15} \mathrm{~N}$ and $\delta^{13} \mathrm{C}$ Values by Snout-Vent Length___ 145

17. $\delta^{15} \mathrm{~N}$ and $\delta^{13} \mathrm{C}$ Values by Snout-Vent Length by Site for Each Species___ 146

18. Mean Chesson's Alpha for Each Prey Group on all Species___ 147 


\section{CHAPTER I}

\section{INTRODUCTION}

Ecological studies have traditionally treated individuals equivalently. However, intrapopulation niche variation can have profound effects on populations and communities (Tinker et al. 2008; Schindler et al. 2010; Bolnick et al. 2011; Wennersten and Forsman 2012), and is an important source of variation upon which natural selection can act (Schluter 2000, 2001). For example, many species that are considered generalist foragers are actually composed of individuals that only use a subset of the species' resource spectrum, a phenomenon known as individual specialization (IS; Bolnick et al. 2003; Wennersten and Forsman 2012). Individual specialization can decrease intraspecific competition (Svanbäck and Bolnick 2007; Tinker et al. 2008), decrease extinction rates (Schindler et al. 2010), and alter the connectivity of energy and nutrients among habitats (Quevedo et al. 2009; Rosenblatt and Heithaus 2011). Finally, individual specializations are thought to be the variation upon which natural selection can act to develop more discrete resource polymorphisms (Schluter 2000; Nosil 2012). These polymorphisms are candidates for speciation events in sympatry.

There has been a longstanding debate in ecology and evolutionary biology about the rarity of sympatric speciation. Proponents of sympatric speciation have had recent theoretical and empirical breakthroughs. For example, theoretical evolutionary biologists have determined how sympatric speciation can occur in the presence of frequency- 
dependent selection (Seger 1985; Doebeli 1996; Dieckmann and Doebeli 1999; Doebeli et al. 2007), and furthermore, field-based studies have demonstrated that several speciation events have occurred in sympatry (Schliewen et al. 1994; Feder et al. 2003; Barluenga et al. 2006; Ryan et al. 2007; Foote et al. 2009; Crow et al. 2010; Kautt et al. 2012). However, despite these breakthroughs, it appears that sympatric speciation is still uncommon compared to allopatric speciation (Coyne and Price 2000; Ribera et al. 2001; Fitzpatrick and Turelli 2006; Grant and Grant 2008). One possible explanation for this is that when population level diversity arises, it may result in several alternative evolutionary states (Rueffler et al. 2006), and some of these states can prevent speciation.

Among these different evolutionary states are individual specializations, sexual dimorphisms, polymorphisms caused by heterozygotic advantage, polymorphisms that are caused by phenotypic plasticity, and genetic polymorphisms not caused by heterozygotic advantage. Individual specializations are thought to initiate sympatric speciation and to lead to more discrete genetic polymorphisms. However, they appear to be quite common (Bolnick et al. 2003; Araújo et al. 2011), and many of them likely do not lead to more discrete polymorphisms and to sympatric speciation.

Individual specializations may not ever become discrete polymorphisms for two reasons. First, many individual specializations are often influenced by ecological conditions, and these conditions can change over time. For example, IS is greater when intraspecific competition is high (Svanäck and Bolnick 2007; Agashe and Bolnick 2010; Frédérich et al. 2010; Tinker et al. 2012; Evangelista et al. 2014), when interspecific competition is low (Bolnick et al. 2003, 2011; however see Bolnick et al. 2010: AbbeyLee 2013), and when some form of ecological opportunity is available (Nosil and 
Reimchen 2005; Parent and Crespi 2009; Darimont et al. 2009). These ecological parameters that influence IS are likely to change over time, and populations can move between being composed of individual specialists versus of individual generalists (Tinker et al. 2008). Second, individual specializations may increase species coexistence by decreasing the interaction strength among competing heterospecifics (Lichstein et al. 2007; Bolnick et al. 2011). This increase in species coexistence can decrease the likelihood that discrete polymorphisms will develop because more ecologically similar species in a community will utilize the ecological opportunity that developing polymorphisms require (Losos 2010).

\section{ORGANIZATION OF DISSERTATION}

The focus of this dissertation is to examine the ecological and evolutionary roles of individual specialization and the ecological causes of it. I examine this both from a conceptual approach and by studying the individual diet patterns across five species of frogs and toads: Anaxyrus americanus, A.fowleri, Lithobates catesbeianus, L. clamitans, and L. sphenocephalus. The dissertation is broken into four sections.

The first section (chapter two) is an intensive review that constructs a conceptual framework that explains why sympatric speciation is difficult and how different types of population diversity can prevent or hinder speciation. Sexual dimorphisms and polymorphisms created by heterozygotic advantage will halt speciation along an ecological trait. Individual specialization and disruptive selection on other types of genetically produced resource polymorphisms can hinder sympatric speciation if 
ecological conditions are inconsistent or if assortative mating fails to develop and generate reproductive isolation. It is in this review chapter that I outline how individual specializations can prevent the development of discrete polymorphisms. In Chapter 3 of my dissertation I develop tools that I use in Chapters 4 and 5 to investigate the ecological causes and consequences of these individual specializations (IS). I help determine these two ecological and evolutionary effects of IS in chapters 4 and 5 of this dissertation.

The second section (chapter three) is a methods chapter in which I performed two controlled feeding experiments to determine important stable isotope properties, trophic discrimination factors and isotopic incorporation rates, in adult frogs. I determined these values for both carbon and nitrogen isotopes in skin, whole blood, and bone collagen in adult green frogs, L. clamitans. While many studies have determined these taxon- and sometimes species-specific properties, none have been performed on adult anurans and very few have been performed on amphibians (Caut et al. 2013). This study will enable researchers to determine diet and habitat use across short and long timeframes. Given the rate of extinction and population declines in many anuran species, this information will prove invaluable to many researchers and land mangers. Furthermore, I use both trophic discrimination factors and isotopic incorporation rates in the third and fourth sections of my dissertation.

In Chapter four, I determined the degree of IS in the five abovementioned frogs and toads and tested how different ecological parameters affect IS in each species. I determined proportions of prey types for each individual using a Bayesian stable isotope mixing model and used these proportions to determine within population variability. I tested how this within-population variability changed at sites with different resource 
diversity, conspecific density, and heterospecific density. In this section, I showed that these different ecological parameters affect IS and that species differ in which parameters affect IS and in the strength of those effects. It is these ecological parameters that influence IS and may not stay consistent over time, forestalling the development of discrete polymorphisms.

In Chapter 5, I test niche partitioning among the five species of frogs and toads as well as how intrapopulation niche variation can contribute to that niche partitioning. I test how species differ in their nitrogen and carbon isotopes, as well as how species differ in several morphological traits and in habitat choice. Higher values of carbon isotopes were associated with terrestrial prey and habitats, while lower values indicated aquatic prey and habitats. High values of nitrogen isotopes indicated higher trophic level. I found that species varied in diet, trophic level, and in habitat choice. These differences were confirmed with stable isotopes, stomach contents, and comparisons of where frogs and toads were captured in relation to pond edges. Furthermore, trophic level increased within species as individuals became larger. This ontogenetic change in trophic level altered the interaction strength between several heterospecific species pairs, thus aiding in niche partitioning.

In the final chapter of my dissertation I summarize my findings. I also reveal several future directions. In these future directions I will include a framework for performing more empirical studies to test hypotheses generated in the first section. I will then go into ways to help solidify the role of IS as both a starting point for the development of resource polymorphisms and sympatric speciation as well as an important ecological trait 
that may persist indefinitely in populations without ever developing into discrete polymorphisms. 


\section{CHAPTER II}

\section{THE EFFECTS OF POPULATION DIVERSITY ON SYMPATRIC SPECIATION}

\section{SUMMARY}

The debate regarding the role of geography in speciation is long-standing, contentious, and ongoing. In general, sympatric speciation is considered rare and allopatric conditions are more likely to lead to divergence. The following review explains why sympatric speciation is rare. We lay out how ecological, sympatric speciation can occur along two axes, degree of divergence in the ecological trait and reproductive isolation. We map the types of intrapopulation niche variation that can be generated. These different types of variation include individual specializations, ecologically reinforced sexual dimorphisms, heterozygotic polymorphisms, and sympatrically cladogenic polymorphisms. We categorize these types of variation into speciation traps and speciation obstacles. Speciation traps halt the process of speciation in the trait under selection and include ecologically reinforced sexual dimorphisms and heterozygotic polymorphisms. Speciation traps increase population diversity but can decrease the potential number of species in an area because they inhibit speciation. Speciation obstacles, on the other hand, are processes that can slow and potentially halt speciation in otherwise sympatrically cladogenic polymorphisms. These speciation obstacles include 
cycles of specialization and disruptive selection as well as the special problem of developing assortative mating and the resulting reproductive isolation. Finally, we determine what conditions are needed to increase the likelihood that ecological speciation happens in sympatry.

\section{INTRODUCTION}

Ever since Mayr (1963) declared sympatric speciation impossible for theoretical and genetic reasons, biologists have been arguing about the frequency and circumstances of its occurrence (Maynard-Smith 1966; Futuyma and Mayer 1980; Seger 1985; Dieckmann and Doebeli 1999; Coyne and Orr 2004). There is general agreement that speciation is more common in allopatry than in sympatry (Coyne and Price 2000; Ribera et al. 2001; Fitzpatrick and Turelli 2006; Grant and Grant 2008). Most researchers have considered sympatric speciation to be extremely rare and only influential in small, isolated environments (Schliewen et al. 1994; Schluter and Rambaut 1996; Barluenga et al. 2006; Ryan et al. 2007; Bolnick and Fitzpatrick 2007; Kuatt et al. 2012) or in host races of phytophageous insects (Walsh 1864; Drès and Mallet 2002; Berlocher and Feder 2002). Some researchers have even suggested abandoning the geographic categorization of speciation (Butlin et al. 2008; Fitzpatrick et al. 2009). However, geography plays an important role in speciation because speciation can occur through either genetic drift or selection in geographic isolation, whereas only selection can produce divergence in sympatry (Coyne and Orr 2004; Mallet et al. 2009). Further, some theoreticians have argued that although sympatric speciation is relatively rare, it may be more frequent than commonly believed (Maynard-Smith 1966; Rosenzweig 1978; Seger 1985; Doebeli 
1996; Dieckmann and Doebeli 1999; Doebeli et al. 2007). Recent empirical studies have provided support for these theoretical arguments by demonstrating that sympatric speciation can also occur in large, well-connected environments such as oceans (Crow et al. 2010; Foote et al. 2009; Wolf et al. 2008) and forest matrices (Steinfartz et al. 2007). We believe that a more comprehensive conceptual framework can better explain the low rate of sympatric speciation.

So why is sympatric speciation rare? Is it merely because, as Mayr (1963) pointed out, of the difficulty of developing reproductive isolation? Or are there other evolutionary phenomena that can prevent speciation in the absence of a geographic barrier? The former is doubtful because reproductive isolation can evolve rapidly (Rice and Salt 1990; Hendry et al. 2000; Hendry 2001). However, populations can exhibit a wide range of resource polymorphisms that can be induced by genetics and/or environmental cues. These polymorphisms can influence the likelihood of speciation events. As a result, some investigators have proposed that we consider populations as existing along a continuum of reproductive isolation, with panmictic populations on one extreme end and complete reproductive isolation on the other (Hendry 2009; Hendry et al. 2009a). Viewing populations along this continuum organizes variation of a key parameter to speciation and helps account for the wide variation that can occur within species. However, it also ignores many of the other outcomes that could occur in sympatry (Rueffler et al. 2006).

We propose that we instead consider a continuum with two axes, reproductive isolation and degree of divergence in ecological traits. Using this model, we can map several types of polymorphisms and determine which types could potentially lead to speciation and which will generate within-species diversity but ultimately halt the process 
of speciation. Rueffler et al. (2006) began organizing various outcomes of disruptive selection, which included sexual dimorphism, phenotypic plasticity, resource polymorphisms, and speciation, but did not unify all the components into an overall framework.

Our review investigates the varied types of population diversity that can evolve and how they can halt, hinder, or promote the process of sympatric speciation. We define ecological diversification as any diversity that arises from ecological interactions, such as foraging, competition, predation, and parasitism. In this review we focus on dietary diversity in part for simplicity. In addition, dietary diversity both affects and is affected by competition, which has long been acknowledged as a diversifying force. Finally the effects of dietary diversification on speciation have received relatively little attention. The conceptual framework presented in this paper has several advantages. The model does not dismiss the possibility of sympatric speciation, but proposes a pathway along which it can proceed. In doing so, the model explains that sympatric speciation is rare because a variety of evolutionary outcomes can occur in sympatry, some of which can increase genetic and phenotypic variation in populations yet never lead to speciation. Finally, the model helps clarify the relationship between ecological factors and evolutionary diversification, providing guidance for future research into eco-evolutionary dynamics. 


\section{THE CONCEPTUAL MODEL}

Overview: We will first discuss the forms of intraspecific diversity that can arise in sympatric populations and how they can develop. We present the model on two axes (Fig 1), reproductive isolation and degree of divergence in an ecological trait or set of traits. Reproductive isolation has long been the defining feature of discrete species (Mayr 1942) and degree of divergence in ecological trait(s) increases the evolution of polymorphisms and subsequent speciation (Débarre 2012). The conceptual model has two extreme ends, a panmictic population in the lower left and two reproductively isolated populations in the upper right. We place the speciation box in the upper right because diverging populations must attain reproductive isolation to speciate and because without ecological divergence between sympatric species one of them will likely be driven extinct by competitive exclusion (Hardin 1960).

The initial steps towards divergence are the development of different resource acquisition patterns, which are known as individual specializations (IS), and the subsequent aggregation of these patterns within populations. Different kinds of polymorphisms can potentially develop from such aggregations. Polymorphisms that develop into sexual dimorphisms or that are caused by heterozygotic advantage will be angenic, unable to speciate along that ecologically diverging trait because of the lack of reproductive isolation and the loss of intraspecific competition and ecological

opportunity. Other kinds of resource polymorphisms can develop and speciate if certain obstacles are overcome. Obstacles that can hinder or halt the process of speciation in these potentially cladogenic polymorphisms include cycles of specialization and disruptive selection and the difficulty of developing assortative mating. Species can often 
get stuck in these phases indefinitely, which is represented by the model's circular arrows. In the below subsections explaining the conceptual model, we first describe the ways in which resource acquisition patterns can begin to aggregate and how discrete morphologies may develop from those patterns. We then discuss how these resource patterns and subsequent polymorphisms affect speciation.

Initial differentiation: individual specialization and the aggregation of patterns: The first step of sympatric speciation through resource specialization occurs when diverse patterns of resource use arise among individuals in a panmictic population and in the absence of any discrete morphotypes (Wennersten and Forsman 2012). These individual specializations (IS) were once believed to be extremely rare. However, Bolnick et al. (2003) found evidence that IS occurs in 93 species, and more recent studies have demonstrated IS in at least 18 more. Our developing knowledge of IS can enhance our understanding of ecological communities and their evolutionary potential because IS provides the variation from which discrete resource polymorphisms can evolve (Schluter 2001; Bolnick et al. 2003).

Individual specializations are driven by proximate causes but are also influenced by ecological conditions. The driving proximate mechanism behind IS is differences in prey preferences. Those differences in prey choice may arise from learning (Estes et al. 2003; Sargeant et al. 2005; Sargeant and Mann 2009), trade-offs between habitat and phenotype (Schluter and McPhail 1992; Schluter 1998; Matthews et al. 2010), or physiology (Afik and Karasov 1995). The ecological conditions that favor the development of IS are high 
levels of intraspecific competition and ecological opportunity (Nosil and Reimchen 2005; Svanbäck and Bolnick 2007; Martin and Pfennig 2010; Yoder et al. 2010); these ecological conditions can promote the development of similar patterns of resource use among individuals. Intraspecific competition causes individuals to seek out the alternative prey they are best at capturing (Svanbäck and Bolnick 2005; Tinker et al. 2008). Ecological opportunity is determined by the availability of resources that can be incorporated into the niche. When ecological opportunity is high, there are many alternative prey types available if intraspecific competition increases. Ecological opportunity increases with ecological release (Yoder et al. 2010), which may occur by the extinction of a predator or interspecific competitor (MacArthur et al. 1972; Losos 2010), by some mutation (Losos 2010), or by colonization of a new area (Losos 2010), especially islands (for terrestrial animals) and lakes (for aquatic species), where there are relatively depauperate assemblages of predators or competitors. The recent radiation of fish in newly formed northern lakes is thought to have occurred because rare colonization events offered the colonizers ample ecological opportunity (Schluter 1998, 2001). The presence of parallel benthic and limnetic morphs in species inhabiting different lakes lends support to this idea (Nosil 2012).

If the traits associated with IS and other resource specializations are heritable or environmentally induced, then various morphotypes may develop. The type of polymorphism that develops will depend upon the genetics of the distinct morphologies and the environment where the morphotypes are found (West-Eberhard 2003; Bolnick and Doebeli 2003; van Dooren 2006). If the trait in question depends upon gender, then a sexual dimorphism can develop (Bolnick and Doebeli 2003). If the polymorphism is 
caused by heterozygotic advantage and overdominance, than a heterozygotic polymorphism will form (van Dooren 2006). If the trait behind a given IS is not dependent on gender and heterozygotes do not have any advantage over homozygotes, than cladogenic polymorphisms may develop.

Sexual dimorphism: Morphological differences between the sexes have intrigued evolutionary biologists since Darwin and Wallace (Darwin 1871; Wallace 1889). Although sexual dimorphism is generally attributed to sexual selection, Darwin mentioned that differences between the sexes could also result from ecological conditions (Darwin 1859). Furthermore, sexual differences in traits related to foraging or digestive structures have long been considered a mechanism for intraspecific niche partitioning (Selander 1966; Schoener 1967; Shine 1989; Pérez-Barbería et al. 2008). We have included sexual dimorphism in our conceptual model of sympatric speciation because ecological differences between the sexes can have evolutionary consequences. Both sexual and natural selection can drive the evolution of differences in body size and feeding apparatus size/shape simultaneously. Even when differences are driven primarily by sexual selection, as occurs for example in body size dimorphism in many primates and pennipeds, those differences can have secondary ecological effects, which have ramifications for the evolutionary potential of the species in which they occur.

Theoretical work by Slatkin (1984) demonstrated that intraspecific competition was the primary driver in the development of sexual dimorphism from ecological factors. Recent work corroborated this finding and showed that when traits associated with resource acquisition depend upon gender, sexual dimorphism evolves (Bolnick and Doebeli 2003). When the traits are independent of gender, however, then the resource 
acquisition patterns can develop into resource polymorphisms that can potentially speciate (Bolnick and Doebeli 2003). These divergent fates are strikingly illustrated by cichlid species that differ in the genetics of color determination. In a cichlid species pair in Lake Malawi, coloration is sex dependent (Barson et al. 2007) and a sexual dimorphism in color has arisen. In contrast, in the Central American genus Amphilophus gold and dark color morphs are not sex dependent (Geiger et al. 2010; Fan et al. 2012). The latter cichlids may have already developed reproductive isolation between the color morphs, and some authors have suggested that sympatric speciation has occurred (Barluenga et al. 2006; Barluenga and Meyer 2010). In contrast, the Lake Malawi cichlid species cannot develop reproductive isolation because the sexes will always choose mates of the opposite color.

Despite the theoretical arguments for the ecological origins of sexual dimorphisms (Slatkin 1984; Bolnick and Doebeli 2003), there is so far only one strong example in which ecological pressures have driven the development of a sexual dimorphism: the differentiation of bill curvature in purple-throated carib hummingbirds, in which males' and females' bills are shaped for foraging optimally on different species of Heliconia flowers (Temeles et al. 2000). The paucity of empirical evidence that clearly demonstrates this phenomenon is due to the difficulty of identifying purely ecological vs. purely sexual traits.

The original criterion used to determine whether a trait evolved through sexual or natural selection was whether it was involved in feeding or courtship (Selander 1966). But separating traits on this basis may not capture the entire picture of how sexual selection acts on phenotypes because some traits may be selected for by both sexual and 
natural selection based on ecological factors. For example, it may be more advantageous for females of some mammalian species to have a more nutritious diet not only because they have a smaller gut but also because they need the extra nutrients for gestation (Pérez-Barería et al. 2008). Similarly, female snakes may have relatively larger heads than males because that sexual difference in head size either reduces competition for food between the sexes (Shine 1991) or increases female fecundity. Finally, Temeles (1985) showed that both natural and sexual selection may be acting to increase body size dimorphism in bird-eating raptors. Since males and females have different body sizes, a breeding pair can effectively exploit both larger and smaller prey. Thus having two differently sized hawks supporting a nest increases the amount of food brought to that nest, increasing the offspring survival (Temeles 1985).

Traits may originate through sexual selection but then be reinforced through ecological factors (and although we have as yet no examples, the reverse could also be true). In a study on emydid turtles, Stephens and Wiens (2009) demonstrated that most of the sexual size dimorphism in this group was caused by increased fecundity for larger females. But within the Graptemys-Malaclemys clade, there appeared to be increased sexual size dimorphism due to ecological factors, a secondary ecological reinforcement of the existing dimorphism (Stephens and Wiens 2009). A second example of secondary ecological reinforcement is in the northern elephant seal. Sexual selection has undoubtedly acted in the evolution of sexual size dimorphism in this species, but the large size differences between the sexes renders them different ecological species, with different diets, behaviors, and predation risks (Staniland 2005). Ecological consequences of sexual dimorphisms in traits that may be related to diet (as well as other ecological 
parameters such predation and parasitism) must be taken into account to understand the evolutionary history and potential of a species.

Protected polymorphisms: migration-selection and heterozygotic advantage: Levene (1953) began a promising theoretical inquiry into the maintenance of polymorphisms, a line of inquiry that would lead to the definition and discussion of protected polymorphisms. Two types of protected polymorphisms, migration-selection and heterozygotic advantage, are defined based on the mechanisms that promote them, and these mechanisms affect whether the polymorphisms can develop in sympatry. In heterogeneous environments, polymorphisms can be maintained through a particular migration-selection balance (Ravigné et al. 2009). In the models that predict polymorphisms, migration between environments is low and selection is strong (Christiansen 1974; Karlin and Campbell 1981; Bürger 2009). This type of protected polymorphism is also referred to as local adaptation, whereby populations adapt to local environments. Since these models predict that polymorphisms are maintained by differential adaptation to varying habitats, the issue of whether this is occurring within a sympatric population needs to be raised.

Authors have differed in their definitions of sympatry, resulting in confusion about whether certain speciation events actually occurred in sympatry. To reduce that confusion, Fitzpatrick et al. (2008) reviewed the published definitions and organized them into two groups, biogeographic and genetic. All definitions shared either a high degree of range overlap (biogeographic) or a high migration rate (genetic). The genetic 
definitions are largely heuristic. They assume speciation began when $\mathrm{m}=0.5$, i.e. when migration was free, but it is impossible to determine if populations were originally freely migrating once polymorphisms have developed any reproductive isolation (Mallet et al. 2009). We therefore use a biogeographic definition, in which individuals of diversifying populations occupy the same space at the same time and frequently encounter one another (Fitzpatrick et al. 2008). Given this definition, since migration must be high in order for a population to be considered sympatric, migration-selection models are unlikely to be relevant to sympatric speciation.

Models that produce polymorphisms from heterozygotic advantage differ in several ways from models that predict protected polymorphisms from migration-selection (Table 1), although both require strong trade-offs (van Dooren 2006; Ravigné et al. 2009). Polymorphisms protected by heterozygotic advantage can evolve in panmictic populations under conditions of heterosis and strong overdominance (Prout 1968; van Dooren 2006). In constrast, polymorphisms protected by a migration-selection balance have low migration/dispersal (Levene 1953) and low to intermediate overdominance (Bürger 2009). Polymorphisms maintained by migration-selection can potentially be diversifying if one population develops reproductive isolation via selection or drift. This is the type of situation that is at the very heart of many forms of allopatric speciation. Heterozygotic polymorphisms, on the other hand, have some protection from speciating and from disappearing. They are angenic because they require disassortative mating, which mixes genes among the polymorphisms. 
Sympatrically Cladogenic Polymorphisms: Sympatrically cladogenic polymorphisms differ from protected polymorphisms in that no heterozygotic advantage or overdominance occurs and that they can develop beyond morphotypes to speciate in sympatry. Sympatrically cladogenic polymorphisms can develop either via a genetic route that starts with a mutation or through developmental plasticity.

Most theoretical considerations of sympatric speciation focus on the genetic model (Seger 1985; Dieckmann and Doebeli 1999), which therefore needs relatively little explanation. In this model, a mutation produces a novel phenotype that allows an individual to specialize successfully upon some underutilized resource and avoid competition from its more abundant counterparts. The high fitness of individuals with the mutation will result in its rapid spread through the population. This substitution rate, however, is generally expected to be relatively slow, which is one explanation as to why speciation is not a quick process. Disruptive selection drives intermediate phenotypes between the old and new morphs extinct, at which point assortative mating can maintain distinct genetic lineages that can develop strong mechanisms for reproductive isolation. This model has widely been accepted since the modern synthesis of evolution, but has recently been challenged by an alternative model in which plastic traits can drive some of the initial steps of speciation.

Phenotypic plasticity has generally become accepted as a strong evolutionary force (Price et al. 2003; West-Eberhard 2003; Pfennig et al. 2010; however, see Thibert-Plante and Hendry 2011a). It can result in novel phenotypes that can utilize a previously unused resource, thereby initiating niche expansion and forming resource polymorphisms that can then diverge (West-Eberhard 2003; Pfennig et al. 2010). However, plasticity will 
shield the polymorphism from any further divergence unless the traits come under genetic control and can be inherited. Plastic traits can come under genetic control via a two-step process, genetic accommodation and genetic assimilation (Schlichting 2004; WestEberhard 2003).

When a population encounters a new resource, a plastic response can enable an entire subset of the population to begin using it as part of its niche. This plasticity could potentially overcome the problem of slow substitution rates (Price et al. 2003; Ghalambor et al. 2007), but for this type of polymorphism to proceed toward speciation, the environmentally determined plastic trait must undergo genetic accommodation, becoming refined by genetics to allow optimal adaptation (West-Eberhard 2003). If the environment remains stable, plasticity is no longer advantageous and the trait begins to be genetically determined (DeWitt et al. 1998). Once genetic assimilation has occurred, the process toward speciation can proceed along the same path as in the genetic route, where disruptive selection favors the extreme traits and the intermediate forms go extinct.

The costs of plasticity determine whether a trait will undergo genetic accommodation and assimilation. They can result from maintenance or production of the plastic trait and from information acquisition (DeWitt et al. 1998). When these costs are lower than the benefits of plasticity over time, assimilation is more likely to occur. Plasticity is likely to be more beneficial in fluctuating or locally variable environments (Svanbäck et al. 2009), suggesting that stable environments may be more conducive to speciation (Knudsen et al. 2010). 
Clearly, empirical evidence supports the idea that polymorphisms can arise through either mutation or developmental plasticity (Table 2). Current literature (Table 2) suggests that the two routes are equally frequent over all taxa, and accordingly, neither should be ignored if we are to build a complete picture of sympatric speciation. Phenotypic plasticity appears to be much more common in fishes and amphibians. Many of the well-studied lacustrine polymorphisms and species complexes in fishes of the Northern Hemisphere are driven by phenotypic plasticity (three-spined sticklebacks: Wund et al. 2012; sunfishes: Ellerby and Gerry 2011, Parsons and Robinson 2006; Arctic char: Adams and Huntingford 2004; Eurasian perch: Svanbäck and Eklöv 2006; and Midas cichlids: Muschick et al. 2011). Within amphibians, clades with more plasticity have more species and utilize more habitats (Pfennig and McGee 2010), and parallel evolution consistent with the phenotypic plasticity model has occurred within several anuran taxa (Scaphiopodidae, Pelobatidae, and Pelodytidae: Pfennig and McGee 2010). Furthermore, proportions of cannibalistic morphs of spade-foot toads (Martin and Pfennig 2010) and salamanders (Collins and Cheek 1983; Semlitsch et al. 1990) are driven by changes in their aquatic environments.

Although phenotypic plasticity can also drive polymorphisms in birds (van de Pol et al. 2009) and mammals (Wolf et al. 2008; Table 2), resource polymorphisms in mammals, birds, and invertebrates appear to originate more often from genetic mutations. For example, in mammals, there are genetically distinct morphs of North Atlantic killer whales, Orcinus orca, (Foote et al. 2009) the domestic mouse, Mus musculus domesticus in (Hauffe and Searle 1993; Piálek et al. 2001). In birds, there are genetically distinct morphotypes in the African finch, Pyrenestes ostrinus (Smith 1993), and the medium 
ground finch, Geospize fortis (Huber et al. 2007), the latter of which has developed some reproductive isolation (Huber et al. 2007). A marine snail, Littorina saxatilis, developed from genetically distinct morphs (Conde-Padín et al. 2007) as well as several species of dipterans (Feder et al. 2003; Diegisser et al. 2007; Simard et al. 2009)

While the literature in Table 2 illustrates how sympatrically cladogenic polymorphisms develop, it does not demonstrate which mode of development more quickly leads to speciation. As a result of several factors, the dynamics of the transition from resource polymorphisms to daughter species will largely be system-dependent, with some genetic systems moving faster than some plastic systems and vice versa. The tempo of speciation through the genetic model depends on how the substitution rate is affected by trait dominance, recombination rates, and relationships among traits. Dominant traits will have higher substitution rates, but high recombination rates will retard the development of genetic lineages (Hey and Kliman 2002; Connallon and Knowles 2007). Linkage between traits can slow speciation if the linked gene is detrimental to the new morph or speed the process if the linked gene is beneficial (Streisfeld and Rausher 2010). Epistasis and pleiotropy have similar effects on the substitution rate, slowing it when the relationships are detrimental and speeding it when beneficial (Hawthorne and Via 2001, Østman et al. 2012). Furthermore, population size may also affect how quickly one allele can replace another because of genetic drift at small numbers of individuals (Andolfatto 2007).

Many proponents of the developmental model suggest it is faster because of its initial speed, when individuals can immediately incorporate a new resource (West-Eberhard 2003, Moczek et al. 2011). However, the plastic traits must become genetically controlled 
and thereby heritable before speciation can occur. In addition, assimilation may be subject to substitution rates similar to those for any allele, thus slowing speciation through the developmental route to the same rate as through the genetic route, at least for the step of assimilation, and subjecting the process to the effects of the genetic factors discussed above. Finally, genetic assimilation is unlikely to happen simultaneously across a population. Instead, it may happen piecemeal, and genetic assimilation itself may have a substitution rate.

\section{SPECIATION TRAPS}

A speciation trap is a polymorphism that increases intraspecific diversity while preventing speciation based on the trait(s) in question. In our model, the speciation traps are sexual dimorphisms and heterozygotic polymorphisms. Both types of speciation traps halt progress towards speciation by reducing intraspecific competition and decreasing ecological opportunity, and heterozygotic polymorphisms also discourage assortative mating. Each type of polymorphism is evolutionarily stable and is only a "trap" in the sense that it prevents any diversification from attaining reproductive isolation. In this section we look at how each type of speciation trap prevents the development of reproductive isolation.

Evolutionary ramifications of sexual dimorphisms: Sexual dimorphism can hinder speciation whenever differences between sexes act to separate their niches. There are three reasons for this. First, opposing directional selection between the sexes (Fig. 2) 
results in the evolution of sexual dimorphisms that will reduce potentially diversifying competition. Closely related sympatric species show greater sexual size dimorphism than do closely related allopatric species (Stephens and Wiens 2009), suggesting that the evolution of sexual size dimorphism is an alternative way to reduce competition and thus may inhibit speciation (Bolnick and Doebeli 2003; Stephens and Wiens 2009). Previous work has also demonstrated that populations with the highest sexual dimorphism exhibit the least disruptive selection (Bolnick and Lau 2008), similarly suggesting that these populations are not diversifying in a way that will allow speciation. Second, the two sexes' occupation of different subniches decreases ecological opportunity. This can either prevent a group of conspecifics from occupying that now-used resource space or (from an allopatric standpoint) prevent a different species from another area from invading the resource space and differentiating from its source population into a new species. Third, sexual dimorphism will lead to disassortative mating, which prevents reproductive isolation.

Niche separation between the sexes appears to be common in sexually dimorphic species. In all of the 81 species listed in Table 3, which are from eight taxonomic groups, the two sexes occupy slightly different niches. This non-exhaustive list is strongly biased toward vertebrates, and specifically toward birds and mammals, which may be an artifact of research interests rather than a taxonomic bias in the occurrence of the phenomenon. Differences in body size are responsible for niche differentiation in most of these species. In primates (Kamilar and Pokempner 2008) and ungulates (du Toit 2005; Pérez-Barbería et al. 2008), for example, males are often larger, consequently have larger guts, and can therefore subsist on lower quality food (Pérez-Barbería et al. 2008). Size differences in 
some sea birds (Bearhop et al. 2006; Phillips et al. 2009) and seals (Staniland 2005;

Staniland and Robinson 2008) allow the larger sex to dive deeper and forage farther from shore. Niche differences can also be related to the size and shape of feeding structures, such as beaks in birds (woodpeckers: Kilham 1965, Selander 1966; hummingbirds:

Temeles et al. 2000) and mouthparts in invertebrates (Clark 1963; Whiting 1967; Atchley and Martin 1971). Researchers have inferred that morphological differences between the sexes cause diet differentiation in numerous other species, including 50 snakes (Shine et al. 1991), seven anole lizards (Butler et al. 2000), and 17 hummingbirds (Temeles et al. 2010); these species were not included in Table 3 because the relationship between diet and sex was not directly tested.

Opposites attract: heterozygotic advantage and disassortative mating: Protected resource polymorphisms that occur in sympatry result from heterozygotic advantage and overdominance (van Dooren 2006), which can prevent the polymorphism from continuing toward speciation for two reasons. First, in such polymorphisms, the heterozygote will by definition have a selective advantage, and dissortative mating is accordingly favored for homozygotes. Homozygotes will always have a lower fitness than heterozygotes but will never go extinct because they are a by-product of heterozygotic mating. Thus the resource polymorphism is maintained, but the selectively advantageous disassortative mating can prevent the development of reproductive isolation, and thus halt speciation. Second, the phenotypic differences among polymorphs reduce intraspecific competition (Bolnick et al. 2003; Svanbäck and Bolnick 2007; 
Martin and Pfennig 2009, 2010; Bernard and Maher 2011). This reduction of intraspecific competition relieves selective pressures that would otherwise promote speciation.

Evidence of protected polymorphisms maintained by heterozygotic advantage is rare. Most empirical work on heterozygotic advantage has shown that positive associations between fitness and heterozygosity are weak at best (David 1998; Ding and Goudet 2005). Most studies that do find a strong selective advantage for heterozygosity involve genes in the major histocompatibility complex and other host-parasite interactions (Penn et al. 2002). However, the snail Cepaea nemoralis, which is polymorphic in shell color and banding, does appear to be a strong candidate for the heterozygotic advantage model (Cook 2007). Although frequency-dependent disruptive selection from predation was once thought to drive this polymorphism (Clarke 1969), more recent work has shown that predation does not appear to be a strong selecting force on this species and that the dominant phenotypes are heterozygotic (Cook 2007). More work investigating the frequency of heterozygotic advantage is required before we can satisfactorily determine its importance as a block to sympatric speciation.

\section{SPECIATION OBSTACLES}

In contrast to speciation traps, speciation obstacles do not intrinsically prevent reproductive isolation but will slow down the process of speciation or keep a population at a stage of specialization or discrete morphotypes indefinitely. The first obstacle is the presence of individual specialization, which can promote species coexistence and result in the loss of ecological opportunity. Two other obstacles, cycles of specialization and 
cycles of disruptive selection, are related to changing ecological conditions that alter selection pressures. The fourth obstacle is the difficulty of developing assortative mating.

Specialization, coexistence, and the loss of ecological opportunity: Individual specializations (IS) can decrease the interaction strength among competing species (Lichstein et al. 2007; Bolnick et al. 2011; Lasky et al. 2014). When individuals use a smaller subset of the population's dietary resources, the number of individuals from each species that overlap in resource use will be reduced (Bolnick et al. 2011; Lasky et al. 2014). As a result, the resources may not become limiting. Individual specializations can therefore promote species coexistence and increase the number of species present in a community (Lichstein et al. 2007; Lasky et al. 2014). The greater number of species in a community will decrease available ecological opportunity that could have been used for the niche expansion needed to develop more distinct polymorphisms. Therefore, the effects that IS has on species coexistence may disrupt to the conditions that are required for sympatric speciation. Indeed, many examples of ecologically driven resource polymorphisms occur in environments where there are not many heterospecific competitors (Smith and Skúlason 1996; Schluter 2002; Adams and Huntingford 2004; Knudsen et al. 2006; Costa et al. 2009).

Cycles of specialization: Different ecological conditions favor individual specialists versus individual generalists (Fig. 3). For example, intraspecific competition increases individual specialization (IS; Svanbäck and Bolnick 2007; Martin and Pfennig 2009, 
Svanbäck et al. 2011; Agashe and Bolnick 2010), while interspecific competition decreases it (Darimont et al. 2009). Anecdotal evidence also suggests that increased resource diversity increases IS (Herrara et al. 2008; Matich et al. 2011). Finally, high predation levels decrease IS because increased predation tends to limit the number of habitats a prey organism may visit (Araújo et al. 2011).

Although little empirical research directly tests the idea that populations can switch between individual specialists and individual generalists, there are four strong examples of this phenomenon in vertebrates. One study that focused on the effects of resource diversity on IS found that Egyptian fruit bats (Herrera et al. 2008) showed the highest degree of specialization in spring, when the greatest number of plant species fruit. The other three studies demonstrated that individuals specialized when intraspecific competition was high. For example, individual perch, Perca fluviatilis, specialized more when intraspecific density was high (Svanbäck and Persson 2004), and similarly, sea otters (Tinker et al. 2008) specialized when food was limited but were generalists when it was not. In the only experimental work to test whether differences in intraspecific competition affect IS, Svanbäck and Bolnick (2007) replicated naturally occurring densities of threespined stickleback in enclosures and found that the high levels of intraspecific competition at high densities increased IS.

It is well known that ecological pressures are not constant over time: resource abundances and levels of competition and predation fluctuate. Such natural fluctuations might prevent specializations from becoming established as polymorphisms, resulting in no further evolutionary diversification. Accordingly, IS can become as much a hindrance to the process of speciation as a starting point for it. 
Cycles of disruptive selection: Disruptive selection is an integral part of the development of new species in sympatry, for it is the evolutionary force that can split a population into an adaptive landscape with two fitness peaks (Maynard-Smith 1966; Seger 1985; Dieckmann and Doebeli 1999). Disruptive selection was long thought to be rare and as a result inconsequential, but recent work has demonstrated that it is more common than previously thought (Smith 1993; Bolnick 2004b; Calsbeek and Smith 2007; Quesada et al. 2007; Bolnick and Lau 2008; Martin and Pfennig 2009; Hendry et al. 2009b). This recent research has also shown that disruptive selection is inconsistent and, by itself, cannot result in speciation (Nosil et al. 2009). Populations can become stuck in the disruptive selection phase either due to inconsistent ecological pressures or through lack of assortative mating (discussed in the following section).

A great deal of research has examined frequency-dependent disruptive selection, but relatively few studies have considered its long-term effects on population dynamics. Cycles of disruptive selection can be caused by changes in the relative frequency of conspecific variants (Hori 1993; Benkman 1996; Bolnick et al. 2004b; Martin and Pfennig 2009). Strong, frequency-dependent, intraspecific competition initiates disruptive selection (Seger 1985; Dieckmann and Doebeli 1999; Doebeli et al. 2007) in which rare, extreme phenotypes experience less competition than more common, intermediate phenotypes (Seger 1985; Martin and Pfennig 2009; Benard and Maher 2011). Because these common phenotypes are initially selected against, they will become rare while extreme phenotypes become more common. At this point, however, the intermediate phenotype has a selective advantage and selection becomes stabilizing (Lewontin 1958; 
Hori 1993; Bolnick 2004b). This can generate a stable polymorphism with fluctuating phenotypic frequencies that can inhibit speciation indefinitely. In a review of selection in natural populations, Kingsolver et al. (2001) found that disruptive and stabilizing selection were about equally common and equally strong, suggesting that it is possible for species to be subjected to both disruptive and stabilizing selection at different times.

Changes in other ecological factors such as climatic variation (Hairston and Dillon 1990; van de Pol et al. 2009) and resource distribution (Hendry et al. 2006; Quesada et al. 2007) can also drive changes in selection pressures. For example, a long-term study of a natural population of oystercatchers showed that selection is stabilizing and favors generalist individuals in most years (van de Pol et al. 2009). In particularly harsh winters, however, selection becomes disruptive and favors specialist individuals. Similarly, anthropogenic changes in resource distributions can alter the fitness landscape. For example, beak size of Darwin's Finches on Santa Cruz Island shifted from a bimodal to a unimodal distribution (Hendry et al. 2006) where humans had modified their resources but remained bimodal in an unaltered area on the same island (Hendry et al. 2006).

Indefinite disruptive selection: the special problem of assortative mating: A lack of assortative mating was recognized by Maynard-Smith (1966) as being one of the most serious obstacles to sympatric speciation because it reduces the genetic differences between morphs. For example, disruptive selection has been persistent in African Pyrenestes finches, but because little to no assortative mating occurs, that selection will not lead to speciation until some mechanism of assortative mating evolves (Smith 1993). 
Greater adaptive divergence and speciation are similarly prevented in sympatric lakestream species pairs of three-spined sticklebacks (Hendry et al. 2002), and even a small amount of gene flow across races has prevented speciation in many host races of phytophageous insects (Drès and Mallet 2002; Prowell et al. 2004).

Assortative mating can be costly, preventing the development of reproductive isolation. One common detriment of assortative mating is that the requisite choosiness in mates may result in a failure to mate (Bolnick 2004a; Kopp and Hermisson 2008). However, a theoretical model by Kopp and Hermisson (2008) showed that choosiness was a substantial cost only when females had fewer than ten mating opportunities. In general, the fitness costs of assortative mating outweigh any selective advantages associated with it (de Cara et al. 2008). However, in a review Jiang et al. (2013) found that assortative mating was not uncommon in nature, but was generally weak.

The barrier of assortative mating can be overcome if certain conditions are met. In one-trait models, in which the ecological and assortative mating traits are pleiotropic, the development of assortative mating and reproductive isolation is favored because ecologically driven disruptive selection is by definition associated with assortative mating (Kirkpatrick and Ravigné 2002; Gavrilets and Vose 2005; de Cara et al. 2008). In twotrait models, the development of assortative mating is less likely but can occur if the linkage disequilibirum for the ecological and mating preference traits is high enough to overcome recombination (Kirkpatrick and Ravigné 2002; de Cara et al. 2008). Finally, assortative mating is more likely to develop when homozygotes are more fit than heterozygotes (Otto et al. 2008). 
In sum, disruptive selection can act as a hindrance to speciation or promote it. The factors that determine disruptive selection, intraspecific competition and resource diversity, can change over time and cause changes in the occurrence and strength of selection. Populations can thus be prevented from speciation by cycles of disruptive and stabilizing selection, as well as by weak assortative mating.

\section{SPECIATION}

What it takes to speciate: Moving beyond polymorphisms to reach reproductive isolation and thus complete the process of speciation depends upon two intertwined factors: the nature of selection and the strength of assortative mating. There is currently no definitive answer as to whether speciation is more likely when strong selection acts on a single or few traits (Maynard-Smith 1966; Schluter 2001; Rundle and Nosil 2005), or when selection is weaker but multifarious (Rice and Hostert 1993; Via 2001; Dambroski and Feder 2007). Strong selection alone does not appear to be sufficient to cause speciation because it is unlikely to be associated with the evolution of assortative mating and thus may merely entrench a single-trait polymorphism (Nosil et al. 2009). Multifarious selection (Rice and Hostert 1993; Via 2001; Dambroski and Feder 2007) may seem inherently more likely to lead to reproductive isolation because of the increased probability of some form of genetic incompatibility between morphs due to selection on multiple traits. But multifarious selection is often weak and therefore unlikely to cause reproductive isolation in the face of high gene flow (Nosil et al. 2009). 
Researchers have used genomics in attempts to elucidate whether strong or multifarious selection better stimulates speciation (reviewed by Elmer and Meyer 2011), with inconclusive results. Kuatt et al. (2012) showed that strong selection has occurred on a small genomic region in two species of the Midas cichlid group (Amphilophus citrinellus and A. labiatus). However, Kuatt et al. (2012) also found that despite considerable morphologic differentiation between these two named species, there is also considerable gene flow between them, indicating that they might not be fully speciated as previously thought. In contrast, genomic work on two distinct morphs of three-spined sticklebacks in Paxton Lake suggested that weak, multifarious selection may be driving their divergence (Arnegard et al. 2014), although speciation is incomplete in this system.

The likelihood of speciation depends on the degree of relatedness between the genetic traits that determine ecological divergence and reproductive isolation (Hawthorne and Via 2001; Rundle and Nosil 2005). Speciation is more likely to occur when there are fewer genes associated with the relationship between the traits causing divergence and the traits causing reproductive isolation (Gavrilets and Vose 2005; de Cara et al. 2008; Smadja and Butlin 2011). This was long ago acknowledged by Maynard-Smith (1966), who declared that one-allele models are more likely to predict speciation than two-allele models. The single traits of one-trait models are also referred to as magic traits (Servedio et al. 2011; Thibert-Plante and Gavrilets 2013) because these traits lend themselves to sympatric speciation. For example, phytophagous insects mate on the plants on which they feed, a magic trait that can result in host shifts which would promote speciation (Emelianov et al. 2001). This characteristic makes phytophagous insects appealing for research on sympatric speciation. 
When two or more traits are involved between the ecological and assortative mating traits, then recombination can prevent the build-up of linkage. In order to overcome this problem, either the linkage must be originally very tight or recombination must be suppressed (Trickett and Butlin 1994). The most common way to suppress recombination is through chromosomal rearrangements that interfere with meiotic drive, making hybrids sterile or less fit (Trickett and Butlin 1994; Noor et al. 2001; Rieseberg 2001). Such chromosomal rearrangements have been demonstrated in Drosophila (Coyne et al. 1993; Navarro and Ruiz 1997; Álvarez-Castro and Álvarez 2005), mice (Hauffe and Searle 1993; Piálek et al. 2001), the mosquito Anopheles gambiae (Simard et al. 2009), and three-spined stickleback (Jones et al. 2012), and they may have contributed to speciation in the Midas cichlid complex (Kuatt et al. 2012). Further work is required to demonstrate any generality of this mechanism in promoting the completion of speciation.

One potential way to link the traits of ecological divergence and reproductive isolation is through sexual selection on ecologically divergent traits (Schluter 2001, 2002; Maan and Seehausen 2001). This can happen through selection on secondary sexual traits (Lande 1982), differential mate recognition (Ryan and Rand 1993), or differential use of mating habitat (Endler 1992). Although this process represents a potential route toward speciation, it still may present the same problems that arise whenever two or more alleles are involved if the traits under sexual and ecological selection are not determined by the same gene. Then, if polymorphisms are to proceed toward speciation, either the genes on which they are based must be tightly linked or recombination must be suppressed through chromosomal rearrangement. 
Stored diversity: The polymorphisms and individual specialization discussed in this paper result in a breadth of diversity that can develop within populations and species. This diversity can be stored in a population and act as standing genetic variation. The stored diversity can spread geographically if a group of individuals in the population colonize a new area and may make possible colonization of areas in which these individuals would not have been able to successfully establish without the development of intrapopulation niche variation in sympatry. In these conditions, diversifying processes in sympatry may facilitate speciation in allopatry.

Standing genetic variation, or stored diversity, contributes more to adaptation than a given new mutation for two reasons (Barret and Schluter 2007). First, adaptation can proceed faster from stored diversity because the alleles associated with it have a greater frequency of occurrence in the population, whereas adaptations from new mutations will require at least several generations to spread (Innan and Kim 2004). Second, stored diversity has already been subject to selection and any deleterious alleles will likely have been removed (Barret and Schluter 2007) increasing the chances that alleles associated with the stored diversity are advantageous.

There are several strong examples of adaptation occurring from standing variation, and they are taxonomically diverse. The first and probably best-known example is the apple maggot fly in the northeastern U.S.A., where standing variation in diapause behavior from Mexico has facilitated speciation (Feder et al. 2003; Michel et al. 2007). In this case, standing variation arrived from an allopatric region and was advantageous when Europeans brought apples, which fruited later than hawthorns and changed the adaptive landscape. Similarly, variation in bony lateral plates in three-spined sticklebacks 
aided adaptation to freshwater lakes colonized from oceanic habitats (Barret and Schluter 2007). Finally, variation in coat color of mainland populations of the mouse Peromycus polionotus facilitated the development of the light coat color that was adaptive on beaches in the Santa Rosa Island population (Hoesktra et al. 2006; Barret and Schluter 2007). The taxonomic breadth and strength of these examples suggest that standing variation is likely to be broadly important in adaptation and speciation.

Evolutionary and conservation biology offer opportunities to perform empirical research on how stored diversity can promote speciation in allopatric conditions. The ways in which invasive species adapt to new environments and native species adapt and shift ranges in response to climate change may illuminate the relationship between stored diversity and allopatric speciation. If individuals belonging to some adaptively distinct subset of a colonization group have relatively high survival rates, researchers can determine which traits increase their survival and how those traits interact with the environment to enhance those individuals' fitness. These special instances of adaptation and range expansion may provide researchers with the opportunity to design experiments that can capture the dynamics among colonization, the later evolution of invasive species, and the population and community dynamics of the source populations. Likewise, understanding which types of individuals may be more likely to survive along the leading edge of a range expansion may help managers if ever humans attempt to intervene in range shifts associated with climate change. 


\section{CONCLUSIONS}

Sympatric speciation is rare for several reasons. First, some types of diversification such as sexual dimorphism and protected polymorphisms can result in speciation traps. Second, individual specialization and potentially cladogenic polymorphisms can be subjected to inconsistent ecological pressures, resulting in an absence of speciation. Species can be stuck in cycles of IS and disruptive selection when the ecological factors that are driving the specialization or disruptive selection are not maintained over time. Finally, assortative mating may never develop. As a result of these difficulties, it is rare that populations traverse the continuum from panmixis to complete reproductive isolation. 


\section{TABLES}

Table 1: Table demonstrating the differences between the two types of protected polymorphisms in the literature.

\begin{tabular}{|c|c|c|}
\hline & $\begin{array}{c}\text { Migration-Selection Balance } \\
\text { (Christiansen 1974, } \\
\text { Ravigné } \text { et al. 2009) }\end{array}$ & $\begin{array}{c}\text { Heterozygote Advantage } \\
\text { (Prout 1968, } \\
\text { van Dooren 2005) }\end{array}$ \\
\hline Migration/ Dispersal & Low & High \\
\hline Over-Dominance & Intermediate \\
\hline Trade-Offs & Strong & Strong \\
\hline Geographical Overlap & Allopatric & Sympatry \\
\hline Diversifying & & No \\
\hline
\end{tabular}


Table 2: List of species in which resource polymorphism originated from either that is determined either genetically or environmentally (i.e. following the developmental route of speciation).

\begin{tabular}{|c|c|c|c|}
\hline Taxonomic & & & \\
\hline Group & Species & Route & Reference \\
\hline \multicolumn{4}{|l|}{ Arthopods } \\
\hline & Tephritis conura & Genetic & Diegisser et al. 2007 \\
\hline & Rhagoletis pomonella & Genetic & Feder et al. 2003 \\
\hline & Anopheles gambiae & Genetic & Simard et al. 2009 \\
\hline \multicolumn{4}{|l|}{ Gastropod } \\
\hline & Littorina saxatilis & Genetic & Conde-Padín et al. 2007 \\
\hline \multicolumn{4}{|l|}{ Fish } \\
\hline & Pundamilia spp. & Developmental & Magalhaes et al. 2009 \\
\hline & Gasterosteus aculeatus & Developmental & Wund et al. 2012 \\
\hline & Lepomis gibbosus & Developmental & Parsons and Robinson 2006 \\
\hline & Lepomis macrochirus & Developmental & Ellerby and Gerry 2011 \\
\hline & & & Adams and Huntingford 2004, \\
\hline & Salvelinus alpinus & Developmental & Andersson et al. 2005 \\
\hline & Perca fluviatilis & Developmental & Svanbäck and Eklöv 2006 \\
\hline & Perissodus microlepis & Genetic & Hori 1993 \\
\hline & Coregonus lavaretus & Genetic & Østbye et al. 2004 \\
\hline & Cichlasoma managuense & Developmental & Meyer 1987 \\
\hline & & & Muschick et al. 2011 \\
\hline & Amphilophus spp. & Developmental & \\
\hline
\end{tabular}


Amphibians

$\begin{array}{ccc}\text { Pelodytes punctatus } & \text { Developmental } & \text { Jourdan-Pineau et al. } 2012 \\ \text { Spea multiplicata } & \text { Developmental } & \text { Martin and Pfennig } 2010 \\ \text { Pseudacris regilla } & \text { Genetic } & \text { Morey } 1990 \\ \text { Ambystoma taloideum } & \text { Developmental } & \text { Semlitsch } \text { et al. } 1990 \\ \text { Ambystoma tigrinum } & \text { Developmental } & \text { Collins } \text { et al. } 1983\end{array}$

Aves

$\begin{array}{ccc}\text { Haematopus ostralegus } & & \\ \text { ostralegus } & \text { Developmental } & \text { van de Pol } \text { et al. } 2009 \\ \text { Pyrenestes spp } & \text { Genetic } & \text { Smith } 1993 \\ \text { Neospiza } \mathrm{spp} . & \text { Genetic } & \text { Ryan } \text { et al. } 2007 \\ \text { Egretta sacra } & \text { Genetic } & \text { Rohwer } 1990 \\ \text { Geospiza fortis } & \text { Genetic } & \text { Huber } 2007\end{array}$

Mammals

Zalophus wallebaeki
Orcinus orca
Ursus americanus
kermodei
Mus musculus
domesticus
Developmental
Genetic

Wolf et al. 2008

Foote et al. 2009

Genetic

Hedrick and Ritland 2011

Genetic

Hauffe and Searle 1993 
Table 3: Species that exhibit sexual dimorphic traits that affect the ecology between the sexes, even if that sexual dimorphism is caused by sexual selection.

\begin{tabular}{|c|c|c|c|}
\hline \multirow{3}{*}{$\begin{array}{c}\text { Taxonomic } \\
\text { Group }\end{array}$} & \multicolumn{3}{|c|}{ Sexually } \\
\hline & \multicolumn{3}{|c|}{ Dimorphic } \\
\hline & Species & Traits & Citations \\
\hline \multicolumn{4}{|l|}{ Aves } \\
\hline & Neopmorpha acutirostris & Bill length, & Potts 1885 , Buller 1888, \\
\hline & $($ Extinct $)$ & bill depth & Selander 1966 \\
\hline & Cinclocerthia ruficauda & Bill length & Selander 1966 \\
\hline & & Cranial & \\
\hline & Sitta pusilla & skeleton & Norris 1958 \\
\hline & & Bill length, & \\
\hline & Limnodromus scolopaces & wing size & Pitelka 1950 \\
\hline & Limnodromus griseus & Bill length & Pitelka 1950 \\
\hline & & Pelvic limb, & Rand 1952, \\
\hline & Aechmophorus occidentalis & bill length & Livezey and Storer 1992 \\
\hline & & Pelvic limb, & \\
\hline & Aechmophorus clarkii & bill length & Livezey and Storer 1992 \\
\hline & & Body size, & \\
\hline & Acciptier striatus & bill size & Storer 1952 \\
\hline & Accipiter gentilis & Body size & Högland 1964 \\
\hline & Falco peregrinus & Body size & Cade 1960 \\
\hline & Spheniscus magellanicus & Body size & Forero et al. 2002 \\
\hline & Pygoscelis papua & Body size & Bearhop et al. 2006 \\
\hline & Eudyptes chrysolophus & Body size & Bearhop et al. 2006 \\
\hline & Phalacrocorax verrucosus & Body size & Bearhop et al. 2006 \\
\hline
\end{tabular}


Forero et al. 2005,

Phillps et al. 2011,

Phalacrocorax georgianus

Macronectes carbo sinensi

Macronectes halli

Rynchops niger intercedens

Diomedea exulans

Thalassarche chrysostoma

Phoebastria irrorata

Calonectris diomedea

Calonectris edwardsii

Sula sula

Sula leucogaster

Sula nebouxii

Centurus striatus

Dnedrocopos villosus

Archilochus colubris

Eulampis jugularis

Sephanoides sephanoides
Body size

Body size

Body size

Body size

Body size

Body size

Body size

Body size

Body size

Body size

Body size

Body size

Bill length,

bill depth

Bill length,

foraging strategy

Bill length,

bill shape

Bill curvature

Wing length,

bill size
Phillips et al. 2009,

Liordes and Goutner

2009

Phillips et al. 2009

Mariano-Jelicich et al.

2008

Weimerskirch et al. 1993,

Phillips et al. 2009

Phillips et al. 2009

Awkermen et al. 2007

Ramos et al. 2009

Ramos et al. 2009

Cherel et al. 2008

Young et al. 2010

Zavalaga et al. 2007

Selander 1966

Kilham 1965, Selander

1966

Berns and Adams 2010

Temeles et al. 2000,

Temeles et al. 2009

González-Gomez and

Estades 2009 
Bill and

\begin{tabular}{|c|c|c|}
\hline Limosa lapponia & leg length & Smith and Evans 1973 \\
\hline Picoides pubescens & Bill length & Peters and Grubb 1983 \\
\hline \multirow[t]{2}{*}{ Quelea quelea } & Bill length & Ward 1965 \\
\hline & Tail length, & \\
\hline Arses telescopthalmus & bill width & Bell 1982 \\
\hline Vireo olivaceus & Bill size & Williamson 1971 \\
\hline \multirow[t]{2}{*}{ Corvus corone } & Bill length & Holyoak 1970 \\
\hline & Bill and & \\
\hline \multirow[t]{2}{*}{ Cormobates leucopaea } & tongue length & Noske 1986 \\
\hline & Bill and & \\
\hline \multirow[t]{2}{*}{ Climacteris erythrops } & tongue length & Noske 1986 \\
\hline & Bill and & \\
\hline Daphoenositta chrysoptera & tongue length & Noske 1986 \\
\hline \multirow[t]{2}{*}{ Anolis conspersus } & Gape width & Schoener 1967 \\
\hline & Body size, & \\
\hline \multirow[t]{2}{*}{ Anolis polylepis } & gape width & Perry 1996 \\
\hline & Head length, & \\
\hline Morelia spilota imbricata & head depth & Pearson et al. 2002 \\
\hline
\end{tabular}

Mammalia

$\begin{array}{ccc} & \text { Condylobasel } & \\ \text { length, } & \\ \text { Herpestes ichneumon } & \text { canine diameter } & \text { Rosalino et al. } 2009 \\ \text { Vulpes vulpes } & \text { Carnassials } & \text { Viranta and Kauhala }\end{array}$


Mustela vison

Ovis canadensis

Cervus nippon

Giraffa camelopardalis

Tragelaphus strepsiceros

Rangifer tarandus

Cebus capucinus

Alouatta pigra

Cercopithecus campbelli

Cercopithecus mitis

Cercopithecus petaurista

Cercopithecus pogonias

Erythrocebus patas

Gorilla gorilla

Papio cynocephalus
Body size

Body size

Body size

Body size

Body size and

neck angle

Body size and

neck angle

Body size

Body size

Body size

Body size

Body size

Body size

Body size

Body size

Body size

Body size
Thom et al. 2004

Pérez-Barbería et al.

2008

du Toit 2005

du Toit 2005

du Toit 2005

du Toit 2005

du Toit 2005

Rose 1994

Kamilar and

Pokempner 2008

Kamilar and

Pokempner 2008

Kamilar and

Pokempner 2008

Kamilar and

Pokempner 2008

Kamilar and

Pokempner 2008

Kamilar and

Pokempner 2008

Kamilar and

Pokempner 2008

Kamilar and 
Pokempner 2008

Kamilar and

Pongo pygmaeus

Theropithecus gelada

Halichoerus grypus

Arctocephalus gazella

Arctocephalus forsteri

Arctocephalus pusillus

doriferus

Arctocephalus philippii

Cystophora cristata

Mirounga angustirostris

Mirounga leonina

Eumetopias jubatus

Otaria byronia

Odobenus rosmarus

Zodarion jozefienae
Body size

Body size

Body size

Body size

Body size

Body size

Body size

Body size

Body size

Body size

Body size

Body size

Body size
Pokempner 2008

Kamilar and

Pokempner 2008

Staniland 2005,

Tucker et al. 2007 ,

Lesage et al. 2001

Staniland and

Robinson 2008

Page et al. 2005

Page et al. 2005

Acuna and Francis

1995

Lesage et al. 2001,

Tucker et al. 2009

Le Boeuf et al. 2000,

Staniland 2005

Staniland 2005

Staniland 2005

Campagna et al. 2001

King 1983

Arachinda 


\section{Gastropoda}

Tricola Teeth of radulae

Echiura
Proboscis size

Size of

Phymatidae

Chironomidae

Braconidae

Mormoniella vitropennis

Pterygota

Ostracoda raptorial leg

Size of

labial teeth

Mouthpart

Mouthpart

Development

of maxillae
Robertson 1971

Gould-Somero 1975

Atchley and Martin 1971

Clark 1963

Whiting 1967

Kornicker 1985 


\section{FIGURE LEGEND}

Figure 1-The conceptual model displaying the various potential outcomes of sympatric animal populations laid out on two axes that both relate to traits, one ecological and the other to reproductive isolation. In addition to a pathway of ecological speciation, a sexual selection pathway and ways it can interact with ecological speciation are also presented with dashed lines

Figure 2-Diagram showing how sexual dimorphic traits can create sexually dependent directional selection and separate niche space between the sexes. a) Male and female niche dimensions before sexual dimorphism occurs. b) Uni-sexual directional selection, in this case males, relieving competition between the sexes. c) Di-sexual directional, divergent selection.

Figure 3-Ecological conditions that can lead to populations of individual specialists versus individual generalists. 


\section{FIGURES}

Figure 1

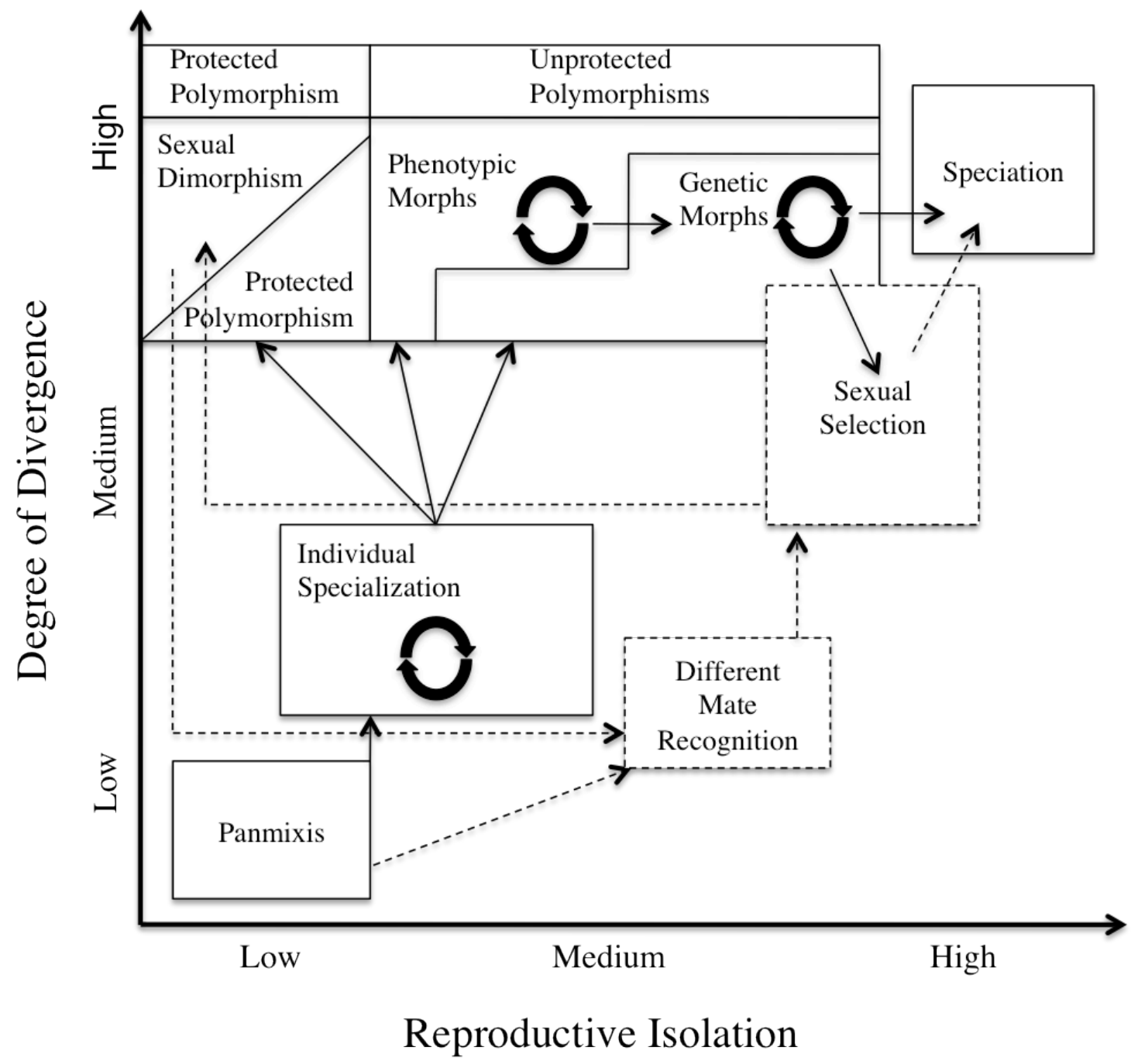


Figure 2
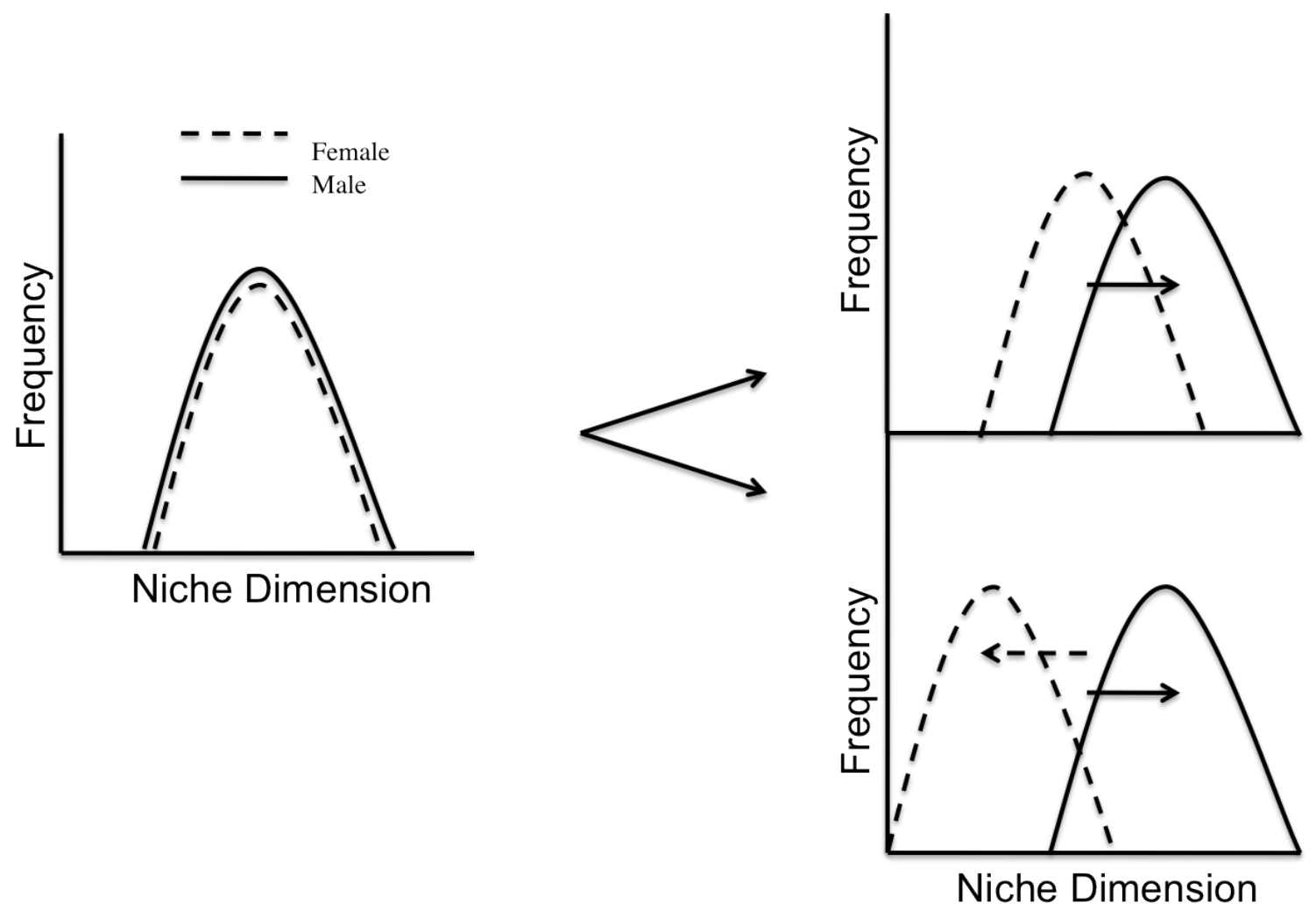
Figure 3

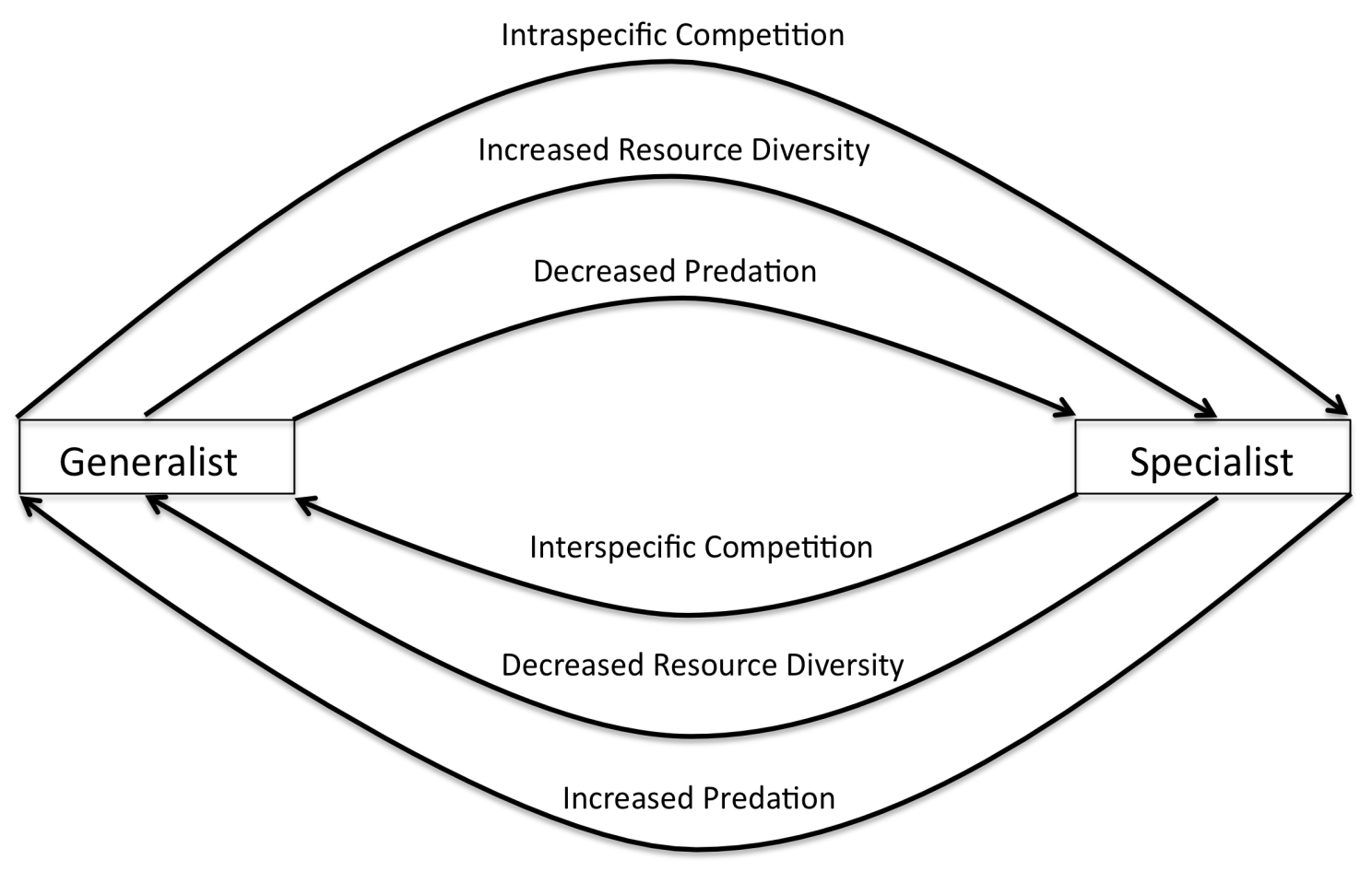




\section{CHAPTER III}

TROPHIC DISCRIMINATION FACTORS AND INCORPORATION RATES

OF CARBON AND NITROGEN STABLE ISOTOPES IN ADULT GREEN FROGS, LITHOBATES CLAMITANS

\section{SUMMARY}

Stable isotope analysis is an increasingly useful ecological tool, but its accuracy depends on quantifying the tissue-specific trophic discrimination factors (TDFs) and isotopic incorporation rates for focal taxa. Despite the technique's ubiquity, most laboratory experiments determining TDFs and incorporation rates have focused on birds, mammals, and fish; we know little about terrestrial ectotherms, and amphibians in particular are understudied. In this study we used two controlled feeding experiments to determine carbon $\left(\mathrm{d}^{13} \mathrm{C}\right)$ and nitrogen $\left(\mathrm{d}^{15} \mathrm{~N}\right)$ isotope TDFs and incorporation rates for skin, whole blood, and bone collagen in adult green frogs, Lithobates clamitans. The mean $( \pm \mathrm{SD}) \mathrm{TDFs}$ for $\mathrm{d}^{13} \mathrm{C}$ were $0.1 \% \circ( \pm 0.4 \%$ ) for skin, $0.5 \% \circ( \pm 0.5 \%$ ) for whole blood, and $1.6 \% \circ\left(0.6 \%\right.$ ) for bone collagen. The mean $( \pm \mathrm{SD})$ TDFs for $\mathrm{d}^{15} \mathrm{~N}$ were $2.3 \%$ $( \pm 0.5 \%$ o for skin, $2.3 \% \circ( \pm 0.4 \%$ ) for whole blood, and $3.1 \%$ ( $\pm 0.6 \%$ ) for bone collagen. A combination of different isotopic incorporation models was best supported by our data. Half-lives for carbon were 89 and 8 days for skin (two-compartment model), 69 days for whole blood, and 332 days for bone collagen. Half-lives for nitrogen were 75 days for 
skin, 71 days for whole blood, and 625 days for bone collagen. Our results help fill a taxonomic gap in our knowledge of stable isotope dynamics and provide ecologists with a method to measure anuran diets at multiple timeframes.

\section{INTRODUCTION}

The use of stable isotopes as natural resource tracers in ecological studies has exploded over the last two decades (Fry 2006; Newsome et al. 2007; Martínez del Rio et al. 2009a; Layman et al. 2012). Although stable isotope analysis is an informative tool, taxon-specific knowledge of two important isotope properties is required to use them accurately in diet analyses. The first property is the degree of isotopic discrimination that occurs between trophic levels (Vanderklift and Ponsard 2003; Fry 2006), creating an offset in the isotope value of the consumer's tissues relative to that of its prey. Such systematic and predictable offsets are often called trophic discrimination factors (TDFs;

Tieszen et al. 1983; Hobson and Clark 1992a; Stegall et al. 2008) and knowing their values is essential to quantifying diet composition via the use of mixing models (Phillips et al. 2005; Moore and Semmens 2008; Parnell et al. 2010). Across vertebrate taxa, these TDFs typically range from -0.4 to $7.9 \%$ for carbon and -0.1 to $4.0 \%$ for nitrogen (Caut et al. 2009), and they often differ among tissues, a phenomenon known as tissue-specific discrimination (Martínez del Rio et al. 2009a).

The second property is the rate of isotopic incorporation and how it differs among metabolically active tissues within a species (DeNiro and Epstein 1978; Tieszen et al. 1983; Martínez del Rio et al. 2009b) and among species with different metabolic 
demands (e.g. ectotherms versus endotherms; Bauchinger and McWilliams 2009; Warne et al. 2010). Some tissues, such as skin and blood plasma, have relatively fast isotopic incorporation rates and thus provide diet information integrated over short time scales (days to weeks) prior to collection (Martínez del Rio et al. 2009a). Other metabolically active tissues, such as bone collagen, incorporate isotopes very slowly (Martínez del Rio et al. 2009a) and thus provide diet information integrated over much longer time scales (years). Incorporation rates, however, can vary among species (Martínez del Rio et al. 2009a), and body size, growth rate, and protein turnover have been shown to affect isotopic incorporation rates (Carleton and Martínez del Rio 2005; MacAvoy et al. 2006; Martínez del Rio et al. 2009a, Murray and Wolf 2013). Thus, isotope-based ecological studies require species- and tissue-specific incorporation rates to estimate the time period to which the ecological information provided by stable isotope analysis pertains. With this information, stable isotope analysis becomes a valuable tool to make ecological inferences and to enable the use of different tissues to quantify dietary and/or habitat switches over time (Tieszen et al. 1983; Hobson and Clark 1992b; Martínez del Rio et al. 2009b).

Trophic discrimination factors and isotopic incorporation rates vary greatly among taxonomic groups (Vander Zanden and Rasmussen 2001; Vanderklift and Ponsard 2003; Caut et al. 2009; Martínez del Rio et al. 2009a). While it is essential to evaluate stable isotope dynamics across diverse species, there is a taxonomic bias in research on TDFs and isotopic incorporation rates within the vertebrates. Most studies have focused on fish, birds, and mammals (fish: Bosley et al. 2002; Logan et al. 2006; Suring and Wing 2009; Carleton and Martinez del Rio 2010; Hussey et al. 2010; Logan and Lutcavage 2010; 
Nelson et al. 2011; Kim et al. 2012; Heady and Moore 2013; birds: Hobson and Clark 1992a,b; Bearhop et al. 2002; Ogden et al. 2004; Cherel et al. 2005; Hobson and Yohannes 2007; Bauchinger and McWilliams 2009; Connan et al. 2014; mammals: Tieszen et al. 1983; Roth and Hobson 2000; Lesage et al. 2002; MacAvoy et al. 2006; Stegall et al. 2008; Florin et al. 2011; Browning et al. 2014). Recent studies have also investigated TDFs and incorporation rates in reptiles (Seminoff et al. 2007; Reich et al. 2008; Fisk et al. 2009; Seminoff et al. 2009; Warne et al. 2010; Murrary and Wolf 2013). However, amphibians have largely been ignored (Dalerum and Angerbjörn 2005; Trakimas et al. 2011). No studies have determined these values for adult anurans and only one study has investigated these values in tadpoles (Caut et al. 2013). However, Caut et al. (2013) used whole tadpoles for their experiment and thus did not determine TDFs or incorporation rates for different tissues.

Here we determine TDFs and isotopic incorporation rates of skin, whole blood, and bone collagen in adult green frogs, Lithobates clamitans, using two controlled feeding experiments in which we fed captive frogs a diet of known isotopic composition. In the first experiment, we fed frogs this diet for a long enough period to be able to determine TDFs for the three tissues. In the second experiment, we tracked isotopic incorporation rates after frogs began eating the laboratory (cricket) diet. With the information provided by these experiments, we not only broaden the general knowledge of stable isotope dynamics in animals, we also provide researchers with a method to determine diet at multiple time frames without lethal harm to anurans. Anurans are a globally threatened taxon (Stuart et al. 2004) that is stressed by habitat loss and degradation (Bonk and Pabijan 2010; Campos et al. 2013), climate change (Barrionuevo and Ponssa 2008; 
McCallum 2010; McCaffery et al. 2012; Murray et al. 2013), and disease (Kilpatrick et al. 2010). A non-lethal method to determine diets and potential diet and/or habitat shifts of frogs and toads brought about by environmental changes will likely prove to be a useful tool for ecologists.

\section{MATERIALS AND METHODS}

Frog Capture and Housing: We used hand nets to capture frogs in September 2011 and July 2013 at Horner Biological Reserve in Oldham County, Kentucky. We immediately placed the frogs inside plastic containers, $20 \mathrm{~cm} \times 14 \mathrm{~cm} \times 12 \mathrm{~cm}$, with shredded coconut husk as substrate and a small bowl of distilled water treated with $0.75 \mathrm{~g} / \mathrm{gallon}$ of aquarium salt. We captured 33 frogs in 2011 and 27 frogs in 2013. At the University of Louisville we kept the frogs in a Precision Scientific Low Temperature Incubator 815 at $23^{\circ} \mathrm{C}$ with an average relative humidity of 55\%. The light:dark cycle in the incubator was 16:8 hours, which mimicked summer in Kentucky. We added more treated distilled water to frogs' water bowls when they were less than half full. The bowls were cleaned and refilled twice a month, and the entire container was cleaned and fresh substrate was provided once a month. All frogs were captured, handled, and housed under the approval of the Institutional Animal Care and Use Committee (UL-IACUC-11015).

Sampling Procedure: Frogs captured on the first trip in 2011 were used in the first controlled feeding experiment to determine the TDFs of whole blood and skin. These frogs were fed one cricket per day for 470 days. Crickets were maintained in the lab on 
the same proprietary diet they were fed at the company from which we purchased them. The mean $( \pm \mathrm{SD}) \delta^{13} \mathrm{C}$ and $\delta^{15} \mathrm{~N}$ values of these crickets were $-21.0 \% \circ( \pm 1.0 \%$ ) and $3.5 \%$ o $( \pm 0.2 \%$ o), respectively $(\mathrm{N}=10)$. We randomly selected ten frogs and used them to determine the TDFs of whole blood collected from the lingual vein and skin collected from a toe clip. When performing these toe clips, we used the second most outer toe on the front limbs, as these toes do not serve a specific purpose, such as digging or amplexus. Additionally, to alleviate stress and reduce pain, frogs were injected intracoelomically with the amphibian safe analgesia flunixin megluminev.

Frogs from the second trip in 2013 were used in the second controlled feeding experiment to determine the isotopic turnover rates of whole blood, skin, and bone collagen, and to estimate the TDFs for bone collagen (see statistical analysis section below). These frogs were fed two crickets a day that had mean $( \pm \mathrm{SD}) \delta^{13} \mathrm{C}$ and $\delta^{15} \mathrm{~N}$ values of $-20.1 \% \circ( \pm 0.5 \% 0)$ and $4.6 \%$ ( $\pm 0.4 \% o)$, respectively $(\mathrm{N}=15)$. The stable isotope values of the crickets were measured at the beginning $(\mathrm{N}=10)$ and near the end $(\mathrm{N}=5)$ of the experiment and these values showed that the isotope values remained constant over the course of the experiment $\left(\delta^{13} \mathrm{C}: \mathrm{t}=-0.223, \mathrm{df}=12.63, p=0.827 ; \delta^{15} \mathrm{~N}: \mathrm{t}=-0.973, \mathrm{df}\right.$ $=12.63, p=0.3489)$. Because our sampling procedure included a toe clip, we could not sample individual frogs more than twice. We accordingly designed an experimental set up that allowed us to sample each frog twice, once in the first four of the eight sampling days and once in the latter four. Frogs were randomly divided into four groups of six, with three frogs remaining to replace any frogs that died during the experiment. On a sampling day, all six frogs in that group were sampled, expect on day 32 when only four frogs were sampled because of high mortality likely associated with acclimating to the 
laboratory and incubator. Frogs from the first group were sampled on day 0 (the day they were captured) and day 32 after the switch to the laboratory diet. Frogs from the second group were sampled on days 4 and 64 after switching to the laboratory diet. Frogs from the third group were sampled on days 8 and 128, and frogs from the fourth group on days 16 and 256. In total, frogs were sampled on days $0,4,8,16,32,64,128$, and 256 after the diet switch.

Stable Isotope Analysis: Whole blood and clipped toes were dried in the oven at $60^{\circ} \mathrm{C}$ for $\sim 48$ hours. We removed the skin manually and then separated ligaments and tendons from the bone. Bone samples from the toe clips were demineralized in $0.5 \mathrm{~N}$ hydrochloric acid in a refrigerator for $\sim 24 \mathrm{hrs}$, after which they were dried in an oven for 48 hours at $60^{\circ} \mathrm{C}$. We lipid-extracted bone collagen via three 24 -hour soaks in a 2:1 chloroform:methanol solution, after which the bone collagen was thoroughly rinsed in distilled water and dried in the oven for $\sim 48$ hours at $60^{\circ} \mathrm{C}$. We weighed sub-samples of whole blood, skin, and bone collagen to $\sim 0.5 \mathrm{mg}$ on a Mettler Toledo AG245 micro-scale and placed them in $5 \times 3.5 \mathrm{~mm}$ tin capsules. Carbon $\left(\delta^{13} \mathrm{C}\right)$ and nitrogen $\left(\delta^{15} \mathrm{~N}\right)$ isotope values were measured at the University of New Mexico Center for Stable Isotopes (Albuquerque, NM). The samples were combusted in a Costech 4010 elemental analyzer (Costech, Valenicia, CA) coupled to a Thermo Scientific Delta V mass spectrometer (Thermo Scientific, Bremen, Germany). Stable isotope values are expressed using delta notation $(\delta)$ in parts per thousand $(\%)$, where $\delta X=\left(\mathrm{R}_{\text {sample }} / \mathrm{R}_{\text {standard }}-1\right)^{*} 1000$, with $\mathrm{R}_{\text {sample }}$ and $\mathrm{R}_{\text {standard }}$ the molar ratios of $\mathrm{C}^{13} / \mathrm{C}^{12}$ and $\mathrm{N}^{15} / \mathrm{N}^{14}$ of the sample and the standard reference material. The reference material was Vienna-Pee Dee Belemnite for carbon and 
atmospheric $\mathrm{N}_{2}$ for nitrogen. Repeated analysis of in-house reference materials of similar composition as the tissue we analyzed showed that precision (SD) for $\delta^{13} \mathrm{C}$ and $\delta^{15} \mathrm{~N}$ values was $\pm 0.2 \%$.

Statistical Analysis: We calculated TDFs as the difference between the $\delta^{13} \mathrm{C}$ or $\delta^{15} \mathrm{~N}$ value of the consumer's tissues and that of its diet, e.g., $\delta^{13} C_{\text {tissue }}-\delta^{13} C_{\text {diet }}$, which is also commonly denoted as $\mathrm{D}^{13} \mathrm{C}_{\text {tissue-diet }}$. We used an ANOVA and a post-hoc Tukey's honest significant differences test to examine differences between the TDFs of each tissue type. The TDF of bone collagen was estimated from the second controlled feeding experiment. To derive this estimate, we first had to determine the isotopic values of the wild frog diet. Because the diet of wild green frogs varies little over time $\left(\delta^{13} \mathrm{C}\right.$ range: $1.3 \%$; $; \delta^{15} \mathrm{~N}$ range: $1.4 \% \circ ; \mathrm{N}=100$ frogs), we assumed that the isotopic values of their diet similarly varied little over time. Any differences in isotopic values across tissue types should thus have resulted from tissue differences in TDFs. Accordingly, to determine the wild frog diet, we subtracted the TDFs of $\mathrm{C}$ and $\mathrm{N}$ for skin and blood from the isotope values of these tissues in Group 1 on Day 0 (i.e. the tissue values based on wild diet); we averaged these two values for $\mathrm{C}$ and $\mathrm{N}$ to obtain the baseline isotopic values for wild frogs' diet. We then determined the TDF of bone collagen by finding the difference between the bone collagen isotope values of frogs from group 1 on day 0 and the calculated resource baselines. We used those TDFs to estimate the final stable isotope value for bone collagen by adding the TDFs of bone collagen to the stable isotope values of the laboratory cricket diet $\left(\delta^{13} \mathrm{C}=-20.1 \%\right.$ and $\delta^{15} \mathrm{~N}=4.6 \%$ ). 
To model isotopic incorporation we followed the procedure of Cerling et al. (2007) and Martínez del Rio and Anderson-Sprecher (2008). We first calculated the reaction progress variable, which can help determine what type of model best fits the incorporation data. We also used the slopes of these reaction progress variables as starting values of $k$ and $f$ (see equations below) in iterative, non-linear fitting routines. We constructed three models per tissue for both $\delta^{13} \mathrm{C}$ and $\delta^{15} \mathrm{~N}$. The first was a onecompartment model:

$$
\delta \mathrm{X}_{t}=\delta \mathrm{X}_{\infty}-\left(\delta \mathrm{X}_{\infty}-\delta \mathrm{X}_{0}\right) e^{-\mathrm{kt}},(1)
$$

where $\delta X_{t}$ is the isotopic composition at time $t, \delta X_{\infty}$ is the isotopic composition at equilibrium on the new diet, $\delta \mathrm{X}_{0}$ is the initial stable isotope composition before the switch to a captive diet, and $k$ is the fractional rate of isotopic incorporation. The second was a two-compartment model:

$$
\delta \mathrm{X}_{t}=\delta \mathrm{X}_{\infty}-\left(\delta \mathrm{X}_{\infty}-\delta \mathrm{X}_{0}\right)\left\{\mathrm{p} e^{-\mathrm{kt}}+\left[(1-\mathrm{p}) e^{-\mathrm{ft}}\right]\right\},(2)
$$

where $p$ is the fractional contribution to the first compartment and $f$ is the fractional rate of isotopic incorporation for the second compartment. The third was a delayedresponse model, in which there is a measurable delay $d$ in days between the diet switch and the incorporation of new isotopes into the tissue:

$$
\delta \mathrm{X}_{t}=\delta \mathrm{X}_{\infty}-\left(\delta \mathrm{X}_{\infty}-\delta \mathrm{X}_{0}\right) e^{-\mathrm{k}(\mathrm{t}-\mathrm{d})}
$$

To determine which of the three models best fit the data, we used Akaike Information Criterion corrected for small sample size ( $\mathrm{AIC}_{c}$; Burnham and Anderson 2002). The model that produced the smallest $\mathrm{AIC}_{\mathrm{c}}$ value was considered the model of best fit. We 
used a linear model to test if frogs grew during our experiment, and found that frogs did not increase in weight between the day they were captured and the second day on which that they were sampled $(\mathrm{F}=0.0004, \mathrm{df}=1,20, p=0.98)$. We therefore did not consider growth in our models and assumed that all change was due to tissue maintenance. We calculated the half-life values of each tissue as $\ln (2) / k$ (Cerling et al. 2007). To test for differences in isotopic incorporation rate between tissues, we ran two linear models in which $\delta^{13} \mathrm{C}$ and $\delta^{15} \mathrm{~N}$ values were the response variables and sample day, tissue type, and a sample day/tissue type interaction were the explanatory variables. All statistical tests and calculations were done in R (R Core Development Team 2013).

\section{RESULTS}

Trophic Discrimination Factors: Controlled Feeding Experiment \#1: For $\delta^{13} \mathrm{C}$, skin had the lowest TDF and bone collagen the highest (table 1). There was a significant overall difference in TDFs among tissues for $\delta^{13} \mathrm{C}(\mathrm{F}=18.58, \mathrm{df}=2,22, p<0.001)$. The TDFs for $\delta^{13} \mathrm{C}$ differed significantly between whole blood and bone collagen $(p<0.001)$ and skin and bone collagen $(p<0.001)$. There was no significant difference in TDFs between whole blood and skin $(p=0.199)$. For $\delta^{15} \mathrm{~N}$, blood and skin had similar TDFs, which were lower than the TDF for bone collagen (table 1). There was a significant overall difference among TDFs for $\delta^{15} \mathrm{~N}(\mathrm{~F}=6.218, \mathrm{df}=2,22, p=0.007)$. The TDFs were significantly higher in bone collagen than in whole blood $(p=0.009)$ or skin $(p=0.012)$ but did not differ significantly between whole blood and skin $(p=0.9919)$. 
Isotopic Incorporation Rates: Controlled Feeding Experiment \#2: For $\delta^{13} \mathrm{C}$, whole blood had the fastest incorporation rate, followed closely by skin and much more distantly by bone collagen (tables 1, 2; fig. 1). For skin and whole blood, $\delta^{13} \mathrm{C}$ values of the samples collected on day 256 were within a standard deviation's length of the mean TDFs from the resource (tables 1,2), indicating that they were approaching their equilibrium values. We therefore felt confident that our asymptote estimate of $-18.7 \%$ for $\delta^{13} \mathrm{C}$ in bone collagen was a fair representation of the equilibrium value. A two-compartment model best described isotopic incorporation in skin (table 3). Delayed-response models best described whole blood and bone collagen data (table 3), where the delay for whole blood was 4 days and the delay for bone collagen was 32 days (table 1). The global linear model that tested for differences in $\delta^{13} \mathrm{C}$ values across tissues and over time was significant $\left(\mathrm{F}=120.2, \mathrm{df}=5,123, p<0.001, \mathrm{R}^{2}=0.823\right)$. Values of $\delta^{13} \mathrm{C}$ changed significantly over the course of the experiment $(\mathrm{t}=17.067, p<0.001)$. Whole blood values of $\delta^{13} \mathrm{C}$ were significantly different from those of bone collagen $(\mathrm{t}=5.121, p<0.001)$ but not from those of skin $(\mathrm{t}=2.409, p=0.843)$. Finally, $\delta^{13} \mathrm{C}$ in whole blood was incorporated at a significantly faster rate than $\delta^{13} \mathrm{C}$ in bone collagen $(\mathrm{t}=-7.163, p<0.001)$ but was not significantly different from skin $(\mathrm{t}=-0.638, p=0.659)$.

Similar to $\delta^{13} \mathrm{C}$ values, $\delta^{15} \mathrm{~N}$ values of whole blood had the fastest incorporation rate, followed closely by whole blood and distantly by bone collagen (tables 1, 2; fig. 2). The final $\delta^{15} \mathrm{~N}$ values for skin and whole blood were within or close to one standard deviation of the mean TDF from the resource, suggesting that our asymptote estimate of $7.6 \%$ for $\delta^{15} \mathrm{~N}$ in bone collagen was near the equilibrium value. One-compartment models best described nitrogen isotope incorporation for skin (table 3) while delayed- 
response models best fit the data for whole blood and bone collagen. However, the difference in $\triangle \mathrm{AIC}_{\mathrm{c}}$ between the delayed-response model and the one-compartment model for $\delta^{15} \mathrm{~N}$ in all tissues was less than 2 (table 3). The global linear model for $\delta^{15} \mathrm{~N}$ was significant $\left(\mathrm{F}=44.13, \mathrm{df}=5,123, p<0.001, \mathrm{R}^{2}=0.628\right)$, and $\delta^{15} \mathrm{~N}$ changed significantly over the course of the experiment $(\mathrm{t}=9.731, p<0.001)$. Both skin and bone collagen $\delta^{15} \mathrm{~N}$ values were significantly different from those in whole blood (skin: $\mathrm{t}=2.409, p=0.0175$; bone collagen: $\mathrm{t}=5.121, p<0.001)$. Nitrogen isotopes were incorporated significantly faster in whole blood than in bone collagen $(\mathrm{t}=-5.094, p<0.001)$ but incorporation rates did not differ significantly between blood and skin $(\mathrm{t}=0.443, p=0.6588)$.

\section{DISCUSSION}

We found that diet could be traced via $\delta^{13} \mathrm{C}$ and $\delta^{15} \mathrm{~N}$ in adult anurans. The $\delta^{13} \mathrm{C}$ and $\delta^{15} \mathrm{~N}$ TDFs for bone collagen were significantly greater than those for skin and whole blood (table 1). The latter two tissues did not differ significantly from each other for either $\delta^{13} \mathrm{C}$ or $\delta^{15} \mathrm{~N}$ TDFs (table 1). Isotopic incorporation rates did not differ statistically between skin and whole blood of frogs for either $\delta^{13} \mathrm{C}$ or $\delta^{15} \mathrm{~N}$, although isotopic incorporation rates were slightly faster in whole blood than in skin (table 1; figs. 1, 2). Bone collagen incorporated both $\delta^{13} \mathrm{C}$ and $\delta^{15} \mathrm{~N}$ stable isotopes at a much slower rate than did skin or whole blood (table 1; figs. 1,2). In our study species, we estimate that whole blood and skin integrate diet information from $~ 2-3$ months before capture, and bone collagen integrates diet information from $\sim 1-2$ years before capture (table 1; figs. 1,2). 
Thus $\delta^{13} \mathrm{C}$ and $\delta^{15} \mathrm{~N}$ can be used to measure diet for at least two discrete timeframes from a single toe clip collected from wild-caught anurans.

Trophic Discrimination Factors: Trophic discrimination factors can vary greatly among taxonomic groups and even closely related species (Vander Zanden and Rasmussen 2001; Vanderklift and Ponsard 2003). In our experiment, TDFs for $\delta^{13} \mathrm{C}$ in all tissues were within the range of those for other freshwater and predator species, but these values vary widely (Vander Zanden and Rasmussen 2001). Compared to other terrestrial ectotherms, our estimated $\delta^{13} \mathrm{C}$ TDF for skin was higher than that of the lizard species Sceloporus undulatus and Crotaphytus collaris (Warne et al. 2010) but lower than that of corn snakes, Elaphe guttata (Fisk et al. 2009). Our $\delta^{13} \mathrm{C}$ TDF for skin is much lower than that of two species of marine turtles, Caretta caretta (Reich et al. 2008) and Dermochelys coriacea (Seminoff et al. 2009). Our TDFs for $\delta^{13} \mathrm{C}$ in whole blood were lower than the TDFs for this tissue in corn snakes (Fisk et al. 2009), but they are similar to those of the marine turtles (Reich et al. 2008; Seminoff et al. 2009).

The primary reason TDFs for $\delta^{13} \mathrm{C}$ differ among tissues is variation in tissue amino acid composition (Vander Zanden and Rasmussen 2001; O’Brien et al. 2002; Howland et al. 2003; O’Brien et al. 2005; McMahon et al. 2010; Newsome et al. 2014). Essential amino acids undergo little, if any, alteration during assimilation and metabolism and therefore exhibit small differences in $\delta^{13} \mathrm{C}$ between consumers and resources $\left(\mathrm{O}^{\prime}\right.$ Brien et al. 2002; Howland et al. 2003; McMahon et al. 2010). Non-essential amino acids, on the other hand, can be synthesized de novo by prokaryotic consumers and isotopic 
fractionation associated with their alteration and synthesis results in large variation in $\mathrm{d}^{13} \mathrm{C}$ values in both glucogenic and ketogenic amino acids (Hare et al. 1991, O'Brien et al. 2002; Howland et al. 2003; McMahon et al. 2010; Newsome et al. 2011, 2014). Thus $\delta^{13} \mathrm{C}$ values of different tissues and their associated TDFs vary depending on the relative contribution of essential and non-essential amino acids in each tissue type. In mammals, proteinaceous tissues are composed of a greater percentage of non-essential (60-72\%) than essential amino acids (28-40\%) (Newsome et al. 2014; Wolf et al. in review). As a result, the isotopic composition of non-essential amino acids likely play a stronger role in determining TDFs, particularly for carbon isotopes.

We do not have a good understanding of the amino acid composition of frog tissues. However, two studies showed that skin peptides of two ranids, Lithobates palustris and Rana dybowskii, were rich in the essential amino acids arginine and leucine (Basir et al. 2000; Jin et al. 2009), and contained several other essential amino acids including threonine and valine (Basir et al. 2000). The presence of many of these essential amino acids in frog skin provides a possible mechanism for this tissue's small TDF. Similarly, in whole blood a greater concentration of the essential amino acids compared to the nonessential could explain why the whole blood TDF in our frogs was small, and future studies that investigate the amino acid composition of frog blood across taxa would be useful. Bone collagen in frogs has a relatively greater amount of non-essential amino acids, such as alanine, proline, serine, and glutamate (Dohi et al. 2004). The greater proportion of non-esssential amino acids in bone is consistent with the larger TDF in bone in our frogs. 
The TDFs for $\delta^{15} \mathrm{~N}$ in L. clamitans were similar to those of other ureotelic species (Vanderklift and Ponsard 2003). Most of the previous studies on terrestrial ectotherms and marine reptiles did not examine nitrogen isotopes, making comparisons difficult. However, TDFs for $\delta^{15} \mathrm{~N}$ in whole blood of our green frogs were nearly identical to those of freshwater turtles (Seminoff et al. 2007). In our study, there was a greater similarity in nitrogen TDFs than in carbon TDFs when comparing across tissues, which is a common trend in many vertebrates (Bearhop et al. 2002; Lesage et al. 2002; Seminoff et al. 2009). The TDFs for bone collagen were higher than those for skin and whole blood for both $\delta^{15} \mathrm{~N}$ and $\delta^{13} \mathrm{C}$, but the difference between bone collagen and the other tissues was much less for nitrogen than for carbon isotopes (table 1). The similarity of the TDFs for $\delta^{15} \mathrm{~N}$ among ureotelic species is consistent with the idea that the mode of excretion of nitrogenous waste drives these TDFs (Vanderklift and Ponsard 2003); however, mode of excretion does not explain differences in TDFs across tissue types, which may instead result from differences among amino acids.

For $\delta^{15} \mathrm{~N}$ analysis, individual amino acids are typically grouped into two categories, trophic and source amino acids (McClelland and Montoya 2002; Schmidt et al. 2004; Popp et al. 2007; Lorrain et al. 2009). Similar to the non-essential and essential amino acids categories often associated with $\delta^{13} \mathrm{C}$ analysis, trophic amino acids often have large discrimination values and source amino acids have little to no discrimination (McClelland and Montoya 2002; Schmidt et al. 2004; Lorrain et al. 2009). Trophic and source amino acids can vary among species (Bloomfield et al. 2011; Hoen et al. 2014), and in order to determine which amino acids are trophic and which are source, compound-specific stable isotope analysis will need to be performed on L. clamitans. 
Such analysis may illuminate why TDFs for $\delta^{15} \mathrm{~N}$ differ little among tissues relative to those for $\delta^{13} \mathrm{C}$.

Isotopic Incorporation Rates: We found that whole blood had the fastest incorporation rates for both $\delta^{13} \mathrm{C}$ and $\delta^{15} \mathrm{~N}$ but they were similar to the rates in skin for both isotope systems (tables 1, 2; figs. 1, 2). Bone collagen incorporated $\delta^{13} \mathrm{C}$ and $\delta^{15} \mathrm{~N}$ isotopes at much slower rates (tables 1, 2; figs. 1,2). Given that frogs are indeterminate growers and that healthy, wild frogs will grow slowly over their lifetime, isotopic incorporation would occur during that growth. However, since frogs did not grow appreciably during our experiment, we hypothesize that all of the observed isotopic incorporation was associated with tissue maintenance. Protein turnover rate, and not basal metabolic rate, has been shown to be the principal factor in controlling isotopic incorporation among species (Bearhop et al. 2002; Martínez del Rio et al. 2009a). Our results corroborate others' findings that adult and slow-growing ectotherms have lower incorporation rates than similarly sized adult endotherms (Hobson and Clark 1992b; Bearhop et al. 2002; Seminoff et al. 2007; Fisk et al. 2009; Murray and Wolf 2013; Browning et al. 2014).

Many studies have shown similar incorporation rates for $\delta^{13} \mathrm{C}$ and $\delta^{15} \mathrm{~N}$, including studies of loggerhead turtles, Caretta caretta (Reich et al. 2009), great skuas, Catharacta skua (Bearhop et al. 2002), and winter flounder, Pseudopleuronectes americanus (Bosley et al. 2002). However, in our study, isotopic incorporation rates of $\delta^{13} \mathrm{C}$ and $\delta^{15} \mathrm{~N}$ were similar in skin and whole blood but not in bone collagen (tables 1,2 ), where $\delta^{15} \mathrm{~N}$ was incorporated at half the rate as $\delta^{13} \mathrm{C}$. Others have also found that $\delta^{13} \mathrm{C}$ and $\delta^{15} \mathrm{~N}$ are incorporated at different rates. In juvenile corn snakes (Elaphe guttata), for example, $\delta^{15} \mathrm{~N}$ was incorporated at one-third the rate of $\delta^{13} \mathrm{C}$ in liver, at one-fifth the rate in blood, 
and one-tenth the rate in muscle (Fisk et al. 2009). Natterjack toad (Bufo calamita) tadpoles incorporated $\delta^{15} \mathrm{~N}$ at half the rate of $\delta^{13} \mathrm{C}$, while western spadefoot (Pelobates cultripes) tadpoles had different incorporation rates of $\delta^{13} \mathrm{C}$ and $\delta^{15} \mathrm{~N}$ depending on diet composition (Caut et al. 2013). On a diet of zooplankton, Pelobates cultripes tadpoles also incorporated $\delta^{15} \mathrm{~N}$ at two-thirds the rate of $\delta^{13} \mathrm{C}$, but when fed macrophytes and algae they incorporated $\delta^{13} \mathrm{C}$ at half the rate and one-fifth the rate of $\delta^{15} \mathrm{~N}$, respectively (Caut et al. 2013). These results suggest that $\delta^{13} \mathrm{C}$ and $\delta^{15} \mathrm{~N}$ may not be incorporated in the same way by all species and that diet may influence the relative incorporation rates of $\delta^{13} \mathrm{C}$ and $\delta^{15} \mathrm{~N}$ in different tissues.

Few studies have used the reaction progress variable and $\mathrm{AIC}_{\mathrm{c}}$ to determine best fitting models to quantify isotopic incorporation rates. While these studies frequently find that one-compartment type models best fit the data, it has become clear that more than one type of model is often needed to best explain isotopic incorporation across several tissues even in a single species (Kurle 2009; Warne et al. 2010; Heady and Moore 2013; Murray and Wolf 2013). No one type of model consistently fit our data best (table 3), although one-compartment and delayed-response models generally performed better than two-compartment models. The incorporation rate of $\delta^{13} \mathrm{C}$ for skin was the only rate that was best fit by a two-compartment model (table 3; fig. 1). Delayed response models best fit $\delta^{13} \mathrm{C}$ trends in whole blood and bone collagen. For $\delta^{15} \mathrm{~N}$, delayed response models best fit the data for whole blood and bone collagen while a one-compartment model best fit skin; however, the $\Delta \mathrm{AIC}_{\mathrm{c}}$ values were less than two between these models, (table 3 ), suggesting that for $\delta^{15} \mathrm{~N}$ the one-compartment and delayed response models may explain the data equally well. Overall, the $\Delta \mathrm{AIC}_{\mathrm{c}}$ values are much smaller for $\delta^{15} \mathrm{~N}$ than for $\delta^{13} \mathrm{C}$, 
a difference that may be driven by a larger variance in the $\delta^{15} \mathrm{~N}$ data (table 2; fig. 2). This larger variance suggests that individual variation may be greater for $\delta^{15} \mathrm{~N}$ than for $\delta^{13} \mathrm{C}$ incorporation rates.

While laboratory studies have recently used multi-compartment models in describing isotopic incorporation, less is known about how to interpret these models in ecological field studies. Interpreting single compartment models is much more intuitive, but how are the two half lives of two compartment models combined so the ecologists can draw meaningful conclusions regarding a relevant time frame? Carleton et al. (2008) suggested weighting the half-lives, such that each half-life is multiplied by $p$ or 1- $p$ from equation 3 before being summed. Applying the delayed response models should be similar to the one-compartment models, where the half-life is applied directly. In the delayed response models, the fractional rate of isotopic incorporation, $k$, is higher than in respective onecompartment models, suggesting the delay increases $k$, and the delay does not need to be added to the half life to obtain a suitable timeframe for the stable isotope data.

Our examination of TDFs and isotopic incorporation rates in adult anurans provides useful information for understanding stable isotope dynamics. We help fill a taxonomic gap in our knowledge of TDFs and isotopic incorporation rates. Our results also highlight the complicated process of isotopic incorporation within a species, where different tissues and isotope types can be best described by different models and $\delta^{13} \mathrm{C}$ and $\delta^{15} \mathrm{~N}$ can be incorporated at similar rates in some tissues but not others. In addition, by determining the TDFs and isotopic incorporation rates for adult anurans, we have provided a method for ecologists to measure and monitor diets over multiple time frames. Globally, anurans face many problems and are recognized as one of the most imperiled taxa on the planet 
(Stuart et al. 2004). Anurans are especially vulnerable to novel diseases (Kilpatrick et al. 2010), habitat degradation/loss, and climate change (Barrionuevo and Ponssa 2008; Bonk and Pabijan 2010; McCallum 2010; McCaffery et al. 2012; Campos et al. 2013; Murray et al. 2013). Using stable isotope analyses on skin and bone collagen will allow researchers to identify any dietary response to changes such as habitat loss if the frogs are sampled at appropriate times. Researchers could also use this technique to characterize any habitat shifts that occur in response to habitat change or loss. Furthermore, our results show that researchers can get all this information from a simple toe clip and do not need to obtain any blood, which can be difficult for many small species. 


\section{TABLES}

Table 1: Isotopic incorporation curve of best fit (lowest $\mathrm{AIC}_{\mathrm{c}}$ ), half-lives, and trophic discrimination factors $(\Delta)$ for skin, whole blood, and bone collagen in $\delta^{13} \mathrm{C}$ and $\delta^{15} \mathrm{~N} . * \Delta$ for bone collagen was estimated by establishing a resource baseline before capture with isotope values of skin and whole blood and finding the difference between those baselines and the day 0 samples for $\delta^{13} \mathrm{C}$ and $\delta^{15} \mathrm{~N}$ values in bone collagen.

$\begin{array}{cccc} & \begin{array}{c}\text { Equation of Best Fit } \\ \boldsymbol{\Delta}^{\mathbf{1 3}} \mathbf{C}\end{array} & \mathrm{t}_{1 / 2}(\text { Days }) & \Delta( \pm \mathrm{SD}) \\ \text { Skin } & -19.9-4.9\left(\left(0.82 e^{-0.007816 t}\right)+\left(0.18 e^{-0.087446 t}\right)\right) & \begin{array}{c}1^{\text {st }}: 88.7 \\ 2^{\text {nd }: 7.6}\end{array} & 0.1(0.4) \\ \text { Whole Blood } & -19.7-4.7 e^{-0.009998(t-4)} & 69.3 & 0.5(0.5) \\ \text { Bone Collagen } & -18.7-4.7 e^{-0.002087(\mathrm{t}-32)} & 332.1 & 1.6(0.6)^{*} \\ & \boldsymbol{\Delta}^{\mathbf{1 5} \mathbf{N}} & & \\ \text { Skin } & 6.9-2.6 e^{-0.009285 t} & 74.65 & 2.3(0.5) \\ \text { Whole Blood } & 6.3-2.2 e^{-0.00979(\mathrm{t}-8)} & 70.8 & 2.3(0.4) \\ \text { Bone Collagen } & 7.6-2.7 e^{-0.001109(\mathrm{t}-32)} & 625.0 & 3.1(0.6)^{*}\end{array}$


Table 2: Mean $\delta^{13} \mathrm{C}$ and $\delta^{15} \mathrm{~N}$ values in skin, whole blood, and bone collagen over the course of the feeding experiment.

\begin{tabular}{ccccccc}
$\begin{array}{c}\text { Sample } \\
\text { Day }\end{array}$ & $\begin{array}{c}\text { Skin } \begin{array}{c}\delta^{13} \mathrm{C} \\
\text { Mean }( \pm \mathrm{SD})\end{array} \\
0\end{array}$ & $\begin{array}{c}\text { Whole Blood } \delta^{13} \mathrm{C} \\
\text { Mean } \\
( \pm \mathrm{SD})\end{array}$ & $\begin{array}{c}\text { Bone Collagen } \delta^{13} \mathrm{C} \\
\text { Mean } \\
( \pm \text { SD })\end{array}$ & $\begin{array}{c}\text { Skin } \delta^{15} \mathrm{~N} \text { Mean } \\
( \pm \mathrm{SD})\end{array}$ & $\begin{array}{c}\text { Whole Blood } \delta^{15} \mathrm{~N} \\
\text { Mean } \\
( \pm \text { SD })\end{array}$ & $\begin{array}{c}\text { Bone Collagen } \\
\delta^{15} \mathrm{~N} \text { Mean } \\
( \pm \text { SD })\end{array}$ \\
\hline 0 & $-24.8(0.5)$ & $-24.5(0.5)$ & $-23.3(0.6)$ & $4.3(0.7)$ & $4.1(0.6)$ & $4.9(0.6)$ \\
4 & $-24.8(0.6)$ & $-24.6(0.4)$ & $-23.6(0.5)$ & $4.2(0.2)$ & $4.2(0.5)$ & $5.0(0.5)$ \\
8 & $-24.0(0.2)$ & $-24.2(0.2)$ & $-23.6(0.5)$ & $4.7(0.6)$ & $4.2(0.7)$ & $4.9(1.0)$ \\
16 & $-23.4(0.3)$ & $-23.9(0.1)$ & $-23.5(0.5)$ & $4.8(0.3)$ & $4.5(0.4)$ & $4.9(0.4)$ \\
32 & $-23.2(0.6)$ & $-23.7(0.5)$ & $-23.1(1.1)$ & $4.7(0.5)$ & $4.1(0.3)$ & $4.9(0.2)$ \\
64 & $-22.4(0.4)$ & $-22.3(0.6)$ & $-23.1(0.3)$ & $5.0(0.3)$ & $4.8(0.8)$ & $4.9(0.4)$ \\
128 & $-21.7(0.5)$ & $-21.0(0.3)$ & $-22.3(0.9)$ & $6.4(0.8)$ & $6.0(0.3)$ & $5.4(0.7)$ \\
264 & $-19.9(0.2)$ & $-19.7(0.4)$ & $-21.6(0.2)$ & $6.9(0.4)$ & $6.3(0.3)$ & $5.5(0.7)$ \\
\hline
\end{tabular}


Table 3: $\mathrm{AIC}_{\mathrm{c}}$ and $\triangle \mathrm{AIC}_{\mathrm{c}}$ values for each model across both isotope types and all tissues types. Rows that are in bold indicate best-fitting models for that tissue and isotope type. The $\Delta \mathrm{AIC}_{\mathrm{c}}$ values marked with an asterisk are close enough to the values of models with lowest $\mathrm{AIC}_{\mathrm{c}}$ that the two models may fit the data equally well.

\begin{tabular}{|c|c|c|c|}
\hline Tissue & Model & $\mathrm{AIC}_{\mathrm{c}}$ & $\Delta \mathrm{AIC}_{\mathrm{c}}$ \\
\hline \multicolumn{4}{|c|}{$\delta^{13} \mathrm{C}$} \\
\hline \multicolumn{4}{|l|}{ Skin } \\
\hline & One-compartment & 72.62 & 6.48 \\
\hline & Two-compartment & 66.14 & $\mathbf{0}$ \\
\hline & Delayed response & 80.54 & 14.4 \\
\hline \multicolumn{4}{|c|}{ Whole Blood } \\
\hline & One-compartment & 55.75 & 6.76 \\
\hline & Two-compartment & 58.37 & 9.38 \\
\hline & Delayed response & 48.99 & $\mathbf{0}$ \\
\hline \multicolumn{4}{|c|}{ Bone Collagen } \\
\hline & One-compartment & 76.93 & 3.65 \\
\hline & Two-compartment & 79.28 & 6 \\
\hline & Delayed response & 73.28 & $\mathbf{0}$ \\
\hline \multicolumn{4}{|c|}{$\delta^{15} \mathbf{N}$} \\
\hline \multicolumn{4}{|l|}{ Skin } \\
\hline & One-compartment & 73.35 & $\mathbf{0}$ \\
\hline & Two-compartment & 75.66 & 2.31 \\
\hline & Delayed response & 73.69 & $0.34 *$ \\
\hline \multicolumn{4}{|c|}{ Whole Blood } \\
\hline & One-compartment & 82.71 & $1.54 *$ \\
\hline & Two-compartment & 85.03 & 3.86 \\
\hline & Delayed response & 81.17 & $\mathbf{0}$ \\
\hline \multicolumn{4}{|c|}{ Bone Collagen } \\
\hline & One-compartment & 68.14 & $0.12 *$ \\
\hline & Two-compartment & 70.37 & 2.35 \\
\hline & Delayed response & 68.02 & $\mathbf{0}$ \\
\hline
\end{tabular}




\section{FIGURE LEGEND}

Figure 1 - Isotopic incorporation curves for $\delta^{13} \mathrm{C}$ in a) skin (two compartment model), b) whole blood (delayed response model), and c) bone collagen (delayed response model). Diagnostic reaction progress variable, $\operatorname{Ln}(1-\mathrm{F})$, for d) skin, e) whole blood, and f) bone collagen. See Table 1 for regression equations and half-lives.

Figure 2-Isotopic incorporation curves for $\delta^{15} \mathrm{~N}$ in a) skin (one compartment), b) whole blood (delayed response model), and c) bone collagen (one compartment). Diagnostic reaction progress variable, $\operatorname{Ln}(1-\mathrm{F})$, for d) skin, e) whole blood, and f) bone collagen. See Table 1 for regression equations and half-lives. 


\section{FIGURES}

Figure 1

a)

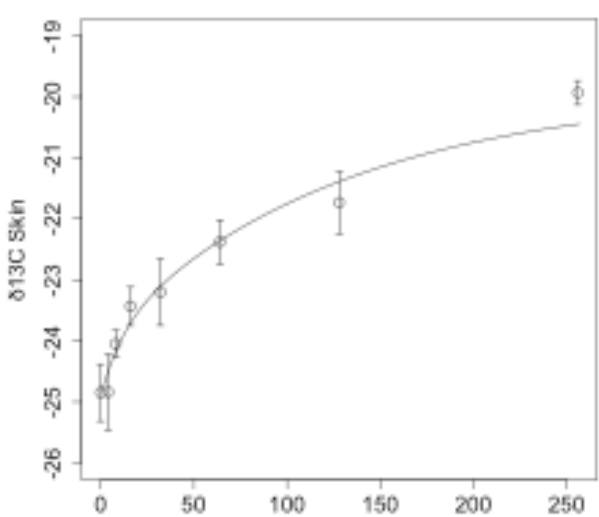

b)

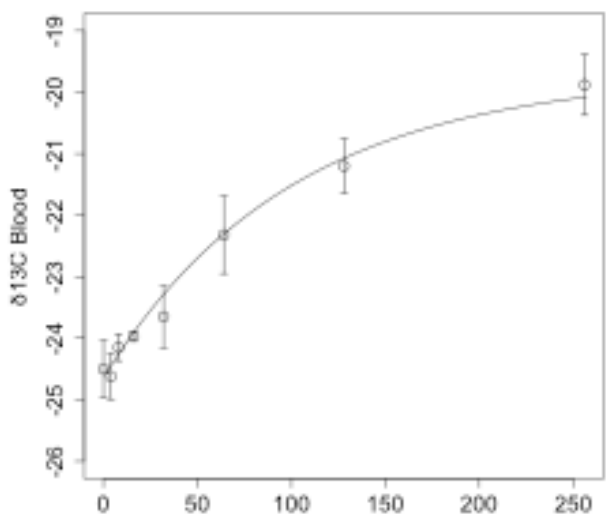

c)

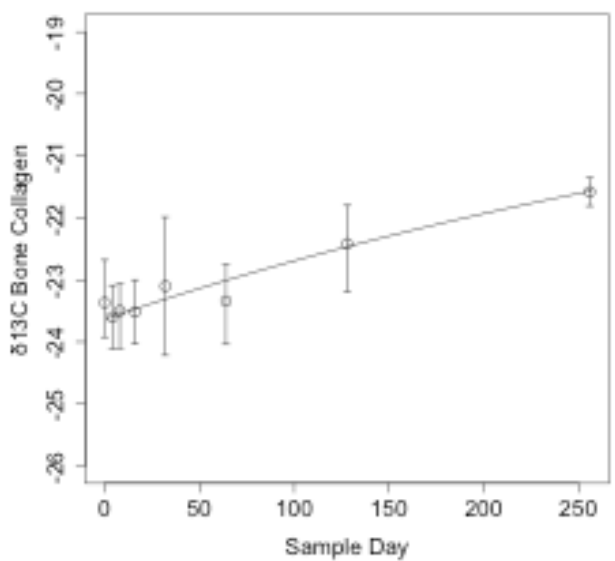

d)

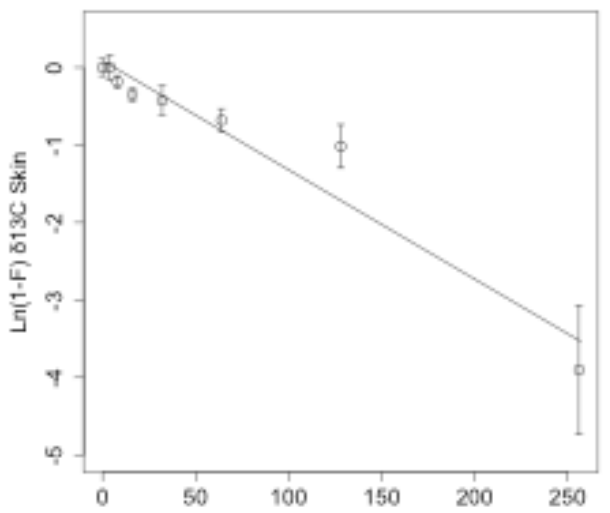

e)

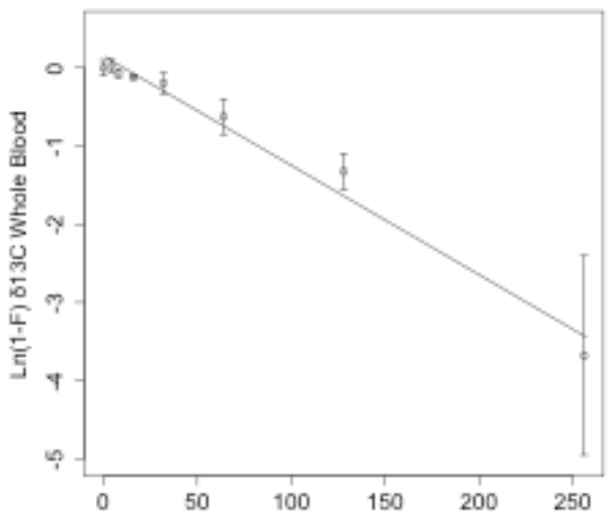

f)

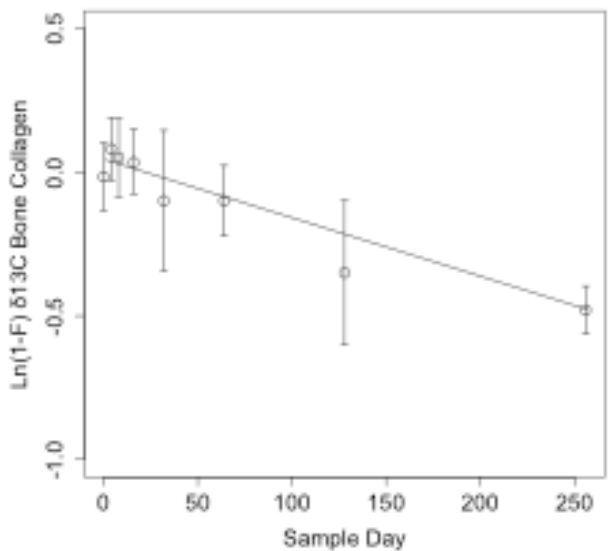


Figure 2

a)

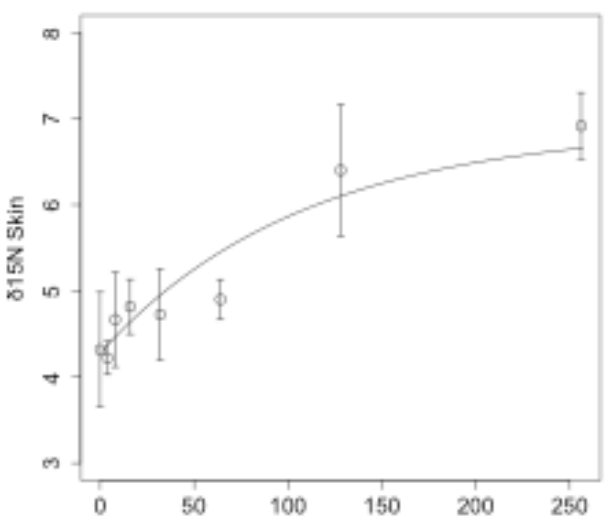

b)

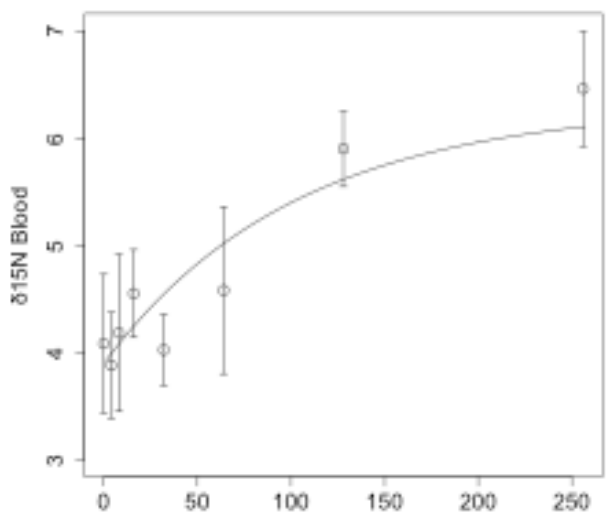

c)

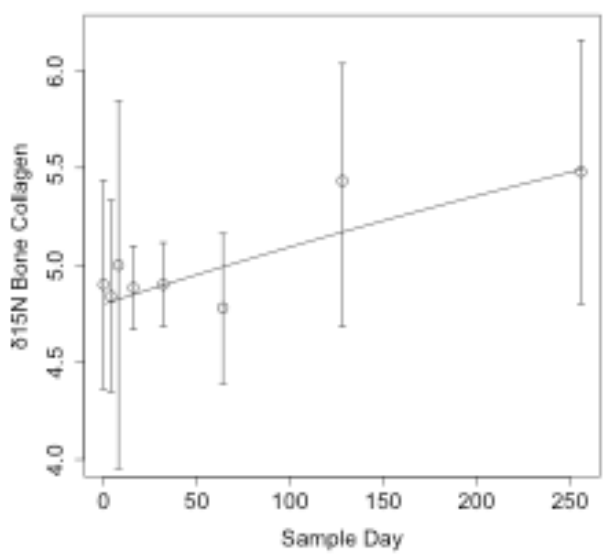

d)

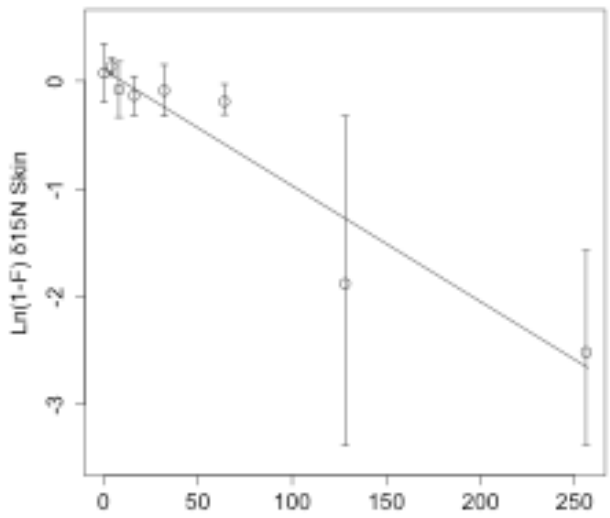

e)

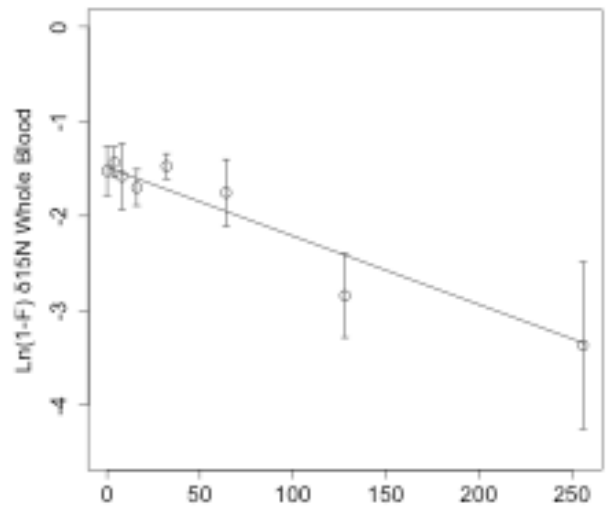

f)

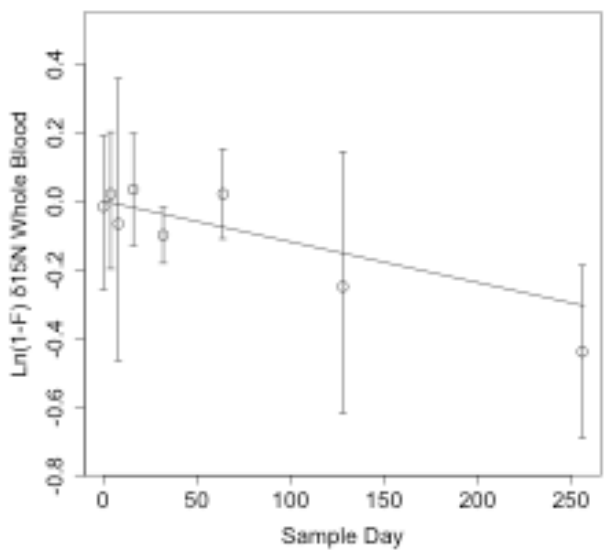




\title{
CHAPTER IV \\ DIFFERENT ECOLOGICAL CONDITIONS SUPPORT INDIVIDUAL SPECIALIZATION IN CLOSELY RELATED AND ECOLOGICALLY SIMILAR SPECIES
}

\begin{abstract}
SUMMARY
Individual differences of diet in generalist foragers has important implications for ecology and evolution. Recent research has shown that individual specialization (IS) is influenced by ecological parameters such as ecological opportunity, intraspecific competition, and interspecific competition. However, the ecological parameters have inconsistent effects on IS. Most studies on IS have focused on a single species, and we are not familiar how ecological parameters affect IS differently in different species. We determined IS in five species of frogs and toads and tested whether IS was influenced by resource diversity, conspecific density, and heterospecific density. We used an AIC approach to determine which parameters best described IS in each species. We found that different ecological factors influenced IS in different species. Resource diversity and conspecific density most frequently were the ecological parameter best at explaining IS. Furthermore, the direction of the relationship was not always in the predicted direction many for all species. These results highlight that the dynamics of IS may differ among
\end{abstract}


even closely related species. Likewise, parameters such as resource diversity and conspecific density do not always have the predicted positive relationship on IS.

\section{INTRODUCTION}

Research over the last decade has demonstrated the importance of individual dietary specialization (IS) in generalist predators (Schluter 2002; Bolnick et al. 2003; Lichstein et al. 2007; Tinker et al. 2008; Schindler et al. 2010; Bolnick et al. 2011; Wennersten and Forsman 2012). At the ecological level, IS helps maintain high biodiversity by increasing population stability (Chamberlain et al. 2005, Schindler et al. 2010) and the likelihood of species coexistence (Lichstein et al. 2007; Bolnick et al. 2011). In addition, IS can affect the movement of energy, breaking links between habitats when individuals use only one habitat type (Quevedo et al.2009) or creating links between habitats when individuals share a common habitat type but differ in their alternative types (Rosenblatt and Heithaus 2011). At the evolutionary level, IS can be the variation that natural selection acts upon to develop resource polymorphisms, which can potentially speciate (Schluter 2002, 2001; Nosil 2012).

Just as IS has ecological consequences, it is also influenced by ecological factors. The best understood of these is intraspecific competition, whose effects on IS have been well studied in both the field and the laboratory (Svanbäck and Bolnick 2005, 2007; Darimont et al. 2009; Bolnick et al. 2010; Frédérich et al. 2010; Martin and Pfennig 2010; Agashe and Bolnick 2010; Tinker et al. 2012, Evangelista et al. 2014). An increase in intraspecific competition can increase IS by driving individuals to use novel or less 
preferred prey types. There are two ways in which a population's niche can expand during times of high intraspecific competition while the widths of individual niches remain similar (Svanbäck and Bolnick 2005). Individuals can differ in their rank order of less preferred prey items or can have the same rank order of prey but differ in when they switch to those alternative prey types depending on the frequency of the top ranked prey (Svanbäck and Bolnick 2005). Network analysis has demonstrated that both types of expansion are possible in natural populations (Araújo et al. 2008; Pires et al. 2011; Tinker et al. 2012). However, intraspecific competition may not always be a diversifying force. When the consumer has particularly strong effects on prey resources, than many prey resources will be depleted before intraspecific competition is high (Jones and Post 2013). Finally, other ecological or genetic complications may prevent intraspecific competition from increasing IS (Parent et al. 2014).

Although theory suggests that interspecific competition will reduce niche widths and IS, emperical evidence is inconclusive (Van Valen 1965; Bolnick et al. 2003; Bolnick et al. 2010; Araújo et al. 2011; Abbey-Lee et al. 2013). Some studies have found a general pattern that populations in depauperate assemblages have more IS (Costa et al. 2008; Darimont et al. 2009). Direct work on individual and population level responses to interspecific competitors is mixed (Bolnick et al. 2010; Abbey-Lee et al. 2013). For example, Bolnick et al. (2010) found that threespined stickleback increased IS when released from competition with cut-throat trout but not when released from competition with prickly sculpin, while Abbey-Lee et al. (2013) found that eastern mosquitofish increased IS when interspecific competition was greater. The possibility of differential effects on species coexistence may explain the discrepancy between theory and patterns 
in natural populations (Lichstein et al. 2007; Bolnick et al. 2011; Lasky et al. 2014). In the former example, the competitive effects of each heterospecific competitor, cut-throat trout and prickly sculpin, may have different effects on the foraging behavior of threespined sticklebacks. Likewise, the high IS in the eastern mosquitofish despite high interspecific competition may be the result of increased species coexistence through IS in this system (Lichstein et al. 2007; Bolnick et al. 2011; Lasky et al. 2014).

Ecological opportunity increases IS (Nosil and Reimchen 2005; Parent and Crespi 2009; Darimont et al. 2009). Ecological opportunity is the availability of resources caused by lack of interspecific competition, low predation, high resource diversity, and other habitat variables. Ecological opportunity is a difficult concept to measure and studies have used allochthonous energy inputs (Darimont et al. 2009; Evangelista et al. 2014), habitat size (Nosil and Reimchen 2005), or have composed an index (Parent and Crespi 2009; Martin and Pfennig 2010). The latter studies included resource diversity as species richness. However, including only resource richness may overlook an important factor of how resource diversity, and thus ecological opportunity, affects IS. Many predators decide which prey items to take based on their frequency in the environment (Pyke et al. 1977; Svanbäck and Bolnick 2005) and a more even distribution of resources may better support IS. Despite this possibly important interaction between ecological opportunity and IS, no one has directly tested how resource diversity influences IS.

Most studies on IS have focused on a single target species. While many of these studies have illuminated the importance of individuality, studies investigating the causes of IS have been conflicting (Svanbäck and Bolnick 2005, 2007; Darimont et al. 2009; Frédérich et al. 2010; Jones and Post 2013; Evangelista et al. 2014; Parent et al. 2014). 
Increased intraspecific competition and ecological opportunity generally increase IS, while increased interspecific competition is thought to decrease IS (Svanbäck and Bolnick 2005, 2007; Darimont et al. 2009; Frédérich et al. 2010). However, as mentioned above, there seem to be fairly frequent exceptions to these patterns (Jones and Post 2013; Evangelista et al. 2014; Parent et al. 2014). A possible explanation is that individuals of different species respond differently to these ecological conditions. Increasing the number of species that are included into these types of studies may help elucidate why individuals of different species react differently to different ecological conditions.

Individual specialization in diet of generalist predators is frequently measured with stable isotopes or with stomach contents. The former method, stable isotope analysis (SIA), is a more robust method than stomach content analysis (SCA) because isotopes are incorporated into tissues over time, offering a longitudinal diet perspective (Bearhop et al. 2004; Matthews and Mazumder 2004; Araújo et al. 2007), while SCA only provides a brief "snapshot" of what the individual consumed just prior to capture. Furthermore, many tissues have different incorporation rates and can be compared to determine how consistent individual diets remain over time (Martínez del Rio et al. 2009). Despite these differences in the ability to accurately measure IS in diet, researchers still use SCA as a method to determine individual diets (Chaouch et al. 2012; Sharpe and Chapman 2014; Salividio et al. 2015; Sánchez-Hernández and Amundsen 2015).

In this study we investigated IS in five species of frogs and toads, Anaxyrus americanus, A.fowleri, Lithobates catesbeianus, L. clamitans, L. sphenocephalus. We tested whether resource diversity and intraspecific and interspecific competition affected IS, which we measured by calculating the dissimilarity of diet within species. We used an 
information criterion framework to compare how well different models explained patterns of IS for each species. Additionally, we compared IS measured with SIA and SCA to determine if the latter overestimates among-individual differences.

\section{MATERIALS AND METHODS}

Study Site: This study was performed at ten small ponds in Bernheim Arboretum and Research Forest, Clermont, Kentucky, U.S.A. Bernheim Arboretum and Research Forest is located in the Knobs region of Kentucky. Ponds varied from $41 \mathrm{~m}^{2}$ to $1329 \mathrm{~m}^{2}$ in size and were situated on ridges. Eight of the ten ponds were in forest interior and two were on the edge between a forest and small grassland. White oak-black oak (Quercus alba-Q. velutina $)$ and white oak-chestnut oak (Q.alba-Q. prinus) were the most common forest types in Bernheim. The most abundant understory plants were Smilax spp., Toxicodendron radicans, Leersia oryzoides, and Microstegium vimineum.

Frog Capture and Processing: We collected frogs and toads from April through September in 2011 and 2012 and from April-June in 2013 and 2014. We captured frogs and toads with hand nets in and around ponds starting 30 minutes after sunset and continuing for an average of 55 minutes. Captured frogs and toads were placed in plastic containers.

We obtained stomach contents with a gentle stomach flushing technique (Solé et al. 2005). We caught any expelled stomach contents in a cup and then stored them in $95 \%$ 
ethanol. The prey present were identified later in the laboratory and used to select appropriate prey items on which to run stable isotope analysis (SIA; Bearhop et al. 2004; Polito et al. 2011). Prey groups for SCA included orthopterans, formicids, coleopterans, miscellaneous flying prey, and miscellaneous non-flying prey. Miscellaneous non-flying prey included spiders and insects from the families Pentatomidae, Reduviidae, Membracidae, as well as other non-flying hemipterans and larval lepidopterans. The miscellaneous flying prey group included flying hymenopterans, most insects in Diptera, flying hemipterans such as Cicadellidae, Cercopidae, adult lepidopterans, and adult odonates. We divided stomach contents into these prey groups because of their frequency in certain species' stomachs.

Frogs and toads were individually marked with a unique combination of toe clips to prevent resampling. Skin and bone from these toe clips was used for SIA. The clipped toes were placed in a chilled cooler in the field, dried for 48 hours at $60^{\circ} \mathrm{C}$, and then stored in a cool, dark drawer in the laboratory. After the toe clip, frogs were released at the site where they were captured. All frogs were captured and handled under the approval of the Institutional Animal Care and Use Committee (UL-IACUC-10037 and UL-IACUC-13026).

Stable Isotope Analysis: We removed the skin manually and then separated ligaments and tendons from the bone. Bone samples from the toe clips were demineralized in $0.5 \mathrm{~N}$ hydrochloric acid in a refrigerator for $\sim 24 \mathrm{hrs,} \mathrm{after} \mathrm{which} \mathrm{they} \mathrm{were} \mathrm{dried} \mathrm{in} \mathrm{an} \mathrm{oven} \mathrm{for}$ 48 hours at $60^{\circ} \mathrm{C}$. We lipid-extracted bone collagen via three 24 -hour soaks in a 2:1 
chloroform:methanol solution, after which the bone collagen was thoroughly rinsed in distilled water and dried in the oven for $\sim 48$ hours at $60^{\circ} \mathrm{C}$. We weighed sub-samples of whole blood, skin, and bone collagen to $\sim 0.5 \mathrm{mg}$ on a Mettler Toledo AG245 micro-scale and placed them in $5 \times 3.5 \mathrm{~mm}$ tin capsules. Carbon $\left(\delta^{13} \mathrm{C}\right)$ and nitrogen $\left(\delta^{15} \mathrm{~N}\right)$ isotope values were measured at the University of New Mexico Center for Stable Isotopes (Albuquerque, NM). The samples were combusted in a Costech 4010 elemental analyzer (Costech, Valenicia, CA) coupled to a Thermo Scientific Delta V mass spectrometer (Thermo Scientific, Bremen, Germany). Stable isotope values are expressed using delta notation $(\delta)$ in parts per thousand (\%o), where $\delta X=\left(\mathrm{R}_{\text {sample }} / \mathrm{R}_{\text {standard }}-1\right) * 1000$, with $\mathrm{R}_{\text {sample }}$ and $\mathrm{R}_{\text {standard }}$ the molar ratios of $\mathrm{C}^{13} / \mathrm{C}^{12}$ and $\mathrm{N}^{15} / \mathrm{N}^{14}$ of the sample and the standard reference material. The reference material was Vienna-Pee Dee Belemnite for carbon and atmospheric $\mathrm{N}_{2}$ for nitrogen. Repeated analysis of in-house reference materials of similar composition as the tissue we analyzed showed that precision (SD) for $\delta^{13} \mathrm{C}$ and $\delta^{15} \mathrm{~N}$ values was $\pm 0.2 \%$.

We used the stable isotope values of prey found in stomach contents to determine prey groups for SIA, which were primarily differentiated by ecological functional group. For the toad species, the groups of arthropod prey included terrestrial herbivores, nonflying terrestrial predators, flying terrestrial predators, and Formica ants. The terrestrial herbivores included several species in the order Orthoptera, herbivorous Coleoptera, adult and larval Lepidoptera, and species from the hemipteran families Cicadellidae (leafhoppers) and Issidae (planthoppers). Non-flying terrestrial predators included several predatory Coleoptera species, parasitic and predatory wasps, and several predatory species from the hemipteran families Pentatomidae, Reduviidae, and Nabidae. Flying 
terrestrial predators included adult odonates and one Asilidae (robber fly) species. For the true frog species, we did not include the Formica ants because they were rarely found in stomach contents, but did add an aquatic category because the frogs were more likely to have aquatic prey in their stomachs and to be found in and around the ponds. The aquatic prey group included a newt species (Notopthalmus viridescens), Lithobates spp. tadpoles, a species of whirlygig beetle (Gyrinus sp.), several species of water boatmen from the hemipteran family Corixidae, and a crayfish (Cambrus sp.). We used the mixing model stable isotope analysis in R (SIAR) to determine the proportion of each prey type for all individuals (Parnell et al. 2010). We used trophic discrimination factors described by Cloyed et al. (In Review).

Prey Collection: To determine resource diversity, we collected arthropods around a pond within 10 days of sampling frogs or toads from that pond. To collect ground dwelling arthropods, we placed pit-fall traps on a $2 \mathrm{~m}$ wide transect that began at the pond's edge and continued $130 \mathrm{~m}$ into the surrounding habitat. Two $7.5 \mathrm{~cm}$ diameter pitfall traps were placed $0-2 \mathrm{~m}, 13-15 \mathrm{~m}, 28-30 \mathrm{~m}, 113-115 \mathrm{~m}$, and 128-130m from each pond; one trap was placed on the left half of the transect and the other on the right. The two traps at each distance were 1-2m from one other. All traps were left open for 48 hours, after which the contents of traps were collected and taken to the laboratory for identification. Within several minutes after contents from pit-fall traps were collected, we took sweep-net samples to collect flying arthropods and arthropods in foliage close to the ground. Each sample consisted of 20 sweep-steps, and samples were taken at $0 \mathrm{~m}, 15 \mathrm{~m}$, $30 \mathrm{~m}, 115 \mathrm{~m}$, and $130 \mathrm{~m}$ from each pond. We did not sample aquatic insects because they 
were the least common prey group in true frog diets. Collected arthropods were taken to the laboratory for identification. We summed prey from all sweep-net and pitfall traps for each pond, calculated the proportion of prey for each prey category, and used these proportions to calculate resource diversity with the Shannon-Weiner index. When calculating these resource diversities, we used arthropods collected at all locations for both toad species and for L. sphenocephalus, but for L. catesbeianus and L. clamitans we used just the arthropods collected up to $30 \mathrm{~m}$ from the pond because these frogs species were never found further than $30 \mathrm{~m}$ from the pond.

Population Surveys: On nights that we sampled ponds for resource diversity, we also performed 20-mintue scan searches for anurans starting 30 minutes after sunset. In these surveys, we searched pond edges and the habitats surrounding the ponds up to $100 \mathrm{~m}$ from the pond edge and counted and identified to species all frogs and toads. We calculated the conspecific densities at ponds by dividing the total number of individuals from each species by the area of the pond. For L. catesbeianus and L.clamitans we calculated heterospecific density by subtracting conspecific density from the total density of $L$. catesbeianus, L. clamitans, and L. sphenocephalus. For both toad species, A. americanus and A.fowleri, we obtained heterospecifice densities by subtracted conspecific density from the total density of A. americanus, A.fowleri, and L. sphenocephelus. To obtaine heterospecific densities for L. sphenocephalus, we totaled the density of all frogs and toads and subtracted L. sphenocephalus density. We calculated heterospecific densities in this way because both L.catesbeianus and L. clamitans do not overlap spatially with both of the toad species and vice versa. Lithobates sphenocephalus, however, overlaps 
spatially with the other true frogs and with the toads.

Statistical Analyses: To test for consistency of individual diets across time we performed Pearson's correlations between skin and bone collagen. In skin, $\delta^{13} \mathrm{C}$ has a half-life around 96 days and $\delta^{15} \mathrm{~N}$ has a half-life around 75 days (Cloyed et al. in review). In bone collagen, $\delta^{13} \mathrm{C}$ has a half-life of 332 days and $\delta^{15} \mathrm{~N}$ has a half-life of 625 days (Cloyed et al. in review). Approximately $29 \%$ of $\delta^{13} \mathrm{C}$ in the two tissues represents the same time period and thus the same diet, and about $12 \%$ of the isotopes of nitrogen represents the same time period. Accordingly, between-tissue correlation coefficients that are approximately 0.29 for $\delta^{13} \mathrm{C}$ and 0.12 for $\delta^{15} \mathrm{~N}$ indicate that the organism has shifted its diet and the isotopic similarity between tissues is due only over lap in time periods. Correlation coefficients significantly higher than these values indicate that individuals have remained similar in diet across time frames.

To measure IS, we grouped each frog and toad species by pond and calculated the proportional dissimilarity (PD) in diet. Proportional dissimilarity was calculated as 1 minus the proportional similarity:

$$
\text { Proportional similarity }=1-0.5 * \Sigma\left(\mathrm{p}_{\mathrm{ij}}-\mathrm{q}_{\mathrm{j}}\right) \text {, }
$$

where $\mathrm{p}_{\mathrm{ij}}$ is the proportion of diet type $j$ in individual $i$ 's diet, and $\mathrm{q}_{\mathrm{j}}$ is the mean proportion of diet type $j$ for the population (Bolnick et al. 2002). The PD values vary from 0 to 1 , where 0 indicates complete overlap between an individual and the population, and 1 indicates no overlap between an individual and the population. We used this measure on data from both SIA and SCA. For the analyses on ecological causes 
of IS, we used isotope data only from skin because we had a greater number of samples of skin than of bone collagen.

To test which ecological parameters affected IS, we constructed general linear models in which populations' average PDs were the response variables and resource diversity, conspecific density, and heterospecific density were the explanatory variables. All explanatory variables were averaged by pond. Seven models were constructed for each species and included all possible combinations of the explanatory variables (Table 1). Due to residuals of the models not meeting the normal assumptions, we estimated the slopes of the relationships and the standard error of those slopes using a MCMC bootstrapping approach, where resampling was done 50,000 times (Manly 2006). We then calculated the $95 \%$ confidence intervals from these bootstrapped estimates. We compared these models within each species using Akaike's Information Criterion corrected for small sample sizes $\left(\mathrm{AIC}_{\mathrm{c}}\right.$; Burnham and Anderson 2002; Johnson and Omland 2004; Stephens et al. 2005). As is standard practice, we considered the model with lowest $\mathrm{AIC}_{\mathrm{c}}$ value to be the model of best fit. We ranked the models based on how well they fit the data by calculating $\Delta \mathrm{AIC}_{\mathrm{c}}: \Delta \mathrm{AIC}_{\mathrm{c}}=\mathrm{AIC}_{\mathrm{i}}-\mathrm{AIC}_{\mathrm{m}}$, where $\mathrm{AIC}_{\mathrm{i}}$ is the $\mathrm{AIC}_{\mathrm{c}}$ value of model $i$ and $\mathrm{AIC}_{\mathrm{m}}$ is the $\mathrm{AIC}_{\mathrm{c}}$ value of the best fitting model. To determine the relative significance of the models, we calculated their normalized Akaike weights $w_{i m}\left(\right.$ Johnson and Omland 2004), where $\mathrm{w}_{\mathrm{im}}=\exp \left(-0.5 * \Delta \mathrm{AIC}_{\mathrm{i}}\right) / \Sigma_{\mathrm{r}=1}^{\mathrm{R}} \exp (-0.5 *$ $\left.\Delta \mathrm{AIC}_{\mathrm{i}}\right)$. Since the normalized weight of the best model for most species was below 0.9 , indicating other models also had substantial support, we performed model averaging (Burnham and Anderson 2002), which provides more robust model variances and increases parameter estimates' reliability. To determine the relative importance of factors 
included in the models, we calculated the normalized Akaike weight for each parameter, $\mathrm{w}_{\mathrm{ip}}$, which is the sum of the $\mathrm{w}_{\mathrm{im}}$ values in which that parameter is present (Gotanda et al., 2009). $\mathrm{A} \mathrm{w}_{\mathrm{ip}}=1$ would indicate a parameter present in all models.

To test for differences in PD values calculated from SIA and SCA, we used a paired samples t-test. For this analysis we used only individuals for which we had information from both SIA and SCA. We calculated the differences in PD values measured from both methods and tested whether those differences overlapped with zero.

\section{RESULTS}

Correlations Between Skin and Bone Collagen: Correlations between skin and bone collagen among the five species varied, but most were significant. Anaxyrus americanus had significant correlations between the two tissues but fairly low correlation coefficients (Fig. 1a; carbon: $\mathrm{t}=3.045, \mathrm{df}=21, p=0.006, \mathrm{r}^{2}=0.55$; nitrogen: $\mathrm{t}=2.15, \mathrm{df}=21, p=$ $\left.0.043, \mathrm{r}^{2}=0.42\right)$. Anaxyrus fowleri likewise had significant correlations but much higher correlation coefficients than A. americanus (Fig 1b; carbon: $\mathrm{t}=5.606$, $\mathrm{df}=31, p<0.001$, $r^{2}=0.71$; nitrogen: $\left.\mathrm{t}=6.787, \mathrm{df}=31, p<0.001, \mathrm{r}^{2}=0.77\right)$. Lithobates catesbeianus had the highest correlation coefficients for both $\delta^{13} \mathrm{C}$ and $\delta^{15} \mathrm{~N}$ values (Fig 1c; carbon: $\mathrm{t}=$ 8.671, df $=64, p<0.001, \mathrm{r}^{2}=0.73 ;$ nitrogen: $\left.\mathrm{t}=13.261, \mathrm{df}=64, p<0.001, \mathrm{r}^{2}=0.86\right)$. While, L. clamitans had a significant correlation in $\delta^{13} \mathrm{C}$ values, the correlation coefficient was low (Fig 1d; $\left.\mathrm{t}=4.048, \mathrm{df}=100, p<0.001, \mathrm{r}^{2}=0.38\right)$. The $\delta^{15} \mathrm{~N}$ values in L. clamitans, on the other hand, were highly correlated (Fig $1 \mathrm{~d} ; \mathrm{t}=10.922$, $\mathrm{df}=100, p<$ 
$\left.0.001, \mathrm{r}^{2}=0.74\right)$. Lithobates sphenocephalus did not have significant correlation between the two tissues for either isotope type (Fig 1e; carbon: $\mathrm{t}=2.049, \mathrm{df}=18, p=0.06, \mathrm{r}^{2}=$ 0.43; nitrogen: $\left.\mathrm{t}=1.666, \mathrm{df}=18, p=0.113, \mathrm{r}^{2}=0.37\right)$.

Degree of IS: The amount of IS varied among species, but overall was low (Table 1). Both of the toads had similar PD values. Anaxyrus americanus had a mean $( \pm \mathrm{SD} ; \mathrm{N}) \mathrm{PD}$ value of $0.08( \pm 0.02 ; \mathrm{N}=54)$, and A. fowleri had a mean of $0.08( \pm 0.01 ; \mathrm{N}=37)$. Among the true frogs, L. catesbeianus had the highest PD values, with a mean of $0.10( \pm$ $0.04 ; \mathrm{N}=68)$, followed by L. sphenocephalus with a mean of $0.08( \pm 0.03 ; \mathrm{N}=38)$, and L. clamitans with a mean of $0.07( \pm 0.01 ; \mathrm{N}=75)$. The amount of variation of isotopes among species also varied (Fig. 2a, b and Fig. 3a-c). In general, both the toad species overlapped in isotopic space and fed mostly on Formica ants, spiders, and terrestrial herbivores (Fig. 2a, b). Lithobates catesbeianus had the most within-species variation in isotopic values and fed mostly on non-flying terrestrial predators, spiders, and flying terrestrial predators (Fig. 3a). The isotopic values of both L. clamitans and $L$. sphenocephalus were all centrally located in the terrestrial prey space, indicating that members of these two species fed fairly evenly amongst those prey groups (Fig. 3b, c).

Ecological Correlates of IS: The parameters included in the best-fit models of PD varied across species. In general, models with only one explanatory variable best fit the data (Table 2). Resource diversity was the only variable in the best-fitting models for $A$. americanus and L. catesbeianus (Table 2a, c). In the case of L. catesbeianus, the best-fit 
model weight was high and the other models were poor predictors of proportional dissimilarity (Table 2c). For A. americanus, the two models that contained only heterospecific or conspecific density were also reasonable predictors of the data (Table 2a). In A.fowleri, the two one-variable models that included conspecific density and resource diversity best explained IS (Table 2b). Heterospecific density best explained patterns of IS in L. clamitans, followed by conspecific density (Table 2d). The model with only conspecific density best explained proportional dissimilarity in $L$. sphenocephalus, but models with conspecific density and resource diversity also had similarly high weights and may explain the data equally well as does conspecific density (Table 2e).

The weights of each variable also differed among species (Table 3). Resource diversity had the highest weights for both A. americanus and L. catesbeianus (Table 3a, c). In L. catesbeianus, the $95 \%$ confidence intervals (CI) of the estimated slope did not include zero (Table 3c), but in A. americanus, the 95\% CI just crossed zero. Conspecific density had the highest weight in A.fowleri, but resource diversity, which had a similar weight, was the only variable for which the $95 \%$ CI of the estimated slope did not cross zero (Table 3b). Conspecific density also had the highest parameter weight for $L$. sphenocephalus, but both resource diversity and conspecific density had fairly high weights as well (Table 3e). Heterospecific density was by far the highest weighted parameter for L. clamitans and the estimated $95 \%$ CI of the slope of this parameter did not cross zero (Table $3 \mathrm{~d})$.

The relationships between IS and the ecological parameters (resource diversity and conspecific and heterospecific density) were not always consistent or in the predicted 
direction. Resource diversity in A. americanus, A. fowleri, and L. catesbeianus had the predicted positive relationship with IS (Fig. 4a, b, c). However, there was a negative relationship between resource diversity and IS in both $L$. clamitans and $L$. sphenocephalus (Fig. 4d, e). Conspecific density in A. americanus and A. fowleri had the predicted positive relationship with IS (Fig. 5a, b), but there was almost no trend in either L. clamitans or L. sphenocephalus (Fig. 5d, e). Interestingly, there was a fairly strong negative relationship between conspecific density and IS in L. catesbeianus (Fig. 5c). Heterospecific density in L. catesbeianus, L. clamitans, and L. sphenocephalus had the predicted negative relationship with IS (Fig 6c, d, e), but there was no relationship between these two factors in A.fowleri (Fig. 6b) and there was a slightly positive one in A. americanus (Fig 6a).

Comparison of SCA and SIA: In all species, the PD values were much greater when calculated from stomach contents than when calculated from the results from stable isotope mixing models (Fig. 7; A. americanus: $\mathrm{t}=12.78$, $\mathrm{df}=29, p<0.001 ;$ A. fowleri: $\mathrm{t}$ $=8.36, \mathrm{df}=21, p<0.001 ;$ L. catesbeianus: $\mathrm{t}=9.45, \mathrm{df}=24, p<0.001 ;$ L. clamitans: $\mathrm{t}=$ 13.6, $\mathrm{df}=34, p<0.001 ;$ L. sphenocephalus: $\mathrm{t}=6.23, \mathrm{df}=10, p<0.001)$.

\section{DISCUSSION}

The amount of IS varied among frog species but overall was relatively low when measured with SIA and high when measured with SCA (Fig. 7). Furthermore, the relative amount of IS varied widely for one species depending on whether the calculation of IS 
was based on SIA or SCA. Lithobates catesbeianus had the most between-individual diet variation calculated from SIA and the second most when calculated from SCA, but its sister species, L. clamitans, had the least between-individual variation when measured with SIA and the most when measured with SCA (Fig. 7). Lithobates sphenocephalus and A. americanus had similar levels of IS and ranked second or third based on either measure. The other toad species, A. americanus had the second most individual variation when measured with SIA and the third most when measured with SCA, while A.fowleri had the second lowest when measured with SIA and the lowest when measured with SCA (Fig. 7). In addition to the amount of IS varying among species, the ecological parameters that most affected IS also varied among species, but most commonly included resource diversity and conspecific density.

Comparing Isotopes from Different Tissues: In our study, we compared short-term isotopic data from skin to the long-term data from bone collagen (Fig. 1). All species except L. sphenocephalus had statistically significant relationships between skin and bone collagen in both $\delta^{13} \mathrm{C}$ and $\delta^{15} \mathrm{~N}$ values. However, the correlation coefficient varied considerably among species. Lithobates catesbeianus had the highest correlation coefficients, indicating that individuals were consistent in diet over time (Fig. 1c). The $\delta^{13} \mathrm{C}$ and $\delta^{15} \mathrm{~N}$ values in L. catesbeianus were greater in skin than in bone tissue, indicating an increase in terrestrial habitat use and trophic level in more recent diets, but individuals that had high isotope values in bone collagen also had high isotope values in skin (Fig. 1c; Cloyed and Eason In Prep). For $\delta^{13} \mathrm{C}$ values, the lower values in bone collagen may represent isotopic memory from the aquatic stage of their life history 
(Perkins and Speakman 2001). This isotopic memory is more detectable in $\delta^{13} \mathrm{C}$ than in $\delta^{15} \mathrm{~N}$ because $\delta^{13} \mathrm{C}$ values differ between terrestrial and aquatic habitats more than $\delta^{15} \mathrm{~N}$. Anaxyrus fowleri also had high correlation coefficients and indicated that individual's diets remained similar between the two tissue types (Fig. 1b). In A.fowleri, many individuals had higher $\delta^{13} \mathrm{C}$ values in skin and the lower values in bone collagen likely represent isotopic memory. Values of $\delta^{15} \mathrm{~N}$, on the other hand, were very similar between the two tissues (Fig. 1b). Lithobates clamitans had lower correlation coefficients for $\delta^{13} \mathrm{C}$ values, indicating that individuals may not stay consistent in their diet choice over time (Fig. 1d). Values of $\delta^{15} \mathrm{~N}$ were highly correlated, even if shifting up in skin (Fig. 1d). This increase in $\delta^{15} \mathrm{~N}$ values demonstrated that individual frogs increase their trophic level throughout their lives (Post 2002). Anaxyrus americanus, while having relatively more IS when measured through SIA and SCA, had relatively lower correlation coefficients. Anaxyrus americanus had higher $\delta^{13} \mathrm{C}$ and $\delta^{15} \mathrm{~N}$ values for skin. Like $A$. fowleri, lower values of $\delta^{13} \mathrm{C}$ in bone collagen were likely isotopes in the tissue from the aquatic stage of their life history and the higher values in skin represent the more terrestrial diet that they consume as adults. For $\delta^{15} \mathrm{~N}$ values, A. americanus had much higher values in skin than carbon, indicating that individuals increased the trophic level at which they feed throughout their adult lives (Post 2002). Lithobates sphenocephalus had low correlation coefficients and followed no particular pattern in isotopes between skin and bone collagen (Fig. 1e) and therefore likely change their diet between the two time frames represented by skin and bone collagen.

Ecological Causes of IS: Previous studies have identified several factors that 
influence IS-resource diversity, intraspecific and interspecific competion -but often yield conflicting results among species. For example, while most studies have found that IS is greater in areas that have more resources or a more heterogeneous habitat (Darimont et al. 2009; Parent and Crespi 2009; Martin and Pfennig 2010; Matich et al. 2011), some species do not respond to ecological opportunity and resource diversity in the same way (Evangelista et al. 2014). When intraspecific competition is high, some studies found that IS increased (Svanbäck and Bolnick 2007; Tinker et al. 2012; Darimont et al. 2009; Frédérich et al. 2010) but others found that IS decreased (Jones and Post 2013; Parent et al. 2014). Studies on the relationship between interspecific competition and IS have also been inconclusive. On the one hand, many studies have found that when fewer heterospecific competitors are present, the diet of a species or population is more variable, resulting in a wider niche (Costa et al. 2008; Darimont et al. 2009). The adaptive radiation of lacustrine fishes of the northern latitudes is believed to be the result of only a few fish species colonizing new lakes at the glacial retreat and developing morphotypes adapted to the different microhabitats (Schulter 2000), but direct tests have found that the relationship between interspecific competition and IS is species specific and not all species respond in to heterospecific competitors the same way (Bolnick et al. 2010; Abbey-Lee et al. 2013). Despite the inconsistency of how these ecological parameters affect IS, our study is the first to compare these parameters amongst a suite of ecologically similar and evolutionarily related species.

We found that the models that best explained the patterns of IS for each species included single ecological factors (Table 2). In general, resource diversity had the greatest effect on IS among species. For A. americanus and L. catesbeianus, the best- 
fitting model included only resource diversity (Table 2a, c). In addition, while the model with the highest weight for A. fowleri included only conspecific density, it had a similar $\mathrm{AIC}_{\mathrm{c}}$ value to the model that only included resource diversity (Table $2 \mathrm{~b}$ ). Indeed, resource diversity in A. fowleri was the only parameter that had an estimated slope where the $95 \%$ CI did not cross zero (Table $3 b$ ). While both conspecific density and resource diversity may be important in determining IS in A. fowleri, resource diversity may have a more significant effect (Figs. 4b, 5b). Heterospecific density by far had the highest model and parameter weight for Lithobates clamitans and the lowest $\mathrm{AIC}_{\mathrm{c}}$ value (Tables $2 \mathrm{~d}, 3 \mathrm{~d}$ ). The IS in L. sphenocephalus was best explained by conspecific density, but both resource diversity and conspecific density had similar model and parameter weights, as well as similar $\mathrm{AIC}_{\mathrm{c}}$ values (Tables $\left.2 \mathrm{e}, 3 \mathrm{e}\right)$.

Resource diversity had strong effects on IS in the predicted positive direction for $A$. americanus, A.fowleri, and L. catesbeianus (Figs. 5a, b, c and 6a, b, c). Both A. americanus and A.fowleri are active foragers that move around in search of prey (Wells 2007), and L. catesbeianus is a sit-and-wait predator (Bury and Whelan 1986). Resource diversity, however, may act in the same way to increase IS in both foraging modes. For toads that actively move around, greater prey diversity will result in more frequently encountered alternative prey. Likewise, for L. catesbeianus that sit and wait for prey to come to them, higher resource diversity will result in more alternative prey passing through more frequently. Different individuals may select prey depending upon the prey's shape and how it moves (Ewart 1974; 2004). As such, the importance of resource diversity on IS may be fairly widespread among different taxonomic groups and foraging techniques. However, the positive effect resource diversity has on IS is not universal. For 
both $L$. clamitans and $L$. sphenocephalus there was a negative trend between resource diversity and IS (Fig. 4d, e). The trend in both of these species is slight and there may be no relationship (Table $3 \mathrm{~d}$, e). In the case of $L$. clamitans, there is very little diet variation between individuals and this small amount of variation may not be affected by ecological parameters. While ecological opportunity and its associated resource diversity increase IS in many species (Nosil and Reimchen 2005; Parent and Crespi 2009; Darimont et al. 2009), our results combined with at least one other study demonstrated that it is not ecologically universal (Evangelista et al. 2014).

Conspecific density also had strong effects on several species. In both the toad species, the relationship trended in the predicted direction, where there was greater IS at sites with more conspecifics (Fig. 5a, b). Like many other studies, at sites with greater conspecific density, individuals are forced to specialize on underutilized prey (Svanbäck and Bolnick 2005; Tinker et al. 2012; Pires et al. 2011). However, all the true frogs had a negative relationship between conspecific density and IS. Jones and Post (2013) found that high intraspecific competition can decrease IS when the consumer has strong interaction effects on its prey populations. In these cases, by the time the consumer population reaches high densities, many of the prey will be gone or infrequent enough that they are not worth searching for and all the individuals use the same remaining resources. In the case of the toads, they are both active foragers and their populations are fairly evenly spread across of the landscape (Forester et al. 2006). As a result, they may not reach high enough densities at any given location to have strong effects on their prey populations. Both L. catesbeianus and L. clamitans, on the other hand, do not venture far from the pond and their populations are localized (Werner et al. 1995). As a result they 
may have a stronger interaction effect on their prey. While no studies have directly tested the interaction strength these frogs and toads have on their prey populations, some work on invasive L. catesbeianus populations have suggested that do have strong effects (Wang et al. 2007; Li et al. 2011). Lithobates catesbeianus, though, is known as a superior competitor to many species (Werner et al. 1995; Hecnar and M'Closkey 1997) and may have strong interaction effects while L. clamtians is a less strong competitor and its interactive strength may not be as strong. Furthermore, outliers drive the negative relationship in both species, especially L. catesbeianus (Fig. 5c, d), suggesting that a threshold density of conspecifics may exist. After which that threshold density is past the interaction strength between predator and prey will cause a negative relationship between conspecific density and IS. Finally, L. sphenocephalus is more like toads in that they are active hunters, but they are usually less frequent than the other frogs and therefore may not be greatly affected by conspecific density.

Heterospecific density overall had the weakest effect on IS. Only in L.clamitans did the best-fitting model contain heterospecific density (Table 2d). The strong effect heterospecific density has on PD values for L. clamitans may be driven by the competitive relationship with L. catesbeianus. Lithobates catesbeianus is a superior competitor to L. clamitans (Werner et al. 1995; Hecnar and M'Closky 1997) and represents most of the heterospecifics that L.clamitans will encounter given that both species commonly occur near ponds. At ponds where L. catesbeianus densities were high, L. clamitans may have not had many opportunities to specialize on alternative resources because the larger and competively superior L. catesbeianus was either comsuming them or otherwise preventing L. clamitans from gaining access to these prey. 
Our results help illustrate how interspecific competition affects IS, where there are often species-specific responses (Bolnick et al. 2010; Abbey-Lee et al. 2013). In our system, interspecific competition affects IS only in the species that forages in close proximity to a superior competitor. Overall, heterospecific density had a fairly predictable, negative relationship on PD values for all species expect L. sphenocephalus, which had no negative or positive trend between these parameters (Fig. 6a-e).

Comparing IS with SIA and SCA: Measuring IS when using different methods provided drastically different results (Fig. 7). Neither may give a completely accurate view of between-individual diet variation. In our system, SIA likely underestimated IS because many diverse prey taxa are lumped into broad ecological functional groups. Diet diversity could be reduced when these functional groups are lumped together. On the other hand, SCA likely overestimated IS, because SCA will only capture a snapshot of the individuals' diets and does not provide the longitudinal data that are necessary to assess IS (Bearhop et al. 2004). There is general agreement that SIA is a superior method for calculating IS to SCA and fecal content analysis because it does not incorporate diet data longitudinally (Bolnick et al. 2003; Bearhop et al. 2004; Matthews and Mazumder 2004; Araújo et al. 2007). Additionally, SIA has the advantage of comparing diet data from different times through the use of multiple tissue types (Martínez del Rio et al. 2009).

Studies using SCA have frequently found higher amounts of IS than studies using SIA. For example, diet variation in a larval frog was much greater when measured with 
SCA than with SIA (Schriever and Williams 2013). The PD values for brown trout were high when using SCA (PD = 0.31-0.54; Sánchez-Hernández and Amundsen 2015) but lower when using SIA (PD = 0.14-0.5; Evangelista et al. 2014). This regularity in SCA producing greater PD values is consistent with the idea that SCA and similar methods such as fecal content analysis overestimate IS. However, while SCA may overestimate IS, it is difficult to say how accurate SIA is at measuring it. It is likely that in many systems, such as ours, SIA may underestimates IS. Future studies are needed to determine how accurate SIA is at estimating IS and how consistent that accuracy is among different systems. These studies should combine three or more methods of diet analysis and these could include: fecal content analysis, behavioral analysis, fatty acid analysis, as well as SCA and SIA. Additionally, these studies should include repeated samples of fecal content, behavioral observations, or stomach contents to help determine repeatability of these measures.

Conclusions: Our results have demonstrated that the amount of between individual diet variation can differ among species. In several species, there were high correlations between earlier diets and more recent diets, although this was not always the case. Additionally, different ecological parameters affected between-individual diet variation among species. Resource diversity had a strong effect on three of five species, and heterospecific and conspecific density had weaker effects than did resource diversity. Heterospecific density had a weak to moderate effect on three of five species, and conspecific density had a weak to moderate effect on two of five species. Our results suggest that the ecological parameters that affect IS are species specific, and that it is 
inaccurate to assume that intraspecific competition always increases IS or that interspecific competition always decreases it. Furthermore, ecological opportunity and/or resource diversity may not always result in increased IS. Finally, different methods of measuring diet can result in drastically different measures of IS, and SCA likely overestimates it. However, we do not know how accurate SIA is at measuring IS and more studies are needed to determine its accuracy. 


\section{TABLES}

Table 1: The average proportional dissimilarity $(\mathrm{PD}) \pm$ standard deveiation $(\mathrm{SD})$ at each pond for each species.

\begin{tabular}{|c|c|c|c|c|c|}
\hline & $\mathrm{PD}( \pm \mathrm{SD})$ & $\mathrm{PD}( \pm \mathrm{SD})$ & $\mathrm{PD}( \pm \mathrm{SD})$ & $\mathrm{PD}( \pm \mathrm{SD})$ & $\mathrm{PD}( \pm \mathrm{SD})$ \\
\hline Site & A. americanus & A. fowleri & L. catesbeianus & L. clamitans & L. sphenocephalus \\
\hline Back-of-Nowhere & $0.08(0.001)$ & Not Captured & $0.11(0.0071)$ & $0.08(0.041)$ & $0.07(0.046)$ \\
\hline Cattail Puddle & $0.11(0.048)$ & $0.09(0.063)$ & $0.14(0.065)$ & $0.06(0.04)$ & $0.03(0.017)$ \\
\hline Honeyhole & $0.10(0.044)$ & $0.07(0.05)$ & Not Captured & Not Captured & $0.12(4)$ \\
\hline Mudhole & Not Captured & $0.06(0.21)$ & $0.12(0.051)$ & $0.07(0.024)$ & Not Captured \\
\hline Platform Pond & $0.07(0.041)$ & $0.09(0.049)$ & $0.12(0.041)$ & $0.07(0.044)$ & $0.08(0.045)$ \\
\hline Tannin Pond North & Not Captured & Not Captured & Not Captured & $0.09(0.035)$ & Not Captured \\
\hline Tannin Pond South & Not Captured & Not Captured & Not Captured & $0.05(0.027)$ & $0.08(0.029)$ \\
\hline Vernal Pool & $0.10(0.61)$ & Not Captured & $0.05(0.023)$ & Not Captured & Not Captured \\
\hline Wilson Road South & $0.05(0.024)$ & $0.06(0.031)$ & $0.14(0.023)$ & $0.07(0.03)$ & $0.09(0.045)$ \\
\hline Wilson Road North & $0.06(0.033)$ & $0.07(0.26)$ & $0.05(0.015)$ & $0.07(0.042)$ & $0.09(0.03)$ \\
\hline
\end{tabular}


Table 2: Ecological parameter models for each species. $\mathrm{RD}=$ Resource Diversity, $\mathrm{CD}=$ Conspecific Density, $\mathrm{HD}=$ heterospecific density. The model with the lowest $\mathrm{AIC}_{\mathrm{c}}$ value is model of best fit. $\mathrm{W}_{\mathrm{im}}$ is the model weight and represents the ability of the model to describe the data. Higher model weights indicate a more important model. a) Anaxyrus americanus, b) A.fowleri, c) Lithobates catesbeianus, d) L.clamitans, e) L. sphenocephalus. 
a)

\begin{tabular}{cccc} 
a) & \multicolumn{3}{c}{} \\
\hline Model & $\mathrm{AIC}_{\mathrm{c}}$ & $\Delta \mathrm{AIC}_{\mathrm{c}}$ & $\mathrm{W}_{\mathrm{im}}$ \\
\hline PD RD & -23.88 & 0 & 0.675 \\
PD HD & -21.43 & 2.45 & 0.192 \\
PD CD & -21 & 2.88 & 0.16 \\
PD RD+CD & -9.9 & 13.98 & 0.0006 \\
PD CD+HD & -9.9 & 16.43 & 0.0006 \\
PD RD+HD & -8.28 & 15.60 & 0.0002 \\
PD RD+CD+HD & 31.82 & 55.7 & $8.04 \mathrm{E}-13$
\end{tabular}

b)

\begin{tabular}{cccc} 
b) & \multicolumn{3}{c}{} \\
\hline Model & $\mathrm{AIC}_{\mathrm{c}}$ & $\Delta \mathrm{AIC}_{\mathrm{c}}$ & $\mathrm{W}_{\mathrm{im}}$ \\
\hline $\mathrm{PD} \sim \mathrm{CD}$ & -22.15 & 0 & 0.532 \\
$\mathrm{PD} \sim \mathrm{RD}$ & -21.75 & 0.43 & 0.439 \\
$\mathrm{PD} \sim \mathrm{HD}$ & -16.94 & 5.21 & 0.039 \\
$\mathrm{PD} \sim \mathrm{RD}+\mathrm{CD}$ & 6.9 & 29.05 & $2.636 \mathrm{E}-7$ \\
$\mathrm{PD} \sim \mathrm{CD}+\mathrm{HD}$ & 7.81 & 29.96 & $1.659 \mathrm{E}-7$ \\
$\mathrm{PD} \sim \mathrm{RD}+\mathrm{HD}$ & 8.11 & 29.86 & $1.429 \mathrm{E}-7$ \\
$\mathrm{PD} \sim \mathrm{RD}+\mathrm{CD}+\mathrm{HD}$ & 42 & 64.15 & $6.248-15$
\end{tabular}

c)

\begin{tabular}{cccc} 
c) & \multicolumn{3}{c}{} \\
\hline Model & $\mathrm{AIC}_{\mathrm{c}}$ & $\Delta \mathrm{AIC}_{\mathrm{c}}$ & $\mathrm{W}_{\mathrm{im}}$ \\
\hline $\mathrm{PD} \sim \mathrm{RD}$ & -23.93 & 0 & 0.965 \\
$\mathrm{PD} \sim \mathrm{HD}$ & -16.11 & 7.82 & 0.019 \\
$\mathrm{PD} \sim \mathrm{RD}+\mathrm{HD}$ & -13.92 & 9.38 & 0.006 \\
$\mathrm{PD} \sim \mathrm{CD}$ & -13.71 & 10.22 & 0.006 \\
$\mathrm{PD} \sim \mathrm{RD}+\mathrm{CD}$ & -12.36 & 11.57 & 0.003 \\
$\mathrm{PD} \sim \mathrm{CD}+\mathrm{HD}$ & -6.27 & 17.66 & $3.499 \mathrm{E}-5$ \\
$\mathrm{PD} \sim \mathrm{RD}+\mathrm{CD}+\mathrm{HD}$ & 26.59 & 50.52 & $5.216 \mathrm{E}-12$
\end{tabular}

d)

\begin{tabular}{cccc} 
d) & \multicolumn{3}{c}{} \\
\hline Model & $\mathrm{AIC}_{\mathrm{c}}$ & $\Delta \mathrm{AIC}_{\mathrm{c}}$ & $\mathrm{W}_{\mathrm{im}}$ \\
\hline $\mathrm{PD} \sim \mathrm{HD}$ & -37.86 & 0 & 0.744 \\
$\mathrm{PD} \sim \mathrm{CD}$ & -35.60 & 2.26 & 0.24 \\
$\mathrm{PD} \sim \mathrm{RD}$ & -29.62 & 8.24 & 0.012 \\
$\mathrm{PD} \sim \mathrm{RD}+\mathrm{CD}$ & -26.62 & 11.24 & 0.003 \\
$\mathrm{PD} \sim \mathrm{RD}+\mathrm{HD}$ & -24.21 & 13.84 & 0.001 \\
$\mathrm{PD} \sim \mathrm{CD}+\mathrm{HD}$ & -24.02 & 13.84 & 0.0001 \\
$\mathrm{PD} \sim \mathrm{RD}+\mathrm{CD}+\mathrm{HD}$ & 15.36 & 53.22 & $1.008 \mathrm{E}-11$
\end{tabular}

e)

\begin{tabular}{cccc} 
e) & \multicolumn{3}{c}{} \\
\hline Model & $\mathrm{AIC}_{\mathrm{c}}$ & $\Delta \mathrm{AIC}_{\mathrm{c}}$ & $\mathrm{W}_{\mathrm{im}}$ \\
\hline PD CD & -15.38 & 0 & 0.369 \\
PD RD & -15.25 & 0.13 & 0.345 \\
PD HD & -14.87 & 0.52 & 0.285 \\
PD CD+HD & -2.53 & 12.84 & 0.0006 \\
PD $\sim \mathrm{RD}+\mathrm{CD}$ & -1.32 & 14.06 & 0.0002 \\
PD RD+HD & -1.26 & 14.12 & 0.0003 \\
PD RD+CD+HD & 39.14 & 54.52 & $5.34 \mathrm{E}-13$
\end{tabular}


Table 3: The parameter weights, averaged beta, and 95\% CI of the averaged beta. $\mathrm{W}_{\mathrm{ip}}$ is the Akaike weight for each parameter, which is the sum of the model weight values, $\mathrm{w}_{\mathrm{im}}$, in which that parameter was present. Resource diversity for A.fowleri and L. catesbeianus was the only ecological parameter that did not cross zero. a) Anaxyrus americanus, b) A. fowleri, c) Lithobates catesbeianus, d) L. clamitans, e) L. sphenocephalus. 


\begin{tabular}{|c|c|c|c|}
\hline Parameter & $\mathrm{W}_{\mathrm{ip}}$ & Averaged Beta & $95 \%$ CI \\
\hline Diversity & 0.676 & 0.097 & -0.024 to 0.218 \\
\hline $\begin{array}{l}\text { Conspecific } \\
\text { Density }\end{array}$ & 0.161 & 2.66 & -4.717 to 10.042 \\
\hline $\begin{array}{l}\text { Heterospecific } \\
\text { Density }\end{array}$ & 0.165 & 0.029 & -0.284 to 0.342 \\
\hline \multicolumn{4}{|l|}{ b) } \\
\hline Parameter & $\mathrm{W}_{\text {ip }}$ & Averaged Beta & $95 \% \mathrm{CI}$ \\
\hline Diversity & 0.432 & 0.097 & 0.017 to 0.177 \\
\hline $\begin{array}{l}\text { Conspecific } \\
\text { Density }\end{array}$ & 0.536 & 0.622 & -0.707 to 1.952 \\
\hline $\begin{array}{c}\text { Heterospecific } \\
\text { Density }\end{array}$ & 0.032 & 0.018 & -0.239 to 0.276 \\
\hline \multicolumn{4}{|l|}{ c) } \\
\hline Parameter & $\mathrm{W}_{\mathrm{ip}}$ & Averaged Beta & $95 \%$ CI \\
\hline Diversity & 0.952 & 0.326 & 0.084 to 0.568 \\
\hline $\begin{array}{l}\text { Conspecific } \\
\text { Density }\end{array}$ & 0.009 & -0.412 & -2.555 to 1.729 \\
\hline $\begin{array}{l}\text { Heterospecific } \\
\text { Density }\end{array}$ & 0.051 & -0.133 & -1.097 to 0.832 \\
\hline \multicolumn{4}{|l|}{ d) } \\
\hline Parameter & $W_{\text {ip }}$ & Averaged Beta & $95 \%$ CI \\
\hline Diversity & 0.154 & -0.024 & -0.096 to 0.048 \\
\hline $\begin{array}{l}\text { Conspecific } \\
\text { Density }\end{array}$ & 0.49 & -0.123 & -0.587 to 0.342 \\
\hline $\begin{array}{l}\text { Heterospecific } \\
\text { Density }\end{array}$ & 0.468 & -0.072 & -0.318 to 0.174 \\
\hline \multicolumn{4}{|l|}{ e) } \\
\hline Parameter & $W_{\text {ip }}$ & Averaged Beta & $95 \%$ CI \\
\hline Diversity & 0.162 & -0.048 & -0.223 to 0.128 \\
\hline $\begin{array}{l}\text { Conspecific } \\
\text { Density }\end{array}$ & 0.173 & 0.056 & -1.092 to 1.205 \\
\hline $\begin{array}{l}\text { Heterospecific } \\
\text { Density }\end{array}$ & 0.666 & -0.072 & -0.229 to 0.084 \\
\hline
\end{tabular}




\section{FIGURE LEGENDS}

Figure $1-$ Correlation between carbon (top row) and nitrogen (bottom row) stable isotopes in skin (y-axis) and bone collage (x-axis). The line has a slope of one and intercept of 0 , points above the line mean individuals have shifted their diet recently. a) Anaxyrus americanus, b) A.fowleri, c) Lithobates catesbeianus, d) L.clamitans, e) L. sphenocephalus.

Figure $2-\delta^{13} \mathrm{C}$ and $\delta^{15} \mathrm{~N}$ biplot for a) Anaxyrus americanus and b) A.fowleri.

Figure $3-\delta^{13} \mathrm{C}$ and $\delta^{15} \mathrm{~N}$ biplot for a) Lithobates catesbeianus, b) L.clamitans, and c) L. sphenocephalus.

Figure 4-Resource diversity versus proportional dissimilarity. Each point represents a pond and the line is the slope of the relationship. a) Anaxyrus americanus, b) A.fowleri, c) Lithobates catesbeianus, d) L. clamitans, e) L. sphenocephalus. Proportional dissimilarity calculated from stable isotopes in skin.

Figure 5-Conspecific density versus proportional dissimilarity. Each point represents a pond and the line is the slope of the relationship. a) Anaxyrus americanus, b) A.fowleri, c) Lithobates catesbeianus, d) L. clamitans, e) L. sphenocephalus. Proportional dissimilarity calculated from stable isotopes in skin. 
Figure 6-Heterospecific density versus proportional dissimilarity. Each point represents a pond and the line is the slope of the relationship. a) Anaxyrus americanus, b) A.fowleri, c) Lithobates catesbeianus, d) L. clamitans, e) L. sphenocephalus. Proportional dissimilarity calculated from stable isotopes in skin.

Figure 7-Barplot with proportional dissimilarity values measured with SIA (black bars) and SCA (grey bars). Anam = Anaxyrus americanus, Anfo $=$ A. fowleri, Lica $=$ Lithobates catesbeianus, Licl $=$ L. clamitans, Lisp $=$ L. sphenocephalus . 


\section{FIGURES}

Figure 1
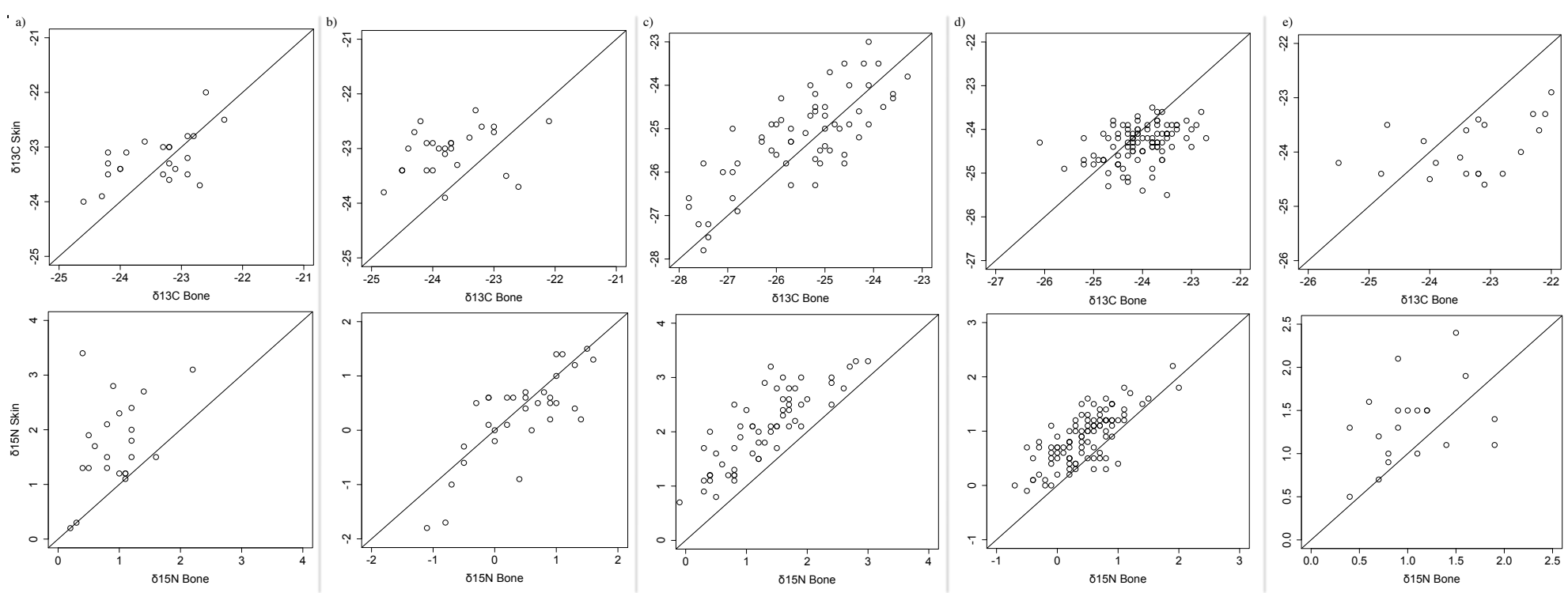
Figure 2
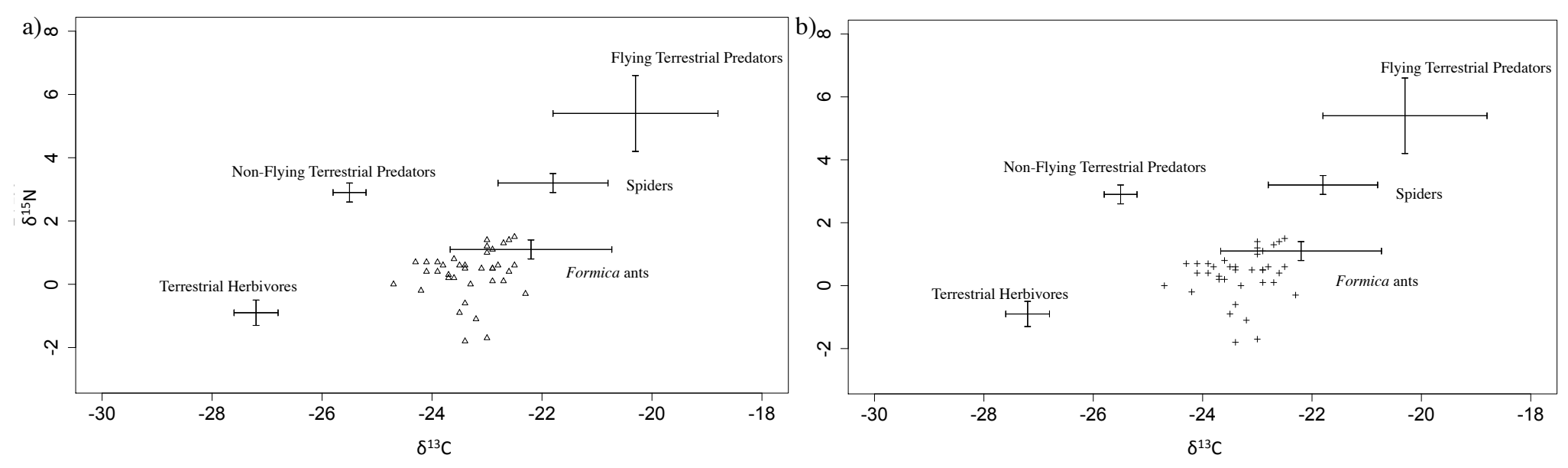
Figure 3
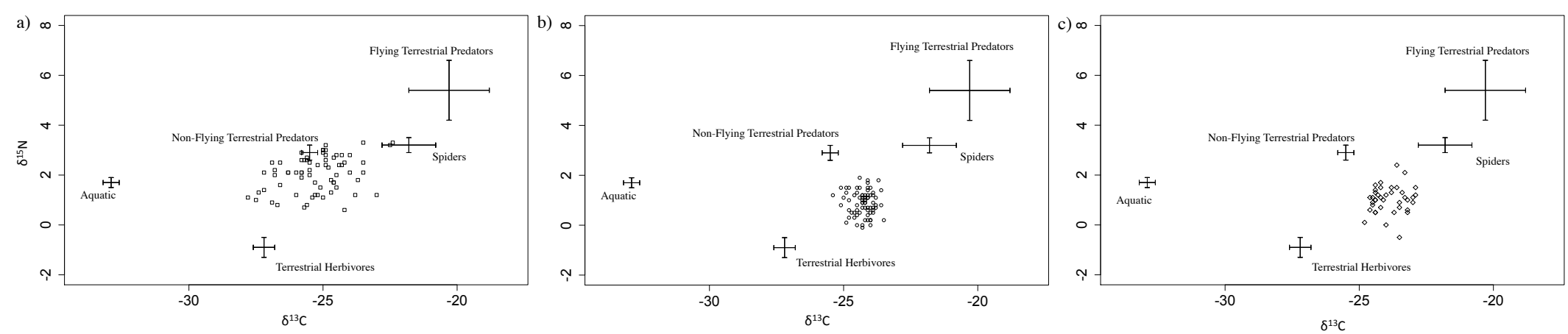
Figure 4
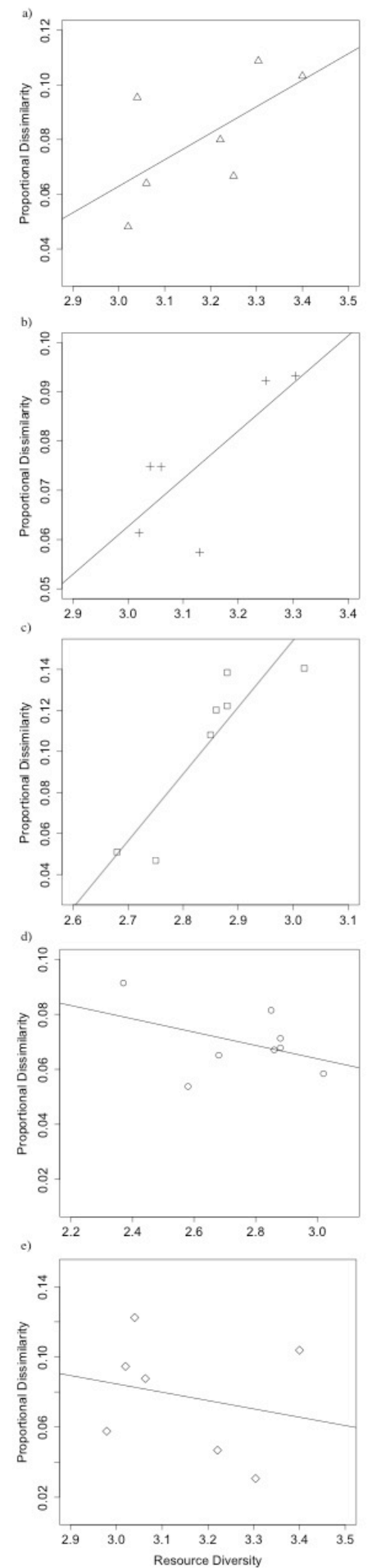
Figure 5
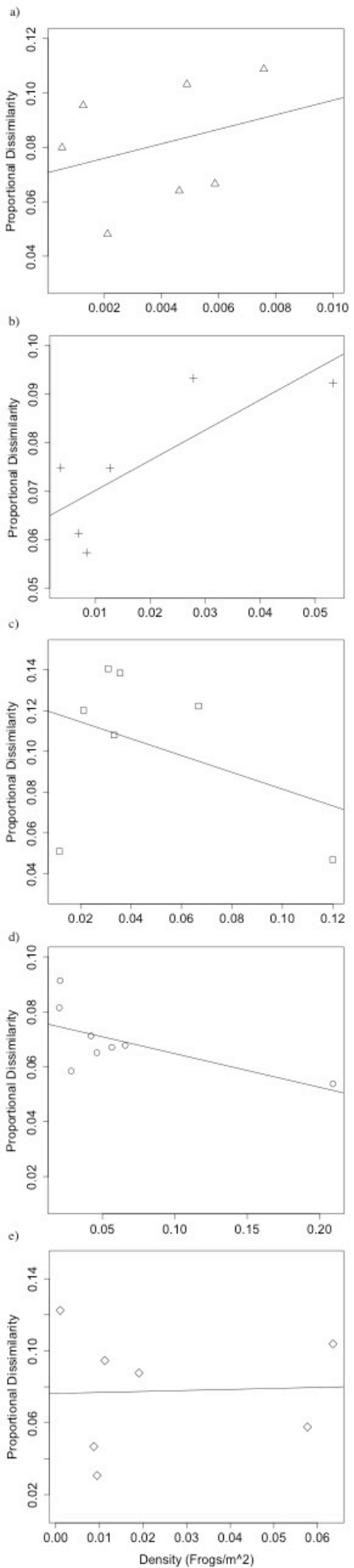
Figure 6
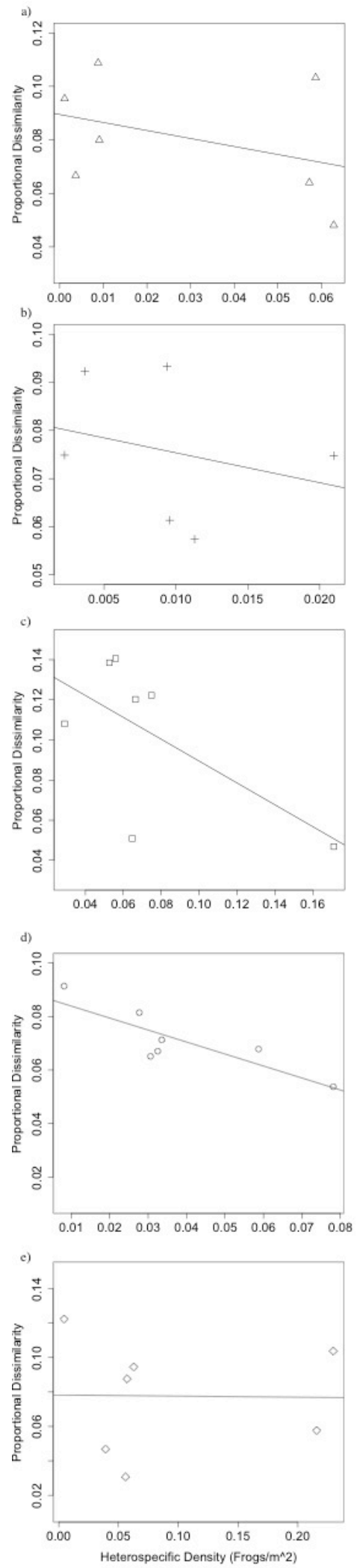
Figure 7

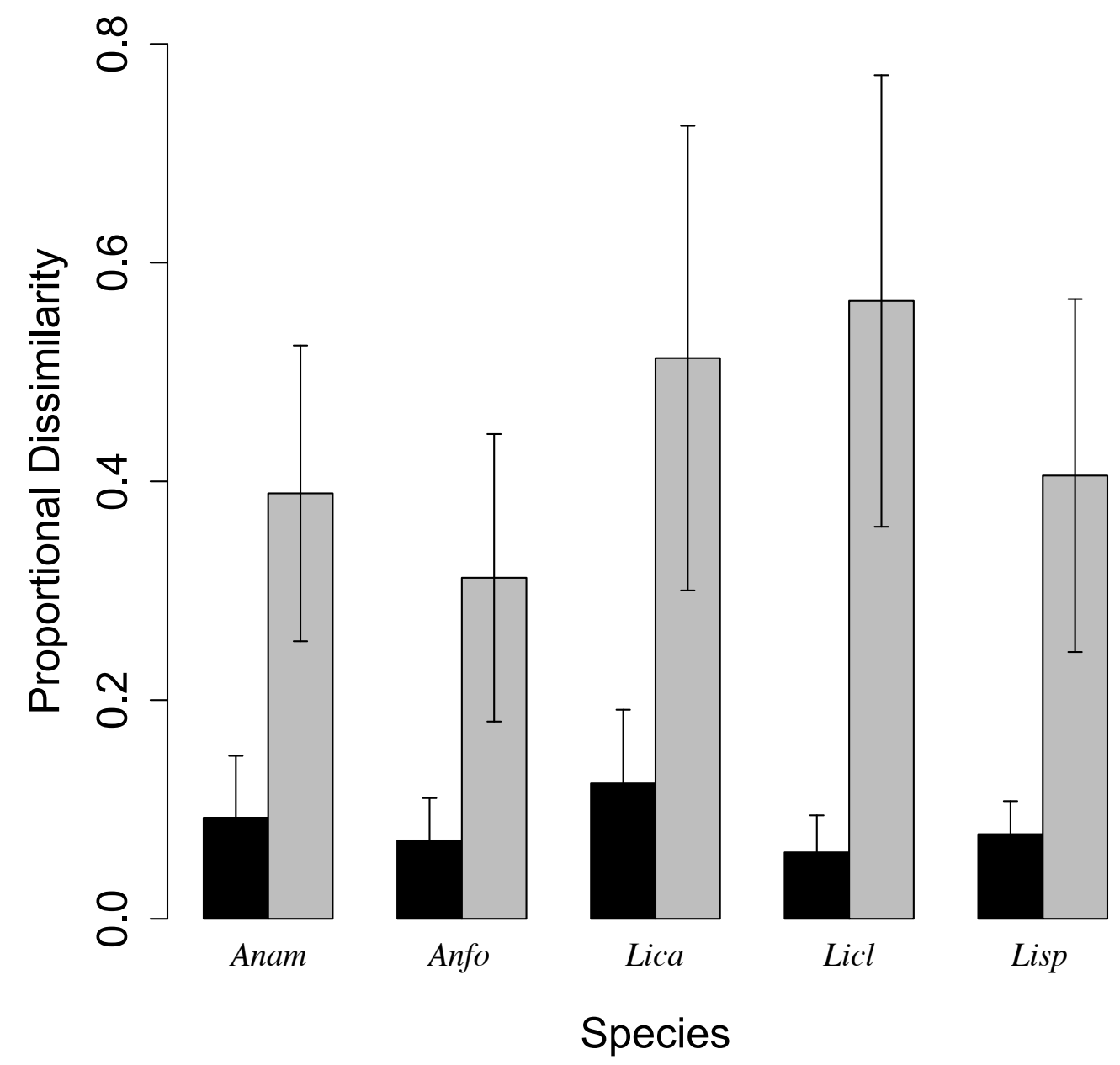




\title{
CHAPTER V
}

NICHE PARTITIONING AND THE ROLE OF INTRAPOPULATION NICHE VARIATION IN STRUCTURING A GUILD OF GENERALIST ANURANS

\begin{abstract}
SUMMARY
We investigated niche partitioning in five species of frogs and toads: Anaxyrus americanus, A.fowleri, Lithobates catesbeianus, L. clamitans, and L. sphenocephalus. We used stable isotopes and stomach contents to measure diets of the five species. We assessed differences in snout-vent length and examined whether those differences were related to diet variation. Additionally, we determined if species differed in their habitat use by measuring the distance between the locations of frogs and toads and the pond edge. We found that species differed significantly in isotope values. Values of $\delta^{15} \mathrm{~N}$ indicated that L.catesbeianus foraged at the highest trophic level of the considered species, and A.fowleri foraged at the lowest. Anaxyrus americanus, L. clamitans, and $L$. sphenocephalus had intermediate $\delta^{15} \mathrm{~N}$ values. Within most species, larger individuals had higher $\delta^{15} \mathrm{~N}$ values. Thus the $\delta^{15} \mathrm{~N}$ values of longer $A$. americanus and L. clamtians individuals tended to overlap those of shorter L. catesbeianus. Lithobates sphenocephalus followed the same pattern at several sites. The $\delta^{15} \mathrm{~N}$ values of shorter A. americanus and L. clamitans individuals tended to overlap those of smallest species, A.fowleri. Values of $\delta^{13} \mathrm{C}$ indicated that A.fowleri, A. americanus, and L. sphenocephalus foraged in primarily
\end{abstract}


terrestrial habitats while L. catesbeianus and L. clamitans foraged closer to ponds and on more aquatic prey. Our five species also differed in the prey that was found in their stomachs. Both A.fowleri and A. americanus took significantly more ants than the other species, and L. sphenocephalus took significantly more orthopterans than other species. All species preyed upon beetles more than would be expected based on environmental availability, and all species took non-flying prey in greater proportion than flying prey. Our results demonstrate the importance of considering individual-level variation when investigating niche partitioning, as such variation can affect the degree of overlap among species.

\section{INTRODUCTION}

Ecologically similar, sympatric species must partition their niches to avoid competitive exclusion (MacArthur 1958; Hardin 1960; Toft 1985; Siepielski and McPeek 2010). Niche partitioning has taken a central role in many population and community ecology studies, as it resolves the paradox between early theoretical/laboratory studies demonstrating competitive exclusion and the fact that many ecosystems have ecologically similar species that do not drive one another extinct (Gause 1932; Hutchinson 1959, 1961). Many fundamental ecological studies investigated how similar species can coexist (Homage to Santa Rosalia and the Hutchinsonian niche; Hutchinson 1959, 1957), how such species can divide seemingly homogenous resources (MacArthur's Warblers and the paradox of the plankton; MacArthur 1958, Hutchinson 1961), and how similar two species can be before one starts to exclude the other (Limiting similarity; MacArthur and 
Levins 1967). Thus, niche partitioning has been a central idea in ecology for over half a century.

Understanding how species partition their niche remains an active and important part of ecological research (Chesson 2000, Martin and Martin 2001, Siepielski and McPeek 2010, Correa and Winemiller 2014). Species can partition their resources a variety of ways. In many traditional studies on resource partitioning, researchers consider how environmental heterogeneity can enable species to differentially access resources. Species can feed in different habitats and microhabitats (Navarro et al. 2009; Symes et al. 2013; Cloyed 2014), on different items (Pulliam 1985; Beaulieu and Sockman 2012), at different times (Chesson 2000; Reum \& Essington 2008), and by using different foraging methods (Toft 1980; Frere et al. 2008; Knickle and Rose 2014; Newell et al. 2014; O'Neill and Gibb 2014). While many of these studies demonstrate niche partitioning, they do not consider the role that individual and/or ontogenic trait variation may play in niche partitioning (Hirai and Matsui 2002, Lasky et al. 2014).

Recent studies on generalist foragers have demonstrated that individuals of some of these species act as specialists and only take a subset of the species' resource spectrum (Bolnick et al. 2003, Wennersten and Forsman 2012). These intraspecific trait variations can take many forms (Bolnick et al. 2003), including between sexes (Meik et al. 2012; Grant et al. 2014; Rossman et al. 2015), individuals (Araújo et al. 2007; Tinker et al. 2008; Agashe and Bolnick 2010; Wennersten and Forsman 2012), and body sizes in species that are indeterminate growers and have size-structured populations (Hirai and Matsui 2002; Rudolf and Rasmussen 2013; Ortíz-Serrato et al. 2014; Zhao et al. 2014; Nifong et al. 2015). Variations of these types have important ecological and evolutionary 
consequences (Bolnick et al. 2011). For example, intraspecific trait variations can increase population stability through the portfolio effect (Schindler et al. 2010) or through reduced intraspecific competition (Svanbäck and Bolnick 2007; Tinker et al. 2008). Individual trait variation may promote species coexistence if it decreases the number of individuals from each species that are actually overlapping in niche space (Lichstein et al. 2007; Lasky et al. 2014). Furthermore, considering individual trait variation in studies of niche partitioning may help clarify some results in which it is hard to detect species differences. Trait variation at the individual level can reduce intraspecific competition, deteriorating niche boundaries and allowing for more overlap among species (Bolnick et al. 2011; Paine et al. 2011; Siefert 2012; Lasky et al. 2014).

In this study we investigated resource partitioning in five frog and toad species, Anaxyrus americanus, A.fowleri, Lithobates catesbeianus, L. clamitans, and L. sphenocephalus. These frogs and toads represent an excellent system to test resource partitioning and the role of intrapopulation trait variation has on species coexistence for two reasons. First, older studies on the diets of these species have shown that they have large dietary overlap (Hamilton, Jr. 1948; Hamilton, Jr. 1954; Bush and Menhinick 1962; McKamie and Heidt 1974). Although competition has not been studied across all five of our study species, some research has adressed competition between species pairs within this group. Lithobates catesbeianus is more likely to be found in ponds and to take a greater proportion of aquatic prey than L. clamitans (Werner et al. 1995). Additionally, L. catesbeianus is a superior competitor to L. clamitans (Hecnar and M'Closkey 1997), but a laboratory study demonstrated that L. clamitans is competitively superior to $A$. americanus (Sams and Boone 2010). Second, these species of frogs and toads are 
generalist foragers (Hamilton, Jr. 1948; Hamilton, Jr. 1954; Bush and Menhinick 1962; McKamie and Heidt 1974) and therefore offer an opportunity to investigate the role intrapopulation niche variation has on aiding niche partitioning. Studies on generalist frogs have found evidence of intrapopulation niche variation in frog species (Araújo et al. 2009; Benard and Maher 2011). Several species of frogs, including L. catesbeianus, increase their trophic level with increasing SVL (Harai and Matsui 2002; Ortíz-Serrato et al. 2014).

We used a multi-faceted approached to determine niche partitioning and to investigate the possible role intra-population niche variation has on this partitioning. To measure diets, we used both stable isotope analysis (SIA) and stomach content analysis. Additionally, we measured morphological traits to test whether diet patterns correlated with morphology, and we measured how far frogs and toads were found from the pond edge to measure spatial partitioning. We used the morphological variables to determine if individual patterns varied in a manner that would be conducive to species coexistence.

\section{MATERIALS AND METHODS}

Study Site: This study was performed at ten small ponds in Bernheim Arboretum and Research Forest, Clermont, Kentucky, U.S.A. Bernheim is located in the Knobs region of Kentucky. Ponds varied from $41 \mathrm{~m}^{2}$ to $1329 \mathrm{~m}^{2}$ in size. Eight ponds were in the forest interior and two were on the edge of a forest and small grassland. White oak-black oak (Quercus alba-Q.velutina) and white oak-chestnut oak (Q.alba-Q.prinus) were the most 
common forest types in Bernheim. The most abundant understory plants were Smilax spp., Toxicodendron radicans, Leersia oryzoides, and Microstegium vimineum.

Frog Capture and Processing: We collected frogs and toads from April through September in 2011 and 2012 and from April-June in 2013 and 2014. We captured frogs and toads with hand nets in and around ponds starting 30 minutes after sunset and continuing for an average of 55 minutes. When frogs and toads were captured, their initial location was marked and they were placed in plastic containers. Each evening after frogs and toads were collected, we measured the distance from their initial locations to the pond edge. When frogs were initially in the pond, we recorded the distance as a negative number.

We measured morphological variables with a Swiss Precision Instruments dial caliper to the nearest millimeter. To test whether morphology was correlated with diet, we measured morphological variables relating to feeding: gape width, mandible length, and head depth (Benard and Maher 2011). We also measured morphological variables related to jumping: snout-vent length (SVL), the lengths of the right femur, tibia, and the combined length of the metatarsals and fourth phalange (Nauwelaerts et al. 2007). We additionally created the variable leg length by summing the lengths of the femur, tibia, and meta-tarsal/phalanges.

We obtained stomach contents with a gentle stomach flushing technique (Solé et al. 2005). We caught any expelled stomach contents in a cup and stored them in $95 \%$ ethanol. The prey present were identified later in the laboratory. Prey groups for stomach 
content analysis included orthopterans, ants, coleopterans, miscellaneous flying prey, and miscellaneous non-flying prey. Any insect in the taxa Orthoptera, Coleoptera, or Formicidae (ants) was placed in its taxonomic group. Miscellaneous non-flying prey included spiders and insects from the families Pentatomidae, Reduviidae, and Membracidae, as well as other non-flying Hemipterans and larval lepidopterans. The miscellaneous flying prey group included flying Hymenoptera, most insects in Diptera, flying Hemipterans such as Cicadellidae, Cercopidae, adult lepidopterans, and adult odonates. We divided stomach contents into these prey groups because of their frequency in certain species stomachs.

Frogs and toads were individually marked with a unique combination of toe clips. Skin from these toe clips was used for SIA. The clipped toes were placed in a chilled cooler in the field, dried for 48 hours at $60^{\circ} \mathrm{C}$, and then stored in a cool, dark drawer in the laboratory. After the toe clip, frogs were released at the site where they were captured. All frogs were captured and handled under the approval of the Insitutional Animal Care and Use Committee at the University of Louisville (UL-IACUC-10037 and UL-IACUC-13026).

Stable Isotope Analysis: After samples were dried, we manually separated the bone of the toe from the skin and ligaments with an $\mathrm{Xacto}^{\odot}$ knife. Each skin sample was weighed to between $0.3-0.7 \mathrm{mg}$ in tin capsules on a Mettler Toledo AG245 micro-scale. Samples were analyzed at the University of New Mexico's Center for Stable Isotopes. The samples were combusted in a Costech 4010 elemental analyzer (Costech, Valenicia, CA) 
coupled to a Thermo Scientific Delta V mass spectrometer (Thermo Scientific, Bremen, Germany).

Stable isotope values were expressed with the standard delta notation $(\delta)$ in parts per thousand (\%), where $\delta X=\left(R_{\text {sample }} / R_{\text {standard }}-1\right) * 1000$, where $X$ is $\delta^{13} C$ or $\delta^{15} N$ and $R_{\text {sample }}$ and $\mathrm{R}_{\text {standard }}$ are the molar ratios of $\mathrm{C}^{13} / \mathrm{C}^{12}$ and $\mathrm{N}^{15} / \mathrm{N}^{14}$ of the sample and the standard reference material. The reference material was Vienna-Pee Dee Belemnite for carbon and atmospheric $\mathrm{N}_{2}$ for nitrogen.

Prey Collection: To determine local abundances of prey species, we collected arthropods around a pond within 10 days of sampling frogs or toads at that pond. To collect grounddwelling arthropods, we placed pit-fall traps on a $2 \mathrm{~m}$ wide transect that began at the pond's edge and continued 130m into the surrounding habitat. Two pit-fall traps were placed 0-2m, 13-15m, 28-30m, 113-115m, and 128-130m from each pond; at each of these five sites on a transect, the two traps were placed on opposite sides of the transect line and 1-2m apart. All traps were left open for 48 hours, after which the contents of traps were collected and taken to the laboratory for identification. Within a few minutes after contents from pitfall traps were collected, we took sweep-net samples to collect flying arthropods and arthropods in foliage close to the ground. Sweep netting took place at $0 \mathrm{~m}, 15 \mathrm{~m}, 30 \mathrm{~m}, 115 \mathrm{~m}$, and $130 \mathrm{~m}$ and used 20 of C.S.C.'s sweep-steps at each location. Collected arthropods were taken to the laboratory for identification. We summed prey from all sweep-net and pitfall traps for each pond, calculated the proportion prey for each prey category, and used these proportions as estimates of the frequency of prey in the 
environment.

Statistical Analyses: We used principal components analysis (PCA) to organize morphological variation, and made size adjustments with the method advocated by Berner (2011). We used ANOVA or the Kruskal-Wallis test to determine differences among species in the first two axes produced in the PCA, SVL, and distance to pond. To test for pairwise differences between species in these traits and in distance to pond, we used Tukey’s Honest Significant Differences test when an ANOVA was used and pairwise Wilcoxon tests with Bonferroni-adjusted $p$ values when a Kruskal-Wallis test was used.

We performed a MANCOVA to determine differences in isotopic values among species, years, and sexes. We included the values for the first two axes from the sizeadjusted PCA and SVL in the analysis. We included SVL because frogs are known to change their diets as they grow larger (Hirai and Matsui 2002). To determine if stable isotope baseline values varied among sites, we included site in the initial model. If site was significant, then we nested further analyses by site. Non-significant factors were dropped from further analyses. We performed follow-up ANCOVAs for $\delta^{13} \mathrm{C}$ and $\delta^{15} \mathrm{~N}$. Differences between species pairs for each $\mathrm{C}$ and $\mathrm{N}$ isotopic values were then determined with Tukey's Honest Significant Differences test.

We performed ANOVAs for each prey group on the numbers of individuals captured to determine if there were differences in numbers of prey among ponds. We also calculated Chesson's alpha (Chesson 1983) on the prey groups for SCA. Chesson's alpha 
compares prey frequency in stomachs to prey frequency in the environment and is therefore a measure of selectivity (Chesson 1983). Chesson's alpha ranges from 0 to 1 , where 0 represents complete avoidance of a prey type and 1 represents complete preference for a prey type. Given that we had five prey groups, a Chesson's alpha of 0.2 for a particular prey group implies that the frequency of prey in stomach contents matches the frequency of the prey in the environment. We used a perMANOVA in the R package Vegan (Oksanen et al. 2013) to determine differences among species, site, and date. All non-significant factors were dropped. This perMANOVA was done with 50,000 permutations using the Bray distance measure. We followed this perMANOVA with individual permutation tests between species to determine which species were significantly different from each other. These tests were done with 50,000 permutations using Markov chain Monte Carlo methods. All statistical analyses were done in R (R Core Team 2013).

\section{RESULTS}

Morphology and Distance to Pond: We performed PCA on 54 A. americanus, 37 A. fowleri, 68 L. catesbeiuanus, 75 L. clamitans, and 38 L. sphenocephalus. In the PCA, the first axis explained $72 \%$ of the morphological variation and loaded most strongly with leg length (0.48), while the second axis explained $20 \%$ of the variation and loaded mostly strongly with gape width (0.86). There were significant overall differences among species in the first PCA axis, associated with size-adjusted leg length (ANOVA: F=102.2, $\mathrm{df}=4,256, p<0.001)$. We used a Tukey's Honest Significant Differences test to determine 
pair-wise differences between each of the species. These differences were significant between L. clamitans and three other species, including A. americanus $(p<0.001), A$. fowleri $(p<0.001)$, and L. catesbeianus $(p<0.001)$. There were also significant differences in size-adjusted gape width between L. sphenocephalus and A. americanus $(p<0.001), A$. fowleri $(p<0.001)$, and L. catesbeianus $(p<0.001)$. There were also significant overall differences among species in the second PCA axis $(\mathrm{F}=54.81, \mathrm{df}=4,256, p<0.001)$. Lithobates sphenocephalus differed significantly from all four other species $(p<0.001$ for each comparison), but there were no significant differences between any other species pair.

Species differed significantly in SVL (ANOVA: Fig. 1a; $F=119.3$, df=4,237, $p<0.001)$. Tukey's Honest Significant Differences test showed that L. catesbeianus was significantly longer than the other four species (L. clamitans: $p<0.001 ; L$. sphenocephalus: $p<0.001 ;$ A. anaxyrus: $p<0.001$; and A.fowleri: $p<0.001)$. In addition, L. clamitans was significantly longer than L. sphenocephalus ( $p=0.003)$, A. anaxyrus ( $\mathrm{p}<0.001)$, and A.fowleri $(p<0.001)$, and L. sphenocephalus was significantly longer than the two toads (A. americanus: $p=0.0532$; and A. fowleri: $p=0.0126)$.

Distance to pond edge varied significantly among species (Fig. 1b; Kruskal-Wallis test: $\left.\mathrm{X}^{2}=212.18, \mathrm{df}=4, p<0.001\right)$. Pairwise Wilcoxon tests showed that $A$.fowleri toads were found at significantly greater distances from ponds than were members of the other species (A. americanus: $p=0.001 ;$ L. sphenocephalus: $p<0.001 ;$ L. catesbeianus: $p<0.001$; and L. clamitans: $p<0.001)$. Anaxyrus americanus toads were also found farther from ponds than were any of the frog species (L. sphenocephalus: $p<0.001$; L. catesbeianus: 
$p<0.001$; and L.clamitans: $p<0.001)$. Lithobates sphenocephalus occupied sites at greater distance from pond than either L. catesbeianus $(p<0.001)$ or L. clamitans $(p<0.001)$.

Stable Isotope Analysis: Isotopic values varied among sites $(\mathrm{Pillai}=0.4744, \mathrm{~F}=8.71, \mathrm{df}=$ $8,253, p<0.001)$, and we therefore nested further analyses by site. The MANCOVA indicated that species differed in $\mathrm{C}-\mathrm{N}$ isotopic space (Fig. 2; Pillai=0.719, $\mathrm{F}=35.53$, $\mathrm{df}=8,253, p<0.001)$ and that within species the body length of individuals influenced the isotopic space they occupied (Pillai $=0.259, \mathrm{~F}=44.06, \mathrm{df}=1,253, p<0.001$ ). The $\mathrm{C}$ and $\mathrm{N}$ isotopic ratios of the study species were not significantly affected by year (Pillai=0.0241, $\mathrm{F}=2.621, p=0.075$ ), sex (Pillai $=0.007, \mathrm{~F}=0.919, \mathrm{df}=2,249, p=0.4$ ), or the morphological traits that were incorporated in PCA axes one (Pillai $=0.016, \mathrm{~F}=1.963, p=0.143$ ) and two (Pillai $=0.008, \mathrm{~F}=0.372, p=0.372$ ). We therefore dropped these variables from further analyses.

Follow-up ANCOVAS for each isotope type revealed similar results. For nitrogen isotopes, species differed in isotopic space (Fig. 3a; $\mathrm{F}=55.87, \mathrm{df}=4,253, p<0.001$ ) and individuals with longer SVLs had higher nitrogen isotope values (Fig. 4a; F=57.49, $\mathrm{df}=1,253, p<0.001)$. Anaxyrus fowleri had the lowest nitrogen isotope values, indicating that this species was feeding at the lowest trophic level. Anaxyrus americanus, $L$. clamitans and L. sphenocephalus had intermediate nitrogen isotope values, indicating that these species feed at intermediate trophic levels. Lithobates catesbeianus had the highest nitrogen isotope values (Fig. 3a). For carbon isotopes, we found that each species had different isotopic spaces (Fig. 3b; $\mathrm{F}=64.52 ; \mathrm{df}=4,255, p<0.001$ ) and within each species 
individuals with longer SVL generally had higher carbon isotope values (Fig. 4b; $\mathrm{F}=38.56, \mathrm{df}=1,255, p<0.001)$. In general, A. americanus, $A$. fowleri and longer $L$. catesbeianus individuals had higher carbon isotope values and L. clamitans, L. sphenocephalus and smaller L. catesbeianus had lower carbon isotope values.

For each species, we used linear models nested by site to better understand the relationship between each isotope type and SVL. When accounting for differences in isotope values among sites, individuals of A. americanus, L. catesbeianus, and $L$. sphenocephalus with longer SVL had higher $\delta^{15} \mathrm{~N}$ values (Fig. 5a,c,d; A. americanus: $\mathrm{t}=$ 4.028, $\mathrm{df}=9,54, p<0.001 ;$ L. catesbeianus: $\mathrm{t}=8.3, \mathrm{df}=7,60, p<0.001 ;$ L. clamitans: $\mathrm{t}=2.708, \mathrm{df}=9,64, p=0.009)$. Individuals of A. americanus and Lithobates sphenocephalus that had longer SVL did not have higher $\delta^{15} \mathrm{~N}$ values (Fig. 5b,e; $A$. fowleri: $\mathrm{t}=0.158, \mathrm{df}=7,30, p=0.875 ;$ L. sphenocephalus: $\mathrm{t}=1.815, \mathrm{df}=9,29, p=0.0799)$. Individuals of both A. americanus and L. catesbeianus with longer SVL also had higher $\delta^{13} \mathrm{C}$ values (Fig. 5a,c; A. americanus: $\mathrm{t}=3.79, \mathrm{df}=9,44, p<0.001 ;$ L. catesbeianus: $\mathrm{t}=3.96$, $\mathrm{df}=7,60, p<0.001)$. Individuals of A.fowleri, L. clamitans, and L. sphenocephalus with longer SVL did not have significantly different $\delta^{13} \mathrm{C}$ values (Fig. 5b,d,e; A. fowleri: $\mathrm{t}=1.427, \mathrm{df}=7,30, p=0.164 ;$ L. clamitans: $\mathrm{t}=0.879, \mathrm{df}=9,64, p=0.383 ; L$. sphenocephalus: $\mathrm{t}=1.157, \mathrm{df}=9,29, p=0.257)$.

Prey Availability: We tested for differences among sites of each prey type using ANOVA. The numbers of orthopterans and ants captured in pit-fall traps and sweep-net samples differed significantly across sites (Table 1; orthopterans: $F=2.723$, $\mathrm{df}=8,43$, 
$p=0.009$; ants: $\mathrm{F}=2.7, \mathrm{df}=8,43, p=0.01)$. A Tukey honest significant differences test found that the numbers of orthopterans differed significantly only between Mud Hole and Tannin Pond North (Table $1 ; p=0.0191)$. Ants were significantly more/less abundant at Tannin Pond North than at Cattail Puddle (Table $1 ; p=0.007)$ or Mud Hole $(p=0.023)$ but did not otherwise vary significantly across sites. Beetles, miscellaneous flying prey, and miscellaneous non-flying prey did not differ significantly among sites (Table 1; beetles: $\mathrm{F}=0.981, \mathrm{df}=8,43, p=0.478$; miscellaneous flying prey: $\mathrm{F}=1.238, \mathrm{df}=8,43, p=0.292$; miscellaneous non-flying prey: $\mathrm{F}=1.72, \mathrm{df}=8,43, p=0.101)$.

Stomach Content Analysis: We obtained stomach contents from 36 A. americanus, 26 A. fowleri, 39 L. catesbeianus, 100 L. clamitans, and 30 L. sphenocephalus. The proportions of prey present in stomachs differed significantly among species (Fig. 5, Table 2; $\mathrm{F}=7.159, \mathrm{df}=4,146, p<0.001)$, sites $(\mathrm{F}=1.545, \mathrm{df}=8,146, p=0.026)$, and dates $(\mathrm{F}=1.5$, $\mathrm{df}=70,146, p<0.001)$. In general, both toad species preferred ants while the three frog species avoided them (Fig. 6; Table 2). L. sphenocephalus preferred orthopterans while the other species avoided them to varying degrees (Fig. 6; Table 2). However, all five species either preferred or took coleopterans in proportion to environmental abundances, preyed upon miscellaneous non-flying predators more than miscellaneous flying predators (Fig. 6), and preyed upon miscellaneous flying prey less than environmental abundances would predict. Since more ants were found in stomachs of both toad species, sites that had more toads compared to true frogs had more ants. Similarly, orthopterans were more common in stomachs at sites where more L. sphenocephalus individuals were present. Both toad species as well as L. sphenocephalus were more common in the spring 
than during the summer. Stomach contents obtained from early in the year had more ants and more orthopterans.

\section{DISCUSSION}

We examined isotopic niche, stomach contents, morphology, and habitat selection to determine how five species of frogs and toads coexist. These five anurans partitioned their resources and habitat in complex ways. Although any particular species differed from each of the others on at least one of these measures, no two species differed significantly from one another on all measures. We also found intrapopulation variation in niche that may influence interspecific competition.

Niche partitioning: Overall, species varied in trophic level, as indicated by $\delta^{15} \mathrm{~N}$ values. In general, larger species had higher $\delta^{15} \mathrm{~N}$ values, indicating that they occupied higher trophic positions (Post et al. 2002; Martínez del Rio et al. 2009). Lithobates catesbeianus, the species with the longest SVL, had the highest $\delta^{15} \mathrm{~N}$ values and foraged at the highest trophic level. A.fowleri, one of the two smallest species, had the lowest $\delta^{15} \mathrm{~N}$ values and thus foraged at the lowest trophic level. Anaxyrus americanus, L. clamitans, and L. sphenocephalus occupied similar, intermediate trophic positions (Fig. 3a), but they differed in other key ecological factors. Anaxyrus americanus had the smallest SVL (Fig. 1a), foraged farther from the ponds (Fig. 1b), and took significantly more ants (Fig. 6, Table 1) than did either L. clamitans or L. sphenocephalus. Lithobates sphenocephalus 
had a smaller SVL (Fig. 1a), was found farther from ponds (Fig. 1b), and took more orthopterans (Fig. 6, Table 1) than L. clamitans.

Our frog and toad species also differed significantly in $\delta^{13} \mathrm{C}$ values, which vary depending on the consumption of resources from aquatic (lower $\delta^{13} \mathrm{C}$ values) versus terrestrial (higher $\delta^{13} \mathrm{C}$ values) habitats (Newsome et al. 2007). Both toad species had high $\delta^{13} \mathrm{C}$ values (Fig. 3b), indicating that aquatic prey and habitats were not important foraging areas for these toads; these results are supported by our habitat selection data, which showed that both toad species were found relatively far from ponds (Fig. 1b), and by previous studies showing that toads did not visit ponds except for breeding (Rothermel and Semlitsch 2002; Forester et al.2006). Lithobates sphenocephalus was intermediate between the two toads and the other two frog species both in $\delta^{13} \mathrm{C}$ values (Fig. 3b), and in the distance from ponds at which they foraged (Fig. 1b). An earlier study similarly found that L. sphenocephalus often ventured into upland habitat and farther from water between breeding periods (Graeter et al. 2006). Lithobates catesbeianus frogs had the lowest $\delta^{13} \mathrm{C}$ values in our study and were found close to ponds and frequently in them. A previous study found that Lithobates catesbeianus preyed upon aquatic organisms more frequently than did L. clamitans (Werner et al. 1995), which could explain the lower $\delta^{13} \mathrm{C}$ values of L. catesbeianus. However, Werner et al. (1995) also found that L. catesbeianus individuals were located closer to bodies of water and were more often in water than were L. clamitans frogs. In contrast, we found no significant difference in distance to pond between the two species, and we found them in ponds at equal frequencies (Fig. 1b). 
Stomach contents help elucidate niche differences among these ecologically and evolutionarily related species. Ants formed a greater proportion of the diet in the two toad species than in any of the frog species (Fig. 6, Table 2), supporting older studies suggesting that toads in general often prey upon ants more than do most frog species (Toft 1980,1985). However, according to stomach contents, A. americanus is more of a generalist, while A.fowleri specializes on ants and coleopterans (Fig. 6, Table 2).

Lithobates sphenocephalus was the only species to take orthopterans in numbers greater than expected based on orthopteran abundance (Fig. 6, Table 2). Non-flying, miscellaneous prey are more frequent than flying, miscellaneous prey in the stomachs of all frogs and toads in our study (Fig. 6, Table 2), a bias that may result from a greater difficulty of capturing flying prey.

The significant variation we found in the stomach contents among sites and dates is likely attributable to site and seasonal variation in the frequency of each anuran species. Several sites consistently had relatively more A. americanus, A. fowleri, and $L$. sphenocephalus. At those sites, ants and orthopterans were more abundant in stomach contents because ants were frequent prey for the toad species and orthopterans were frequent prey for L. sphenocephalus. At other sites, where L. catesbeianus and $L$. clamitans were more abundant, beetles and other miscellaneous non-flying prey were more frequent as stomach contents. Additionally, both toad species and $L$. sphenocephalus were more likely to be captured in spring and early summer than the other two species, which were more frequently captured in mid to late summer. As a result, stomach contents from early in the field season were more likely to include ants 
and orthopterans, while stomach contents from later in the season were more likely to include beetles and miscellaneous non-flying prey.

In our system, stomach contents provided diet data slightly different from the results of the isotopic analyses. Stomach content analysis and stable isotope analysis each provided a unique perspective on anuran diets and, when combined, aided in understanding the multifaceted niche partitioning that can occur in natural populations. In some systems, researchers can compare the same prey groups between stomach contents and stable isotope analysis, and in those studies results from the two kinds of analyses are similar (Jansen et al. 2013; Ruiz-Cooley et al. 2006). However, such direct comparisons are often not possible, and consequently many researchers have similarly found that the two-pronged approach using both kinds of analysis provides two different perspectives into diet and or habitat use (e.g. Polo-Silva et al. 2013; Bosley et al. 2014). Stomach content analysis can provide not only short term diet information (Kolts et al. 2013; Connan et al. 2014), but also much more detailed taxonomic information about prey (Kolts et al. 2013; Polo-Silva et al. 2013; Bosley et al. 2014). Stable isotopes can help determine trophic structures (Post 2002) and define sources of carbon when habitats and diets vary in their $\delta^{13} \mathrm{C}$ values (Newsome et al. 2007).

Intraspecific niche variation: The relationships between isotopic niche and SVL that we observed across species also occurred within species. Within most species in our study, larger individuals had higher $\delta^{15} \mathrm{~N}$ values (Fig. 5a-e): within sites there was a significant positive relationship between $\delta^{15} \mathrm{~N}$ values and SVL for A. americanus, L. clamitans, and 
L. catesbeianus. Longer A. americanus and L. clamitans individuals had $\delta^{15} \mathrm{~N}$ values similar to those of shorter L. catesbeianus individuals (Fig. 5a, c, d). The $\delta^{15} \mathrm{~N}$ values of longer A. americanus and L. clamitans overlapped with those of shorter L.catesbeianus individuals (Fig. 5c, d, e). Shorter individuals of both A. americanus and L.clamitans overlapped more with A. fowleri individuals, which were overall the shortest species. At some sites, $\delta^{15} \mathrm{~N}$ values of longer L. sphenocephalus overlapped those of shorter bodied L. catesbeianus individuals (Fig. 5c, e). Lithobates sphenocephalus had the smallest sample size and was the most infrequent frog found at our study sites. The relationship of body size and trophic level in L. spehnocephalus may be more pronounced with a greater sample size.

This intrapopulation niche variation caused by size can potentially alter competitive interactions and affect niche partitioning (Lichstein et al. 2007; Bolnick et al. 2011; Lasky et al. 2014). In indeterminate growers such as anurans, the variation in body size across the ontogeny of the individual may contribute to intrapopulation niche variation, as in age-structure populations there will be individuals of many sizes (Hasumi et al. 2010; Cogălnicean et al. 2013). In the case of L.catesbeianus, during the early part of the adult phase of their life history they will overlap in trophic level with several of the other species of frogs and toads, but once they reach $\sim 120 \mathrm{~mm} \mathrm{SVL}$, they begin to occupy a higher trophic level than the other species and are freed from trophic overlap with the other study species (Fig. 5c). For the other species, many of them will change competitive interactions across the adult phase of their life history, such that early in their lives they will not be in competition with the same species as later in their life. Since only a proportion of the population is therefore competing with heterospecifics, the interaction 
strength of that competition is reduced and this can aid niche partitioning. Furthermore, body size has important effects on diet and many indeterminate growers vary their diet across their lives (Hirai and Matsui 2002; Hampton 2011; Heupel et al. 2014; OrtízSerrato et al.2014). Other studies of diet in anurans have demonstrated that overlap among species depends on body size and that changes in body size through ontogeny can change competitive interactions (Werner 1994; Werner et al. 1995; Hirai and Matsui 2002; Benard and Maher 2011; Ortíz-Serrato et al. 2014). Between L. catesbeianus and L. clamitans competition changes throughout the life cycle. Individuals of the two species that are similar in size at metamorphosis have greater diet overlap, but as L. catesbeianus becomes larger than L. clamitans their diets diverge (Werner et al. 1995). While little work has studied how intrapopulation niche variation can influence species coexistence, this line of research should be investigated in a large range of taxa to test for its generality.

The relationship between individual size and trophic level in these frogs and toads is not as simple as it might seem. The immediate explanation would be that as individuals become longer they eat larger prey and those larger prey should be higher on the trophic level. For many vertebrate taxa, individuals and species that are larger are assumed to feed at higher trophic levels because they feed on larger prey (Brose et al. 2005). Larger prey are assumed to be at higher trophic levels than smaller prey. However, amongst the arthropods that comprise the diets of the majority of our frogs and toads, there is no clear relationship between trophic level and body size (Brose et al. 2005). Indeed, many of the largest arthropods in our study sites were orthopterans, which are primary consumers 
(Cloyed, personal observations). This suggests that larger individuals may not merely be selecting larger prey but may be selecting prey that are themselves predators.

Intrapopulation niche variation associated with individual size may result from both ontogenetic change and individual variation. While frogs are indeterminate growers and longer individuals are often older, tadpole development and size at metamorphosis will also determine how long an individual becomes (Collins 1979; Ficetola and Bernardi 2006). Faster-developing tadpoles may be shorter at metamorphosis and subsequently be shorter throughout their mature stages of life (Ficetola and Bernardi 2006). As a result, the intrapopulation variation in size in our study species may be caused by both the effects of ontogeny in indeterminate growers and inter-individual morphological differences associated with tadpole development and metamorphosis. This may be especially true of L. catesbeianus, a species in which individuals that overwinter as tadpoles have very large body sizes at metamorphosis (Boone et al. 2004; Cloyed, personal observation). However, better studies are needed to understand the link between tadpole development and size at metamorphosis and how size at metamorphosis may generate variation in the diets of adult frogs and toads.

Values of $\delta^{13} \mathrm{C}$ also varied with size within A. americanus and L. catesbeianus. In general, larger individuals of both of these species had higher $\delta^{13} \mathrm{C}$ values (Fig. 5a, c). In the smaller individuals, a small amount of the isotopes in their skin may have remained from the aquatic tadpole phase, as the half-life of carbon isotopes in frog skin is about 90 days (Cloyed et al. in review). In larger individuals, any isotopic memory from the tadpole stage or the post-metamorphic stage will have been lost. Indeed, others have found ontogenetic shifts in diet between tadpoles and frogs using stable isotopes 
(Jefferson and Russell 2008; Trakimas et al. 2011; Huckembeck et al. 2014). While $L$. sphenocephalus did not overall have significantly higher $\delta^{13} \mathrm{C}$ values with larger SVL, there was an positive trend at several sites (Fig. 5e), indicating that they too moved away from aquatic habitats as they grew larger. Lithobates clamitans, on the other hand, did not show any overall trends between SVL and $\delta^{13} \mathrm{C}$ values (Fig. 5d), and L. clamitans may continue to use more aquatic habitats and prey as they grow larger. Stomach contents help illuminate niche differences among these ecologically and evolutionarily related species. As mentioned above, both toad species take ants in much greater proportion than any of the frog species (Fig. 6, Table 2). Toads frequently prey upon ants more than most frog species (Toft 1980, 1985). However, A. americanus is more a generalist according to stomach contents, while A. fowleri specializes on ants and coleopterans (Fig. 6, Table 2). Lithobates sphenocephalus was the only species to take orthopterans in great numbers (Fig. 6, Table 2). Non-flying, miscellaneous prey are more frequent than flying, miscellaneous prey in the stomachs of all frogs and toads in our study (Fig. 6, Table 2). This bias towards non-flying prey may result from difficultly in capturing flying prey.

Variation in the stomach contents among sites and across dates is likely attributable to the frequency of each species at different sites and at different times of the season. Several sites had relatively more A. americanus, A. fowleri, and $L$. sphenocephalus and relatively fewer $L$. catesbeianus and $L$. clamitans. In stomachs at those sites, ants and orthopterans were more common because ants were a common prey type of both toad species and orthopterans were a common prey type of $L$. sphenocephalus. At sites where L. catesbeianus and L.clamitans are more common, beetles and other miscellaneous non-flying prey were more common stomach contents. 
Additionally, both toad species were more likely to be encountered earlier in the season, as was L. sphenocephalus. Stomachs examined earlier in the season are likely to have more orthopterans and ants compared to stomachs examined later in the season, which are more likely to have beetles and other miscellaneous non-flying prey.

In our system, stomach contents provide diet data slightly differently than the isotope data does. We found that stomach content analysis and stable isotope analysis each provided unique perspectives of anuran diets and, when combined, aided in understanding the multifaceted resource partitioning that can occur in natural populations. In other studies on diet that have combined stable isotope and stomach content data, many have found that the two-pronged approach provide two different perspectives into diet and or habitat use (Polo-Silva et al. 2013; Bosley et al. 2014). In many of these studies, stomach content analysis provides not only short term diet information (Kolts et al. 2013; Connan et al. 2014), but can provide much more detailed taxonomic information (Kolts et al. 2013; Polo-Silva et al. 2013; Bosley et al. 2014). Stable isotopes can help inform trophic structures (Post 2002) and sources of carbon when habitats and diets vary in their $\delta^{13} \mathrm{C}$ values (Newsome et al. 2007). Other systems have the ability to compare the same prey groups between stomach contents and stable isotope analysis (Jansen et al. 2013; Ruiz-Cooley et al. 2006). 


\section{TABLES}

Table 1: Mean proportion $( \pm \mathrm{SD})$ of each prey group by site.

\begin{tabular}{cccccc}
\hline Site & Orthoptera & Beetles & Ants & Misc. Flying & Misc. Non-Flying \\
\hline Back-of-Nowhere & $0.11( \pm 0.07)$ & $0.18( \pm 0.17)$ & $0.14( \pm 0.05)$ & $0.27( \pm 0.11)$ & $0.30( \pm 0.09)$ \\
Cattail Puddle & $0.08( \pm 0.04)$ & $0.07( \pm 0.04)$ & $0.06( \pm 0.06)$ & $0.43( \pm 0.14)$ & $0.37( \pm 0.12)$ \\
Honey Hole & $0.08( \pm 0.08)$ & $0.13( \pm 0.04)$ & $0.07( \pm 0.04)$ & $0.25( \pm 0.19)$ & $0.47( \pm 0.19)$ \\
Mud Pond & $0.13( \pm 0.11)$ & $0.11( \pm 0.10)$ & $0.08( \pm 0.13)$ & $0.35( \pm 0.08)$ & $0.33( \pm 0.17)$ \\
Platform Pond & $0.13( \pm 0.03)$ & $0.09( \pm 0.04)$ & $0.20( \pm 0.08)$ & $0.25( \pm 0.07)$ & $0.33( \pm 0.11)$ \\
Tannin Pond North & $0.01( \pm 0.02)$ & $0.17( \pm 0.04)$ & $0.29( \pm 0.11)$ & $0.29( \pm 0.08)$ & $0.23( \pm 0.07)$ \\
Tannin Pond South & $0.06( \pm 0.03)$ & $0.10( \pm 0.05)$ & $0.19( \pm 0.12)$ & $0.33( \pm 0.14)$ & $0.33( \pm 0.15)$ \\
Vernal Pool & $0.14( \pm 0.10)$ & $0.08( \pm 0.08)$ & $0.12( \pm 0.06)$ & $0.21( \pm 0.12)$ & $0.45( \pm 0.06)$ \\
Wilson Road North & $0.08( \pm 0.06)$ & $0.13( \pm 0.07)$ & $0.15( \pm 0.10)$ & $0.28( \pm 0.12)$ & $0.36( \pm 0.09)$
\end{tabular}


Table 2: Results from between species permutation tests of Chesson's alpha selectivity index. Probability values have been Bonferroni adjusted. Negative t-values indicate that the latter species in the listed pair take less than the former species. Anam=Anaxyrus americanus, $\mathrm{Anfo}=$ A. fowleri, Lica $=$ Lithobates catesbeianus, Licl= L. clamitans, $\mathrm{Lisp}=$ L. sphenocephalus.

\begin{tabular}{|c|c|c|c|c|c|}
\hline $\begin{array}{l}\text { Species } \\
\text { Interaction }\end{array}$ & Orthoptera & Coleoptera & Ants & Misc. Flying & $\begin{array}{l}\text { Misc. Non- } \\
\text { Flying }\end{array}$ \\
\hline \multirow[t]{2}{*}{ Anam-Anfo } & $\mathrm{t}=-0.350$ & $\mathbf{t}=\mathbf{2 . 3 0 7}$ & $\mathrm{t}=-0.344$ & $t=-3.762$ & $t=-5.027$ \\
\hline & $p=0.363$ & $p=0.007$ & $p=0.326$ & $p<0.001$ & $p=<0.001$ \\
\hline \multirow[t]{2}{*}{ Anam-Lica } & $\mathrm{t}=0.0021$ & $\mathrm{t}=0.001$ & $t=-14.03$ & $\mathrm{t}=\mathbf{2 . 9}$ & $t=1.96$ \\
\hline & $p=0.494$ & $p=0.495$ & $p<0.001$ & $p=0.001$ & $p=0.019$ \\
\hline \multirow[t]{2}{*}{ Anam-Licl } & $t=3.028$ & $t=2.371$ & $t=-10.79$ & $\mathrm{t}=3.226$ & $\mathrm{t}=1.336$ \\
\hline & $p=0.001$ & $p=0.006$ & $p<0.001$ & $P<0.001$ & $p=0.077$ \\
\hline \multirow[t]{2}{*}{ Anam-Lisp } & $\mathrm{t}=3.552$ & $\mathrm{t}=-0.506$ & $t=-5.968$ & $\mathrm{t}=-0.42$ & $\mathrm{t}=1.511$ \\
\hline & $P<0.001$ & $p=0.315$ & $p<0.001$ & $p=0.346$ & $p=0.056$ \\
\hline \multirow[t]{2}{*}{ Anfo-Lica } & $\mathrm{t}=0.326$ & $\mathrm{t}=-1.079$ & $t=-13.15$ & $\mathrm{t}=\mathbf{2 . 7 4 2}$ & $\mathrm{t}=4.451$ \\
\hline & $p=0.375$ & $p=0.143$ & $p<0.001$ & $p=0.002$ & $p<0.001$ \\
\hline \multirow[t]{2}{*}{ Anfo-Licl } & $t=3.405$ & $t=-2.031$ & $t=-9.923$ & $t=4.619$ & $\mathrm{t}=4.117$ \\
\hline & $p<0.001$ & $p=0.028$ & $p<0.001$ & $p<0.001$ & $p<0.001$ \\
\hline \multirow[t]{2}{*}{ Anfo-Lisp } & $\mathbf{t}=\mathbf{3 . 7 0 7}$ & $t=-3.27$ & $t=-5.522$ & $\mathrm{t}=1.244$ & $t=2.739$ \\
\hline & $p<0.001$ & $p=0.002$ & $p<0.001$ & $p=0.098$ & $p=0.002$ \\
\hline \multirow[t]{2}{*}{ Lica-Licl } & $t=3.088$ & $t=-0167$ & $\mathrm{t}=2.997$ & $\mathrm{t}=-0.272$ & $\mathrm{t}=-\mathbf{5 . 8 0 3}$ \\
\hline & $p<0.001$ & $p=0.458$ & $p=0.001$ & $p=0.417$ & $p<0.001$ \\
\hline \multirow[t]{2}{*}{ Lica-Lisp } & $\mathrm{t}=3.577$ & $t=-2.1$ & $\mathrm{t}=1.159$ & $t=-4.597$ & $\mathrm{t}=-1.642$ \\
\hline & $p<0.001$ & $p=0.025$ & $p=0.116$ & $p<0.001$ & $p=0.06$ \\
\hline \multirow[t]{2}{*}{ Licl-Lisp } & $\mathrm{t}=\mathbf{2 . 3 0 7}$ & $t=-1.995$ & $\mathrm{t}=-0.392$ & $t=-4.271$ & $\mathrm{t}=0.922$ \\
\hline & $p=0.007$ & $p=0.038$ & $p=0.368$ & $p<0.001$ & $p=0.159$ \\
\hline
\end{tabular}




\section{FIGURE LEGENDS}

Figure $1-$ a) Snout-vent length (mm) by species. Anaxyrus americanus and A.fowleri were the smallest species, followed by Lithobates sphenocephalus and L.clamitans. Lithobates catesbeianus was the largest species but also had the largest variance, with individuals falling within the body size range of all other species. b) Distance to pond (m) by species. Both toad species are infrequently found near ponds and A.fowleri generally are found further from ponds than A. americanus. Lithobates clamitans and $L$. catesbeianus have about an equal distance to pond, with both species frequently found in the pond (negative numbers). Lithobates sphenocephalus is found further from ponds than the other two frog species but closer than both toad species.

Figure $2-$ Stable isotope biplot with mean $( \pm \mathrm{SE})$ of each species. Higher $\delta^{13} \mathrm{C}$ values indicate more terrestrial diet and lower $\delta^{13} \mathrm{C}$ values indicate more aquatic diet. Red circle = Anaxyrus americanus; orange triangle $=$ A. fowleri; purple cross $=$ Lithobates catesbeianus; green asterisk $=$ L. clamitans; blue diamond $=$ L. sphenocephalus .

Figure $3-$ a) $\delta^{15} \mathrm{~N}$ values by species. Anaxyrus americanus, Lithobates clamitans, and $L$. sphenocephalus have similar $\delta^{15} \mathrm{~N}$ values while L. catesbeianus has higher $\delta^{15} \mathrm{~N}$ values and $A$. fowleri has lower values. b) $\delta^{13} \mathrm{C}$ values by species. Anaxyrus americanus and $A$. fowleri have similar $\delta^{13} \mathrm{C}$ values; L. clamitans and L. sphenocephalus have similar $\delta^{13} \mathrm{C}$ values; L. catesbeianus has the lowest $\delta^{13} \mathrm{C}$ values but also has the greatest variance and overlaps with all other species. 
Figure $4-a) \delta^{15} \mathrm{~N}$ values by Snout-Vent Length (SVL). Higher $\delta^{15} \mathrm{~N}$ indicates higher trophic levels. Red circle $=$ Anaxyrus americanus; orange triangle $=$ A. fowleri; purple cross $=$ Lithobates catesbeianus; green asterisk $=$ L. clamitans; blue diamond $=L$. sphenocephalus. b) $\delta^{13} \mathrm{C}$ values by SVL. Higher $\delta^{13} \mathrm{C}$ values indicate a diet based on more terrestrial resources and lower $\delta^{13} \mathrm{C}$ values indicate diet with a larger aquatic component.

Figure $5-\delta^{15} \mathrm{~N}$ values (top row) and $\delta^{13} \mathrm{C}$ values (bottom row) versus snout-vent length (SVL) for each species: a) Anaxyrus americanus, b) A.fowleri, c) Lithobates catesbeianus, d) L. clamitans, e) L. sphenocephalus. Open box = Back-of-Nowhere, open circle $=$ Cattail Puddle, right-side up triangle $=$ Wilson Road North, cross $=$ Wilson Road South, open diamond = Platform Pond, open box with an $\mathrm{x}=$ Tannin Pond North, upsidedown triangle $=$ Tannin Pond South, closed box $=$ Honeyhole, closed circle $=$ Mudhole closed triangle $=$ Vernal Pool

Figure 6-Mean Chesson's alpha for each prey group on all species. All species prey upon beetles about equally and all species prey upon miscellaneous non-flying prey than miscellaneous flying prey. The two toad species, Anaxyrus americanus and A.fowleri, prey upon ants the most. Lithobates sphenocephalus prey upon orthopterans more than the rest of the other species. Red $=$ A. americanus; orange $=$ A. fowleri; purple $=L$. catesbeianus; green $=$ L. clamitans; blue $=$ L. sphenocephalus. 
Figure 1

a)

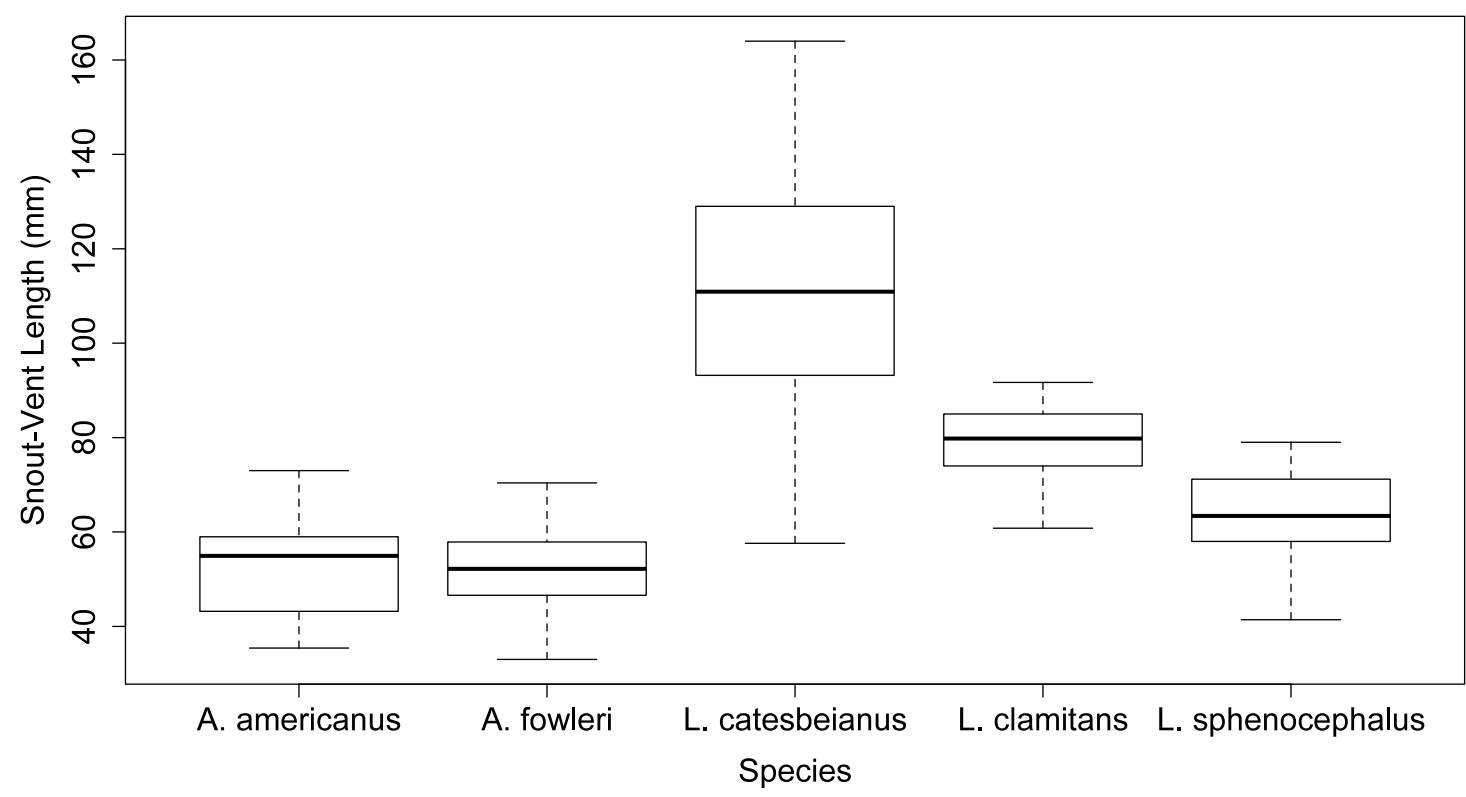

b)

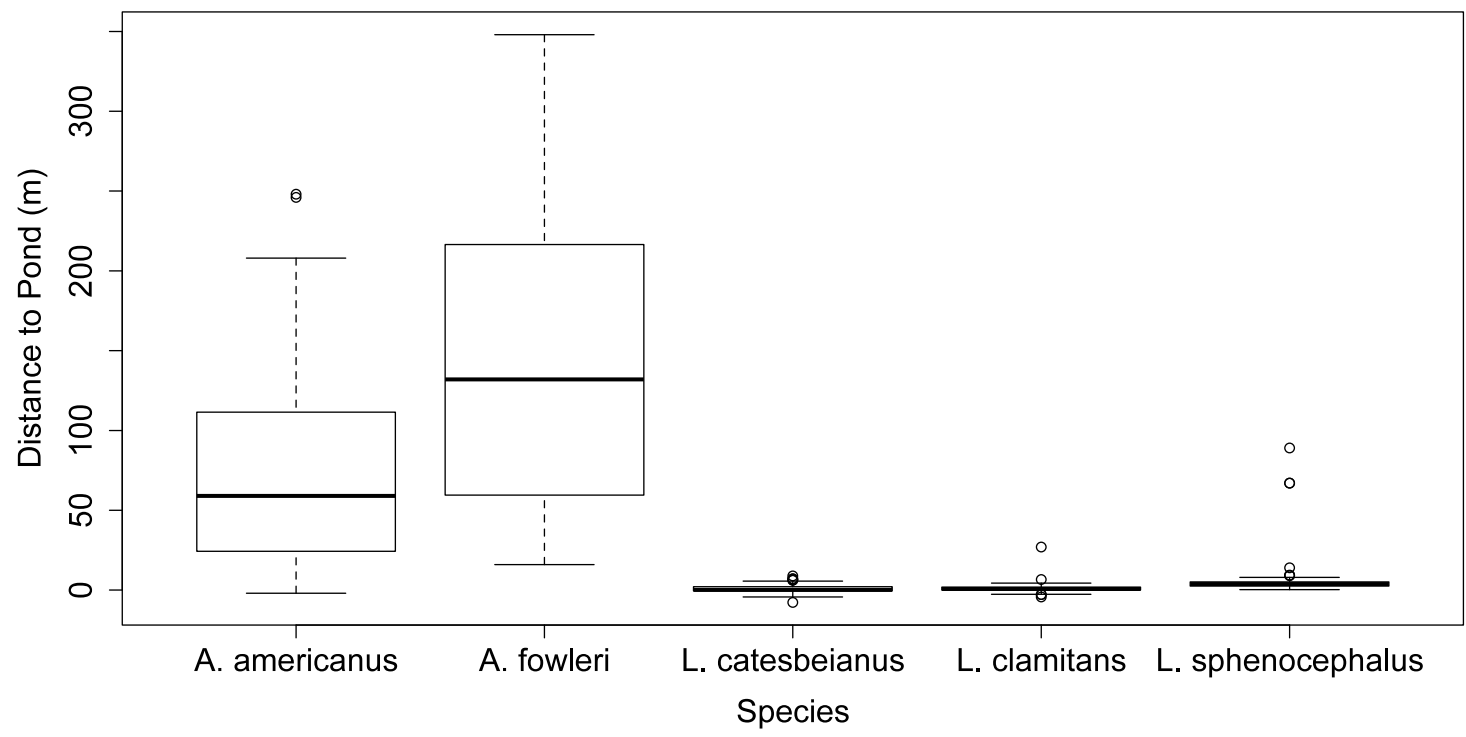


Figure 2

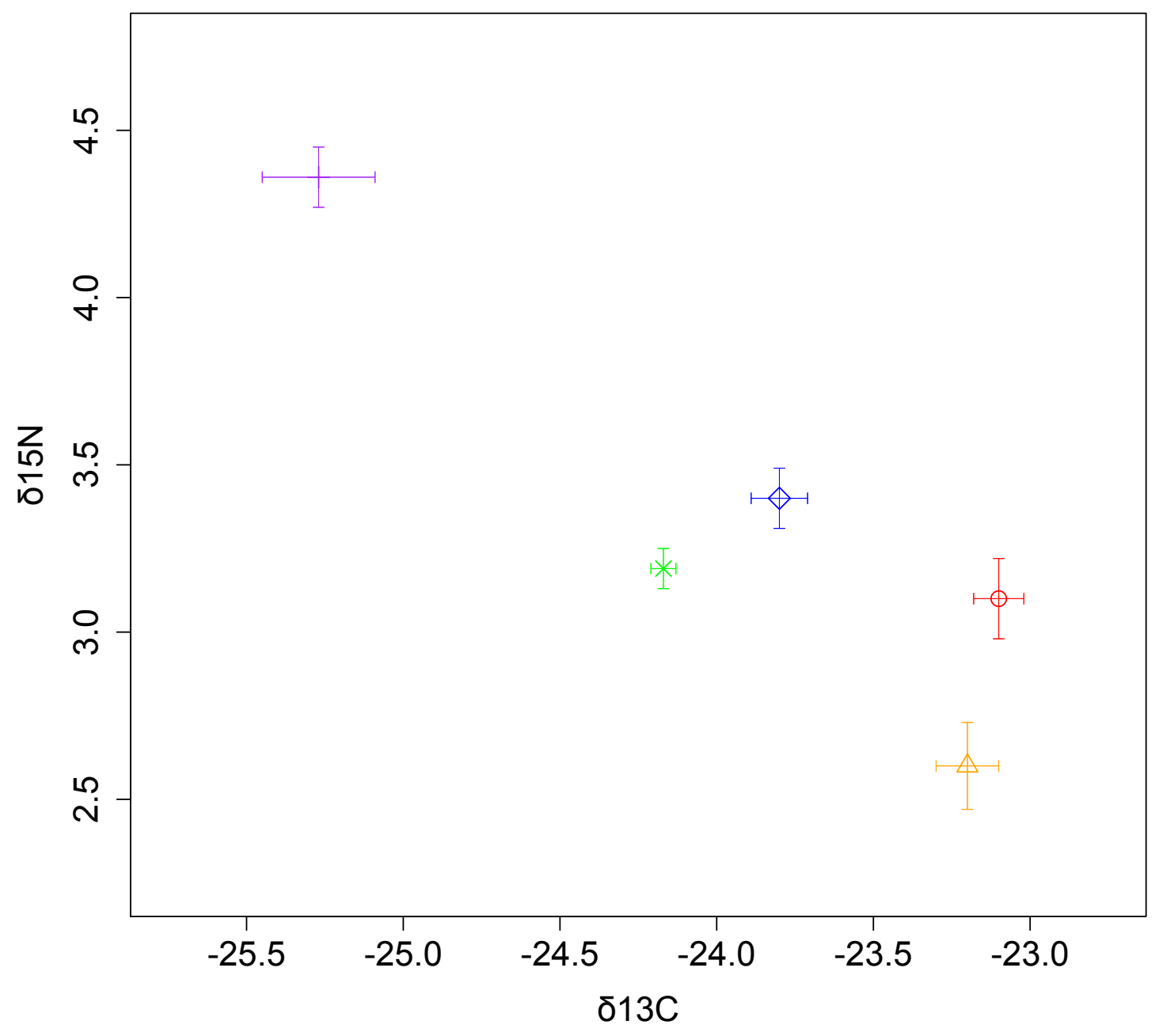


Figure 3

a)

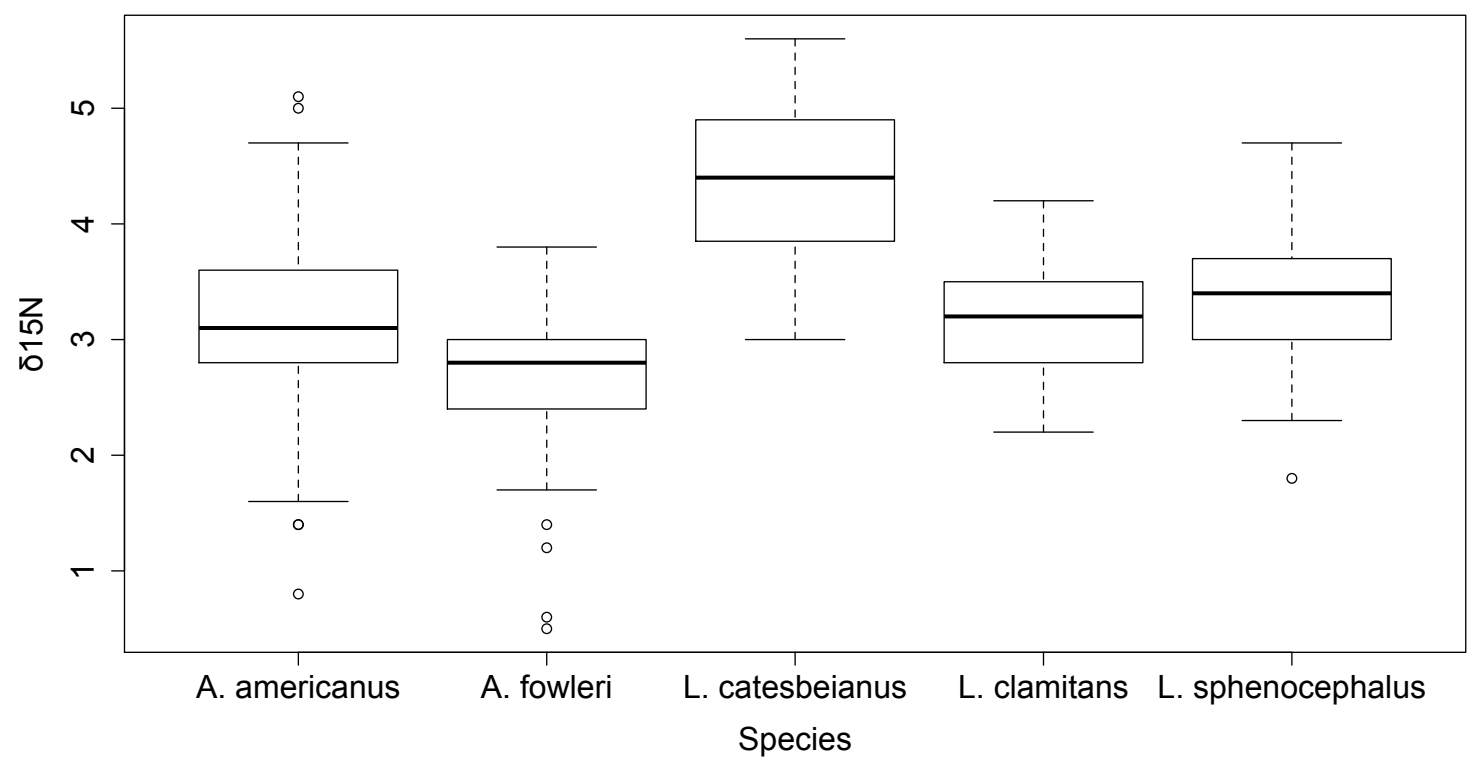

b)

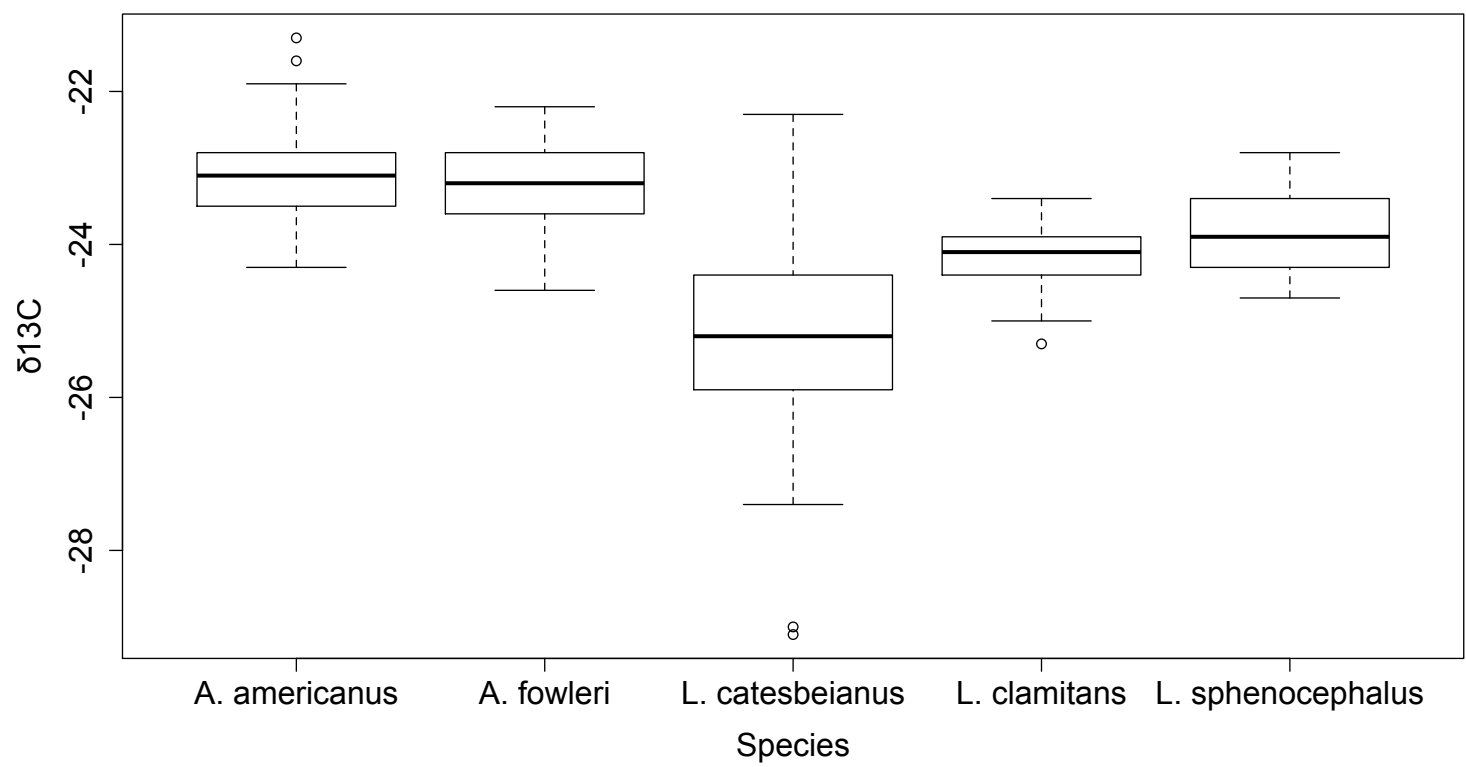


Figure 4

a)

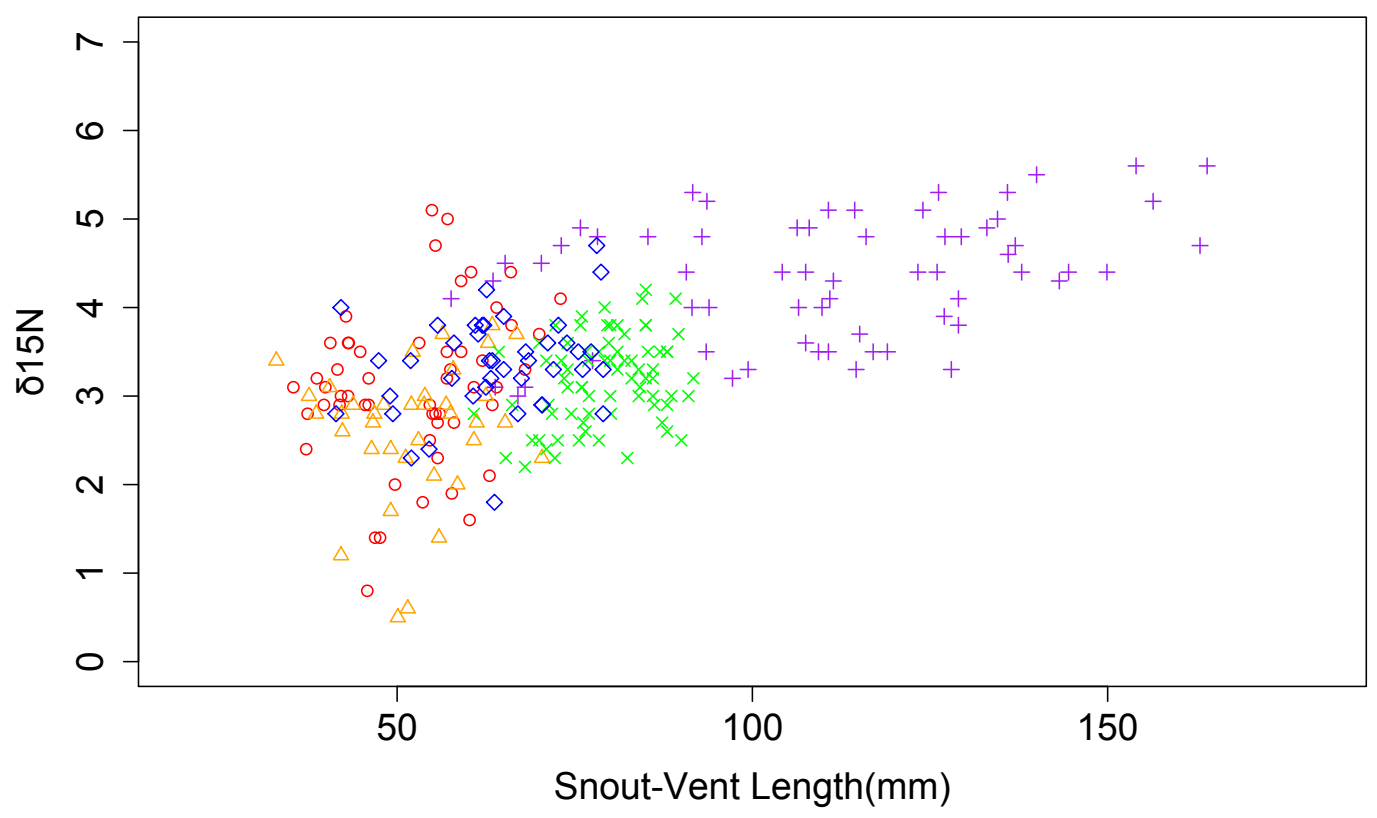

b)

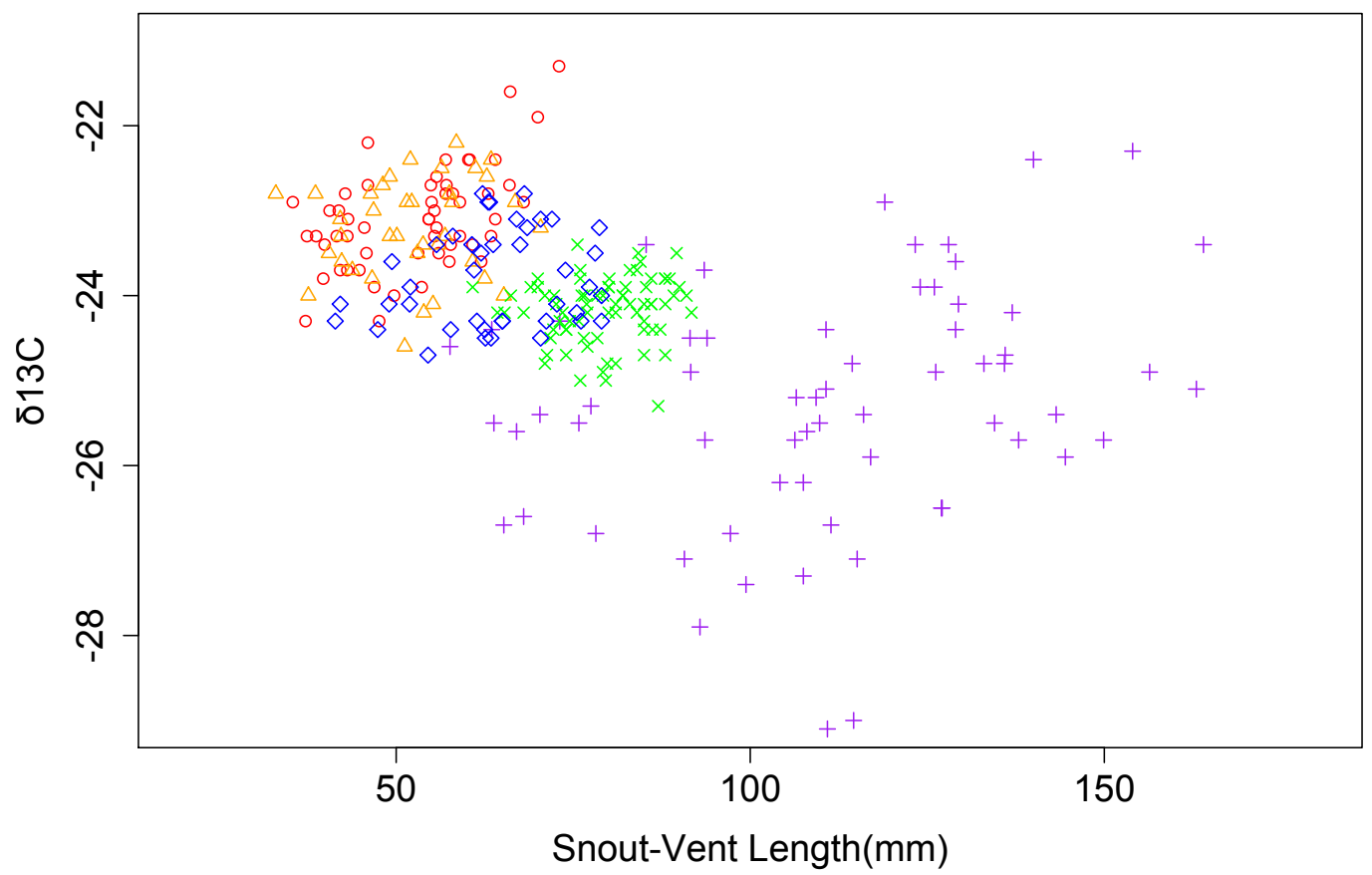


Figure 5

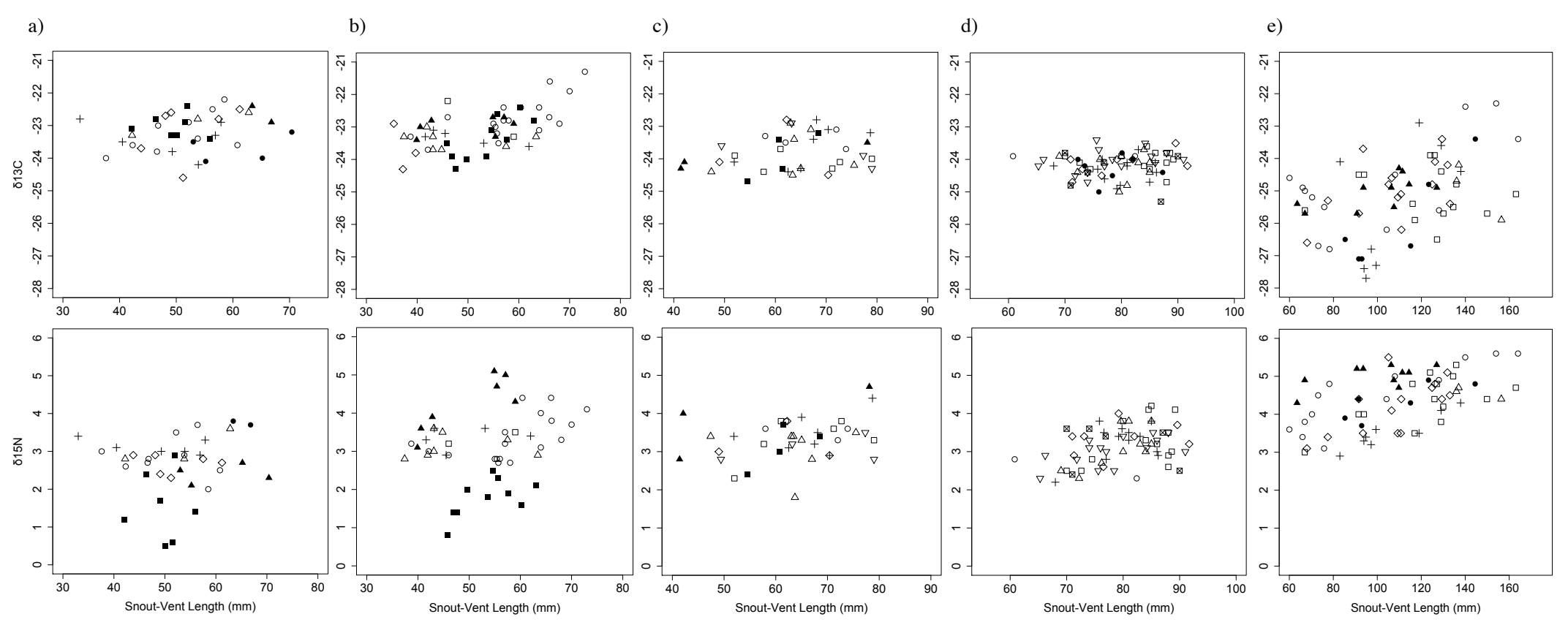


Figure 6

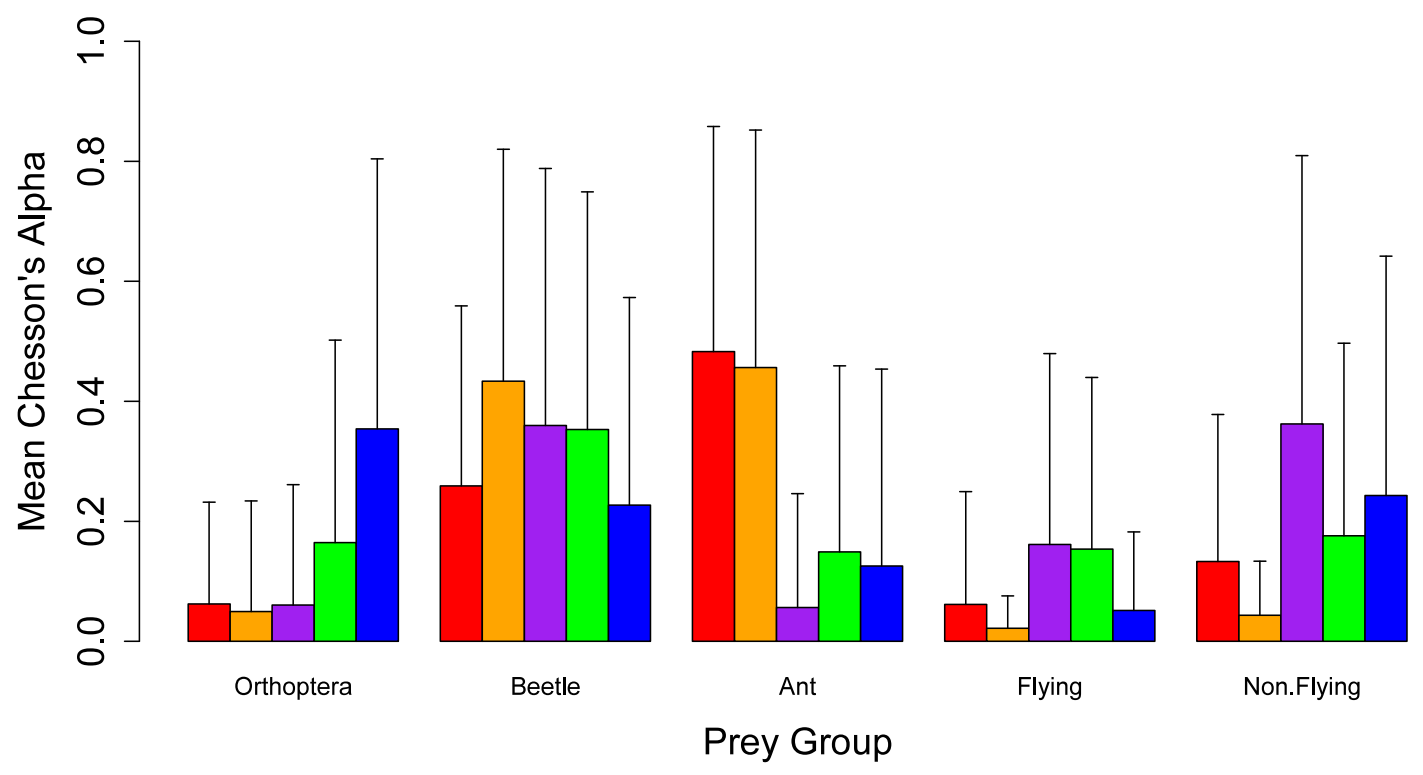




\section{CHAPTER VI \\ SUMMARY AND FUTURE DIRECTIONS}

\section{SUMMARY}

This dissertation has built a framework for considering the effects of population diversity on sympatric speciation and used that framework to test hypotheses regarding causes and consequences of between individual diet variation in a group of generalist frogs and toads. In the second chapter, I explored many types of a population diversity that can arise and how that diversity can affect speciation in sympatry. In the third chapter, I used two controlled feeding experiments to determine important stable isotope properties. In the first experiment, I determined the trophic discrimination factors for skin, whole blood, and bone collagen in adult green frogs, Lithobates clamitans. In the second experiment, I determined the isotopic incorporation rates for those same tissues. I used stable isotope analysis in the two field study chapters and this data aided that analysis. Additionally, this data will be valuable to other ecologists and conservation biologists who study frogs and toads, a globally threatened taxonomic group. In the third chapter, I measured the amount of individual specialization in five species of frogs and toads and compared how different ecological parameters affect individual specialization in each species. I found that different species had differing amounts of individual specialization and that different ecological conditions supported individual specialization 
in different species. This is a type of diversity that can last indefinitely in natural populations because individuals may switch between acting more as a specialist during certain ecological conditions and as a generalist during other conditions. In the fourth chapter, I investigated niche partitioning within the same group of frogs and toads and how individual diet differences within species can affect that niche partitioning. I found that diet variation within populations can decrease the interaction strength among competing species, aiding niche partitioning and species coexistence. This increase in the number of species within a community will result in the loss of ecological opportunity, which is required for discrete resource polymorphisms to evolve. Therefore, individual differences that support niche partitioning may help maintain themselves as indiscrete variation within populations.

\section{FUTURE WORK}

Each chapter of this dissertation has opportunities for future research. The second, fourth, and fifth chapters of the dissertation provide valuable insights into ecoevolutionary dynamics. As such, they provide us with hypotheses that we can use to better understand the relationships between ecological conditions and evolutionary dynamics. The third chapter provides vital information for ecologists and conservation biologists studying anurans and begins to fill a taxonomic gap in our knowledge of trophic discrimination factors and isotopic incorporation rates. We will separately discuss the potential future research that each chapter generated. 
There are many potential avenues of research that arise when considering the evolutionary outcomes of sympatry from the framework presented in this chapter. First, we need more documentation of individual diet variation and the mechanisms behind the variation. Increased attention needs to be paid to the genetics of these individual diet variations and researchers need to test how different genetics may lead to sexual dimorphism, protected polymorphisms, or cladogenic polymorphisms. Second, future research can attempt to gain a better understanding of the two types of cladogenic polymorphisms, those arising from developmental plasticity versus genetic variation. Researchers can work to document incidences in which morphs develop or have developed from both routes and the rates of speciation associated with each of these types of cladogenic polymorphisms. More research can test the hypothesis that the effects of fluctuating population levels and fluctuating intraspecific competition on selection pressures result in stable polymorphisms. In order to understand the final difficult steps of sympatric speciation, the relationships between genes promoting divergence of ecological trait(s) and the genes promoting reproductive isolation must be elucidated. Finally, invasive species biologists can help illuminate the role that sympatric diversification has on the colonization success of exotic species by linking the diversification processes that occurred in sympatry with the invasive ability of different subsets of the population.

While our controlled feeding experiments begin to add amphibian data to the general understanding of trophic discrimination factors and isotopic incorporation rates, more experiments need to be done on more species and on more tissues. Do these properties vary among families? For instance, do toads have different values for these properties than true frogs? And are there differences in species in tropical environments 
or for species adapted to more arid environments? Studies of this nature may be able to divide whole blood into red blood cells and blood plasma, the latter of which would have a very short halflife. Additionally, studies may include tissues such as ligaments and muscle, as they may have more intermediary turnover times between skin and bone collagen.

In the five species of frogs and toads that we studied, there was overall a low amount of individual specialization when measured with stable isotopes and a much greater amount when measured with stomach contents. Stable isotopes are likely to underestimate the degree of individual specialization because of the coarse division of prey groups. Stomach contents are likely to overestimate individual specialization in most systems because it assumes that an individual's entire diet breadth is captured in that one sampling event. Studies that use several different methods to describe diets should be used to best determine how to measure individual specialization. In particularly, those studies should combine stable isotopes with multiple other methods such as stomach content and fecal analysis on repeatedly captured individuals.

In addition to studying the best way to measure individual specializations, future work can expand on how individuals' diets respond to different ecological conditions. While our study shows that ecological parameters can increase or decrease individual specialization and that these parameters may vary among species, we do not track individuals as they become more specialists during certain ecological conditions and become generalist during other conditions. Manipulative laboratory studies on invertebrates could be performed in which individuals are subjected to periods of high and low resource diversity and their dietary decisions are compared between the two 
periods. Likewise, similar studies can be performed using different conspecific and heterospecific densities.

Finally, very few studies have investigated the effects of intrapopulation niche variation on niche partitioning. In addition to the chapter in this study, we are only aware of one study (Lasky et al. 2014) that empirically showed that variation within populations can aid niche partitioning. However, we do not know how general this relationship is. We encourage more studies on niche and resource partitioning to include individual differences in diet and/or another important niche parameter. With a better understanding of when and how these individual-level differences aid niche partitioning, we can better understand how this relationship could help maintain indefinite and indiscrete, ecologically relevant population variation. 


\section{REFERENCES}

Abbey-Lee, R. N., Gaiser, E. E., Trexler, J. C. 2013. Relative roles of dispersal dynamics and competition in determining the isotopic niche breadth of a wetland fish. Freshwater Biology 58:780-792.

Acuña, H.O., Francis, J.M. 1995. Spring and summer prey of the Juan Fernández fur seal, Arctocephalus philippii. Canadian Journal of Zoology 73:1444-1452.

Adams C. E., Huntingford F. A. 2004. Incipient speciation driven by phenotypic plasticity? Evidence from sympatric populations of Arctic charr. Biological Journal of the Linnean Society 81:611-618.

Afik D., Karasov, W. H. 1995. The trade-offs between digestion rate and efficiency in warblers and their ecological implications. Ecology 76:2247-2257.

Agashe D., Bolnick D. I. 2010. Intraspecific genetic variation and competition interact to influence niche expansion. Proceedings of the Royal Society B, Biological Sciences. 277:2915-2924.

Alexander G. D., Adams C. E. 2004. Exposure to a common environment erodes inherited between-population trophic morphology differences in Arctic charr. Journal of Fish Biology 64:253-257 
Àlvarez-Castro J. M., Alvarez G. 2005. Models of general frequency-dependent selection and mating-interaction effects and the analysis of selection patterns in Drosophilia inversion polymorphisms. Genetics 170:1167-1179.

Andersson, J., Byström, P., Persson, L., Deroos, A.M. 2005. Plastic resource polymorphism: effects of resource availability on Arctic char (Salvelinus alpinus) morphology. Biological Journal of Linnean Society 85:341-351.

Andolfatto, P. 2007. Hitchhiking effects of recurrent beneficial amino acid substitutions in the Drosophila melanogaster genome. Genome Research 17:1755-1762.

Araújo, M. S., Bolnick, D. I., Layman, C. A. 2011. The ecological causes of individual specialisation. Ecology Letters 14:948-958.

Araújo, M. S., Bolnick, D. I., Martinelli, L. A., Giaretta, A. A., dos Reis, S. F. 2009. Individual-level diet variation in four species of Brazilian frogs. Journal of Animal Ecology. 78:848-856.

Araújo, M., Guimarães, P. R. Jr., Svanbäck, R., Pinherio, A., Dos Reis, S.F., Bolnick, D.I. 2008. Network analysis reveals contrasting effects of intraspecific competition on individuals vs. population diets. Ecology 89:1981-1993.

Arnegard, M. E., McGee, M .D., Matthews, B., Marchinko, K. B., Conte, G. L., Kabir, S., Bedford, N., Bergek, S., Chan, Y. F., Jones, F. C. Kingsley, D. M., Peichel, C. L., Schulter, D. 2014. Genetics of ecological divergence during speciation. Nature 511: 307-311.

Atchley W. R., Martin J. 1971. A morphometric analysis of differential sexual 
dimorphism in larvae of Chironomus. Canadian Entomologist 103:319-327.

Awkerman, J.A., Hobson, K.A., Anderson, D.J. 2007. Isotopic $\left(\delta^{15} \mathrm{~N}\right.$ and $\left.\delta^{13} \mathrm{C}\right)$ evidence for intersexual foraging differences and temporal variation in habitat use in waved albatrosses. Canadian Journal of Zoology 85:273-279.

Barluenga, M., Meyer, A. 2010. Phylogeography, colonization, and population history of the Midas cichlid complex (Amphilophus spp.) in the Nicaraguan crater lake. BMC Evolutionary Biology 10:326.

Barluenga, M., Stölting, K. N., Salzberg, W., Muschick, M., Meyer, A. 2006. Sympatric speciation in Nicaraguan crater lake cichlid fish. Nature 43:719-723.

Barraclough, T. G., Volger, A. P. 2000. Detecting the geographical pattern of speciation from species-level phylogenies. American Naturalist 155:419-434.

Barrett, R. D. H., Schluter, D. 2007. Adaptation from standing genetic variation. Trends in Ecology and Evolution 23:38-44.

Barrionuevo, J. S., Ponssa, L. M. 2008. Decline of three species of the genus Telmatobius (Anura: Leptodactylidae) from Tucumán province, Argentina. Herpetologica 64: 47-62.

Barson, N. J., Knight, M. E., Turner, G. F. 2007. The genetic architecture of male colour differences between a sympatric Lake Malawi cichlid species pair. Journal of Evolutionary Biology 20:45-53. 
Basir, Y.J., Knoop, F.C., Dulka, J., Conlon, J.M. 2000. Multiple antimicrobial peptides and peptides related to bradykinin and neuromedin $\mathrm{N}$ isolated from skin secretions of the pickeral frog, Rana palustris. Biochimica et Biophysica Acta 1543: 95-105.

Bauchinger, U., McWilliams, S. 2009. Carbon turnover in tissues of a passerine bird: allometry, isotopic clocks, and phenotypic flexibility in organ size. Physiological and Biochemical Zoology 82: 787-797.

Bearhop, S., Adams, C.E., Waldrons, S., Fuller, R.A., Macleod, H. 2004. Determining trophic niche width: a novel approach using stable isotope analysis. Journal of Animal Ecology 73:1007-1012.

Bearhop, S., Phillips, R. A., McGill, R., Cherel, Y., Dawson, D. A., Croxall, J. P. 2006. Stable isotopes indicate sex-specific and long-term individual foraging specialization in diving seabirds. Marine Ecology Progress Series 311:157-164.

Bearhop, S., Waldon, S., Votier, S. C., Furness, R. W. 2002. Factors that influence assimilation rates, and fractionation of nitrogen and carbon isotopes in avian blood and feathers. Physiological and Biochemical Zoology 75: 451-458.

Beaulieu, M., Sockman, K. W. 2012. One meadow for two sparrows: resource partitioning in a high elevation habitat. Oecologia 170:529-540.

Bell, H. L. 1982. Sexual differences in the foraging behaviour of the frill-necked flycatcher Arses telescopthalmus in New Guinea. Australian Journal of Ecology 7, $137-147$. 
Benard, M. F., Maher, J. M. 2011. Consequences of intraspecific niche variation: phenotypic similarity increases competition among recently metamorphosed frogs. Oecologia 166:585-592.

Benkman C. W. 1996. Are the ratios of bill crossing morphs in crossbills the result of frequency-dependent selection? Evolutionary Ecology 10:119-126.

Berlocher, S. H., Feder, J. L. 2002. Sympatric speciation in phytophagous insects: moving beyond controversy? Annual Review of Entomology 47:773-815.

Berns, C. M., Adams, D. C. 2010. Bill shape and sexual shape dimorphism between two species of temperate hummingbirds: black-chinned hummingbird (Archilochus alexandri) and ruby-throated hummingbird (A. colubris). Auk 127:626-635.

Bloomfield, A.L., Elsdon, T.S., Walther, B.D., Gier, E.J., Gillanders, B.M. 2011. Temperature and diet affect carbon and nitrogen isotopes of fish muscle: can amino acid nitrogen isotopes explain effects? Journal of Experimental Marine Biology and Ecology 399:48-59.

Bolnick, D. I. 2004a. Waiting for sympatric speciation. Evolution 58:895-899.

Bolnick, D. I. 2004b. Can intraspecific competition drive disruptive selection? An experimental test in natural populations of sticklebacks. Evolution 58:608-618.

Bolnick, D. I., Amarasekare, P., Araújo, M., Bürger, R., Jiang, Y., Levine, J., Novak, M., Rudolf, V., Schreiber, S., Urban, M., Vasseur, D. 2011. Why intraspecific trait variation matters in ecology. Trends In Ecology and Evolution 26:183-192. 
Bolnick, D. I., Doebeli, M. 2003. Sexual dimorphism and adaptive speciation: two sides of the same ecological coin. Evolution 57:2433-2449.

Bolnick, D. I., Fitzpatrick, B. M. 2007. Sympatric speciation: models and empirical evidence. Annual Review of Ecology, Evolution. and Systematics 38:459-487.

Bolnick, D. I., Ingram, T., Stutz, W. E., Snowberg, L. K., Lau, O. L., Paull, J. S. 2010. Ecological release from interspecific competition leads to decoupled changes in population and individual niche width. Proceedings of the Royal Society B: Biological Sciences 277:1789-1797.

Bolnick, D. I., Lau, O. L. 2008. Predictable patterns of disruptive selection in sticklebacks in postglacial lakes. American Naturalist 172:1-11.

Bolnick, D. I., Paull, J. S. 2009. Morphological and dietary differences between individuals are weakly but positively correlated within a population of threespine stickleback. Evolutionary Ecology Research 11:1217-1233.

Bolnick, D. I., Svanbäck, R., Araújo, M. S., Persson, L. 2007. Comparative support for the niche variation hypothesis that more generalized populations also are more heterogeneous. Proceedings of the National Academy of Sciences of the United States of America 104:10075-10079.

Bolnick, D. I., Svanback, R., Fordyce, J. A., Yang, L. H., Davis, J. M., Hulsey, C. D., Forister, M. L. 2003. The ecology of individuals: incidence and implications of individual specialization. American Naturalist 161:1-28. 
Bolnick, D. I., Yang, L. H., Fordyce, J. A., Davis, J. M., Svanbäck, R. 2002. Measuring individual-level resource specialization. Ecology 83:2936-2941.

Bonk, M., Pabijan, M. 2010. Changes in a regional batrachofauna in south-central Poland over a 25 year period. Northwestern Journal of Zoology 6: 225-244.

Boone, M. D., Little, E. E., Semlitsch, R. D. 2004. Overwintered bullfrog tadpoles negatively affect salamanders and anurans in native amphibian communities. Copeia 2004:683-690.

Bosley, K. L., Witting, D.A., Chambers, R.C., Wainright, S.C. 2002. Estimating turnover rates of carbon and nitrogen in recently metamorphosed winter flounder Pseudopleuronectes americanus with stable isotopes. Marine Ecology Progress Series 236:233-240.

Brose, U., Jonsson, T., Berlow, E. L., Warren, P., Banasek-Richter, C., Bersier, L. F., Blanchard, J. L., Brey, T., Carpenter, S. R., Blandenier, M. F. C., Cushing, L., Dawah, H. A., Dell, T., Edwards, F., Harper-Smith, S., Jacob, U., Ledger, M. E., Martinez, N. D., Memmott, J., Mintenbeck, K., Pinnegar, J. K., Rall, B. C., Rayner, T. S., Reuman, D. C., Ruess, L., Ulrich, W., Williams, R. J., Woodward, G., Cohen, J. E. 2006. Consumer-resource body size relationships in natural food webs. Ecology 87:2411-2417.

Browning, N. E., Dold, C., I-Fan, J., Worthy, G. A. J. 2014. Isotope turnover rates and diet-tissue discrimination in skin of ex situ bottlenose dolphins (Tursiops truncatus). Journal of Experimental Biology 217:214-221. 
Buller, W. L. 1888. A history of the birds from New Zealand. 2 vols. $2^{\text {nd }}$ edition. London.

Bürger R. 2009. Polymorphism in the two-locus Levene model with nonepistatic directional selection. Theoretical Population Biology 76:214-228.

Burnham, K., Anderson, D. 2002. Model selection and multimodel inference: a practical information-theoretic approach. Springer, New York, NY.

Bush, F. M., Menhinick, E. F. 1962. The food of bufo woodhousei fowleri Hinckley. Herpetologica 18:110-114.

Butler, M. A., Schoener, T. W., Losos, J. B. 2000. The relationship between sexual size dimorphism and habitat use in greater Antillean Anolis lizards. Evolution 54:259272.

Calsbeek, R., Smith, T. B. 2007. Experimentally replicated disruptive selection on performance traits in a Caribbean lizard. Evolution 62:478-484.

Campagna, C., Werner, R., Karesh, W., Marin, M. R., Koontz, F., Cook, R., Koontz, C. 2001. Movements and location at sea of South American sea lions (Otaria flavescens). Journal of Zoology 2:205-220.

Campos, F.S., Brito, D., Solé, M. 2013. Threatened amphibians and their conservation status within the protected area network in northeastern Brazil. Journal of Herpetology 47:277-285.

de Cara, M. A. R., Barton, N. H., Kirkpatrick, M. 2008. A model for the evolution of assortative mating. American Naturalist 171:580-596. 
Carleton, S.A., Martínez del Rio, C. 2005. The effect of cold-induced increased metabolic rate on the rate of ${ }^{13} \mathrm{C}$ and ${ }^{15} \mathrm{~N}$ incorporation in house sparrows. Oecologia 144:226-232.

Carleton, S.A., Martínez del Rio, C. 2010. Growth and catabolism in isotopic incorporation: a new formulation and experimental data. Functional Ecology 24:805-812.

Caut, S., Angulo, E., Díaz-Paniagua, C., Gomez-Mestre, I. 2013. Plastic changes in tadpole trophic ecology revealed by stable isotope analysis. Oecologia 173: 95-105.

Caut, S., Angulo, E., Courchamp, F. 2009. Variation in discrimination factors $\left(\Delta^{15} \mathrm{~N}\right.$ and $\Delta^{13} \mathrm{C}$ ): the effect of diet isotopic values and applications for diet reconstruction. Journal of Applied Ecology 46:443-453.

Cerling, T. E., Ayliffe, L. K., Dearing, M. D., Ehleringer, J. R., Passey, B. H., Podlesak, D. W., Torregrossa, A. 2007. Determining biological tissue turnover using stable isotopes: the reaction progress variable. Oecologia 151:175-189.

Chamberlain, C. P., Waldbauer, J. R., Fox-Dobbs, K., Newsome, S. D., Koch, P. L., Smith, D. R., Church, M. E., Chamberlain, S. D., Sorenson, K. J., Risebrough, R. 2005. Pleistocene to recent dietary shifts in California condors. Proceedings of the National Academy of Scienceson the United States of America 102:16707-1671.

Chaouch, H., Hamida, O. B. A. H., Ghorbel, M., Jarboui, O. 2013. Diet composition and food habits of Diplodus puntazzo (Sparidae) from the Gulf of Gabès (Central 
Mediterranean). Journal of the Marine Biological Association of the United Kingdom 8:2257-2264.

Cherel, Y., Hobson, K. A., Hassani, S. 2005. Isotopic discrimination between food and blood and feathers of captive penguins: implications for dietary studies in the wild. Physiological and Biochemical Zoology 78:106-115.

Cherel, Y., Le Corre, M., Jaquemet, S., Ménard, F., Richard, P., \& Weimerskirch, H. 2008. Resource partitioning within a tropical seabird community: new information from stable isotopes. Marine Ecology Progress Series 366:281-291.

Chesson, J. 1983. The estimation and analysis of preference and its relationship to foraging models. Ecology 64:1297-1304.

Chesson, P. 2000. Mechanisms of maintenance of species diversity. Annual Review of Ecology and Systematics 33:343-366.

Christiansen, F. B. 1974. Sufficient conditions for protected polymorphism in a subdivided population. American Naturalist 108:157-166.

Clark, A.M. 1963. The influence of diet upon the adult life span of two species of Bracon. Annuals of the Entomological Society of America 56:616-619.

Clarke, B. C. 1969. The evidence for apostatic selection. Heredity 24:347-352.

Cloyed, C. S. 2014. Forest structure affects resource partitioning between pygmy and white-breasted nuthatches. Coevolution 2:26-30. 
Cogălnicean, D., Székely, P., Székely, D., Rosioru, D., Băncilă, R. I., Miaud, C. 2013. When males are larger than females in ectotherms: reproductive investment in the eastern spadefoot toad Pelobates syriacus. Copeia 2013:699-706.

Collins, J. P. 1979. Intrapopulation variation in the body size at metamorphosis and timing of metamorphosis in the bullfrog, Rana catesbeiana. Ecology 60:738-749.

Collins J. P., Cheek J. E. 1983. Effect of food and density on development of typical and cannibalistic salamander larvae in Ambystoma tigrinum nebulosum. American Zoology 23:77-84.

Conde-Padín, P., Carvajal-Rodríguez, A., Carballo, M., Caballero, A., Rolán-Alvarez, E. 2007. Genetic variation for shell traits in a direct-developing marine snail involved in a putative sympatric ecological speciation. Evolutionary Ecology 21:635-650.

Connan, M., McQuaid, C. D., Bonnevie, B. T., Smale, M. J., Cherel, Y. 2014. Combined stomach content, lipid and stable isotope analyses reveal spatial and trophic partitioning among three sympatric albatrosses from the Southern Ocean. Marine Ecology Progress Series 497: 259-272.

Connallon, T., Knowles, L. L. 2007. Recombination rate and protein evolution in yeast. BMC Evolutionary Biology 7:235-242.

Cook, L. M. 2007. Heterosis in Cepaea. Biological Journal of the Linnaean Society 90:49-53. 
Correa, S. B., Winemiller, K. O. 2014. Niche partitioning among frugivorous fishes in response to fluctuating resources in the Amazonian floodplain forest. Ecology 95:210-224.

Costa, G. C., Mesquita, D. O., Colli, G. R., Vitt, L. J. 2008. Niche expansion and the niche variation hypothesis: does the degree of individual variation increase in depauperate assemblages? American Naturalist 172:868-877.

Coyne, J. A., Meyers, W., Crittenden, A. P., Sniegowski, P. 1993. The fertility effects of pericentric inversions in Drosophila melanogaster. Genetics 134:487-496.

Coyne J. A., Price T. D. 2000. Little evidence for sympatric speciation in island birds. Evolution 54:2166-2171.

Coyne, J. A., Orr, H. A. 2004. Speciation. Sinauer, Sunderland, MA.

Crow, K. D., Munehara, H., Bernard, G. 2010. Sympatric speciation in a genus of marine reef fishes. Molecular Ecology 19:2089-2105.

Dalerum, F., Angerbjörn, A. 2005. Resolving temporal variation in vertebrate diets using naturally occurring stable isotopes. Oecologia 144:647-658.

Dambroski, H. R., Feder, J. L. 2007. Host plant and latitude-related diapause variation in Rhagoletis pomonella: a test for multifaceted life history adaptation on different stages of diapause development. Journal of Evolutionary Biology 20:2101-2112. 
Darimont, C. T., Paquet, P. C., Reimchen, T. E. 2007. Stable isotopic niche predicts fitness of prey in a wolf-deer system. Biological Journal of the Linnaean Society $9: 125-137$.

Darimont, C. T., Paquet, P. C., Reimchen, T. E. 2009. Landscape heterogeneity and marine subsidy generate extensive intrapopulation niche diversity in a large terrestrial vertebrate. Journal of Animal Ecology 78:126-133.

Darwin, C. 1859. On the origins of species by the means of natural selection, or the preservation of favored races in the struggle for life. John Murray Press, London, U.K.

Darwin, C. 1871. The descent of man and selection in relation to sex. John Murray Press, London, U.K.

David, P. 1998. Heterozygosity - fitness correlations: new perspectives on old problems. Heredity 80:531-537.

DeNiro, M. J., Epstein, S. 1978. Influence of diet on the distribution of carbon isotopes in animals. Geochimica et Cosmochimica Acta 42: 495-506.

DeWitt, T. J., Sih, A., Wilson, D. S. 1998. Costs and limits of phenotypic plasticity. Trends in Ecology and Evolution 13:77-81.

Débarre, F. 2012. Refining the conditions for sympatric ecological speciation. Journal of Evolutionary Biology 25:2651-2660. 
Dieckmann, U., Doebeli, M. 1999. On the origin of species by sympatric speciation. Nature 400:354-357.

Diegisser, T., Johannesen, J., Seitz, A. 2007. Performance of host-races of the fruit fly, Tephritis conura on a derived host plant, the cabbage thistle Cirsium oleraceum: implications for the original host shift. Journal of Insect Science 8, 66.

Ding, A., Goudet, J. 2005. Heterozygote advantage and the maintenance of polymorphism for multilocus traits. Theoretical Population Biology 68:157-166.

Dobzhansky, T. 1937. Genetics and the Origin of Species. Columbia University Press, New York City, NY.

Doebeli, M. 1996. A quantitative genetic competition model for sympatric speciation. Journal of Evolutionary Biology 9:893-909.

Doebeli, M., Dieckmann, U. 2000. Evolutionary branching and sympatric speciation caused by different types of ecological interactions. American Naturalist 156:S77S101.

Doebeli, M., Blok, H. J., Leimar, O., Dieckmann, U. 2007. Multimodal pattern formation in phenotype distributions of sexual populations. Proceedings of the Royal Society B: Biological Sciences 274:347-357.

Dohi, Y., Tabata, S., Yamaguchi, M., Ohgushi, H., Yonemasu, K. 2004. Characterization of the cDNA encoding bullfrog, Rana catesbeiana, osteocalcin and two forms of the protein isolated from bone. Biochimie 86: 471-480. 
Drès, M., Mallet, J. 2002. Host races in plant-feeding insects and their importance in sympatric speciation. Philosophical Transactions of the Royal Society B: Biological Sciences 357:471-492.

du Toit, J. 2005. Sex differences in the foraging ecology of large mammalian herbivores. Pages 35-52 in Sexual Segregation in Vertebrates: Ecology of the Two Sexes edited by K. E. Ruckstuhl, P. Neuhaus. Cambridge (United Kingdom): Cambridge University Press.

Ellerby, D. J., Gerry, S. P. 2011. Sympatric divergence and performance trade-offs of bluegill ecomorphs. Evolutionary Biology 38:422-433.

Elmer, K. R., Meyer, A. 2011. Adaptations in the age of ecological genomics: insights from parallelism and convergence. Trends in Ecology and Evolution 26:298-306.

Emelianov, I., Drès, M., Baltensweiler, W., Mallet, J. 2001. Host-induced assortative mating in host races of the larch budmoth. Evolution 55:2002-2010.

Endler, J. A. 1992. Signals, signal conditions, and the direction of evolution. American Naturalist 139:S125-S153.

Estes, J. A., Riedman, M. L., Staedler, M. M., Tinker, M. T., Lyon, B. E. 2003. Individual variation in prey selection by sea otters: patterns, causes, and implications. Journal of Animal Ecology 72:144-155.

Evangelista, C., Boiche, A., Lecerf, A., Cucherousset, J. 2014. Ecological opportunities and intraspecific competition alter trophic niche specialization in an opportunistic stream predator. Journal of Animal Ecology 83:1025-1034. 
Ewart, J. P. 1974. The neural basis of visually guided behavior. Scientific American 230:34-42.

Ewart, J. P. 2004. Motion perception shapes the visual world of amphibians. Pages 117160 in Complex worlds from simpler nervous systems. Cambridge, (MA): MIT Press.

Fan, S., Elmer, K. R., Meyer, A. 2012. Genomics of adaptation and speciation in cichlid fishes: recent advances and analyses in African and Neotropical lineages. Proceedings of the Royal Society B: Biological Sciences 367:385-394.

Feder J. L., Chilcote C. A., Bush G. L. 1990. The geographic pattern of genetic differentiation between host associated populations of Rhagoletis pomonella (Diptera: Tephritidae) in the eastern United States and Canada. Evolution 80:277283.

Feder, J. A., Berlocher, S. H., Roethele, J. B., Dambroski, H., Smith, J. J., Perry, W. L., Gavrilovic, V., Filchak, K. E., Rull, J., Aluja, M. 2003. Allopatric genetic origins for sympatric host-plant shifts and race formation in Rhagoletis. Proceedings of the National Academy of Sciences of the United States of America 100:10314-10319.

Ficetola, G. F., Bernardi, F. D. 2006. Trade-off between larval development rate and post-metamorphic traits in the frog Rana latastei. Evolution and Ecology 20:143158. 
Fisk, A. T., Sash, K., Maerz, J., Palmer, W., Carroll, J. P., MacNeil, M. A. 2009. Metabolic turnover rates of carbon and nitrogen stable isotopes in captive juvenile snakes. Rapid Communication Mass Spectrometry 23:319-326.

Fitzpatrick B. M., Fordyce J. A., Gavrilets S. 2008. What, if anything, is sympatric speciation. Journal of Evolutionary Biology 21:1452-1459.

Fitzpatrick B. M., Turelli M. 2006. The geography of mammalian speciation: mixed signals from phylogenies and range maps. Evolution 60:601-615.

Florin, S. T., Felicetti, L. A., Robbins, C. T. 2011. The biological basis for understanding and predicting dietary-induced variation in nitrogen and sulphur isotope ratio discrimination. Functional Ecology 25: 519-526.

Foote, A. D., Newton, J., Piertney, S. B., Willerslev, E., Gilbert, M. T. P. 2009. Ecological, morphological, and genetic divergence of sympatric north Atlantic killer whale populations. Molecular Ecology 18:5207-5217.

Forero, M.G., González-Solís, Hobson, K.A., Donázar, J.A., Bertellotti, M., Blano, G., Bortolotti, G.R. 2005. Stable isotopes reveal trophic segreation by sex and age in the southern giant petrel in two different food webs. Marine Ecology Progress Series 296:107-113.

Forero, M.G., Hobson, K.A., Bortolotti, G.R., Donázar, J.A., Bertellotti, M., \& Blano, G. 2002. Food resource utilization by the Magellanic penguin evaluated through stable-isotope analysis: segregation by sex and age and influenece on offspring quality. Marine Ecology Progress Series 234:289-299. 
Forester, D. C., Snodgrass, J. W., Marsalek, K., Lanham, Z. 2006. Post-breeding dispersal and summer home range of female American toads (Bufos americanus). Northeastern Naturalist 13:59-72.

Frédérich, B., Lehanse, O., Vandewalle, P., Lepoint, G. 2010. Trophic niche width, shift, and specialization of Dascyllus aruanus in Toliara Lagoon, Madagascar. Copeia 2:218-226.

Frere, E., Quintana, F., Gandini, P., Wilson, R. P. 2008. Foraging behaviour and habitat partitioning of two sympatric cormorants in Patagonia, Argentina. Ibis 150:558564.

Fry, B. 2006. Stable Isotope Ecology. Springer, New York, NY.

Futuyma, D. J., Mayer, G.C. 1980. Non-allopatric speciation in animals. Systematic Zoology 29:254-271.

Gause, G. F. 1932. Experimental studies on the struggle for existence. Journal of Experimental Biology 9:389-402.

Gavrilets, S. 2005. “Adaptive speciation"-It is not that easy: a reply to Doebeli et al. Evolution 59:696-699.

Gavrilets, S., Vose, A. 2005. Dynamic patterns of adaptive radiation. Proceedings of the National Academy of Sciences of the United States of America 102:18040-18045

Geiger, M. F., McCrary, J. K., Schliewen, U. 2010. Not a simple case-a first comprehensive phylogenetic hypothesis for the Midas cichlid complex in Nicaragua 
(Teleostei: Cichlidae: Amphilophus). Molecular Phylogenetics and Evolution $56: 1011-1024$.

Ghalambor, C. K., McKay, J. K., Carroll, S. P., Reznick, D. N. 2007. Adaptive versus non-adaptive phenotypic plasticity and the potential for contemporary adaptation in new environments. Functional Ecology 21:394-407.

Graeter, G. J., Rothermel, B. B., Gibbons, J. W. 2008. Habitat selection and movement of pond-breeding amphibians in experimentally fragmented pine forests. Journal of Wildlife Management 72:473-482.

Grant, P. R., Grant, B. R. 2008. How and why species multiply. Princeton University Press, Princeton, NJ.

Grant, M., Robison, A., Fincke, O. M. 2014. Use of stable isotopes to assess the intraspecific foraging niche of males and female colour morphs of the damselfly Enallagma hageni. Ecological Entomology 39:109-117.

González-Gómez, P. L., Estades, C. F. 2009. Is natural selection promoting sexual dimorphism in the green-backed firecrown hummingbird (Sephanoides sephaniodes)? Journal of Ornithology 150:351-356.

Gotanda, K. M., Turgeon, K., Kramer, D. L. 2009. Body size and reserve protection affect flight initiation distance in parrotfishes. Behavioral Ecology and Sociobiology 63:1563-1572.

Gould-Somero, M. 1975. Echiura. Pages 277-312 in Reproduction in Marine Invertebrates Vol. 3 edited by A. Giese, J. S. Pearse. New York (U.S.A.) Academic Press. 
Hairston, N. G., Dillon, T. A. 1990. Fluctuating selection and response in a population of freshwater copepods. Evolution 44:1796-1805.

Hamilton, Jr., W. J. 1948. The food and feeding behavior of the green frog, Rana clamitans Latreille, in New York State. Copeia 1948:203-207.

Hamilton, Jr., W. J. 1954. The economic status of the toad. Herpetologica 10:37-40.

Hampton, P. M. 2011. Feeding performance in the western ribbon snake (Thamnophis proximus): ontogeny and the effects of prey type and size. Canadian Journal of Zoology 89:945-950.

Hardin, G. 1960. The competitive exclusion principle. Science 131:1292-1297.

Hare, P. G., Fogel, M. L., Stafford Jr., T.W., Mitchell, A.D., Hoering, T.C. 1991. The isotopic composition of carbon and nitrogen in individual amino acids isolated from modern and fossil proetins. Journal of Archaeological Science 18:277-292.

Harper, E. B., Semlitsch, R. D. 2007. Density dependence in the terrestrial life history stage of two anurans. Oecologia 153:879-889.

Hasumi, M. 2010. Age, body size, and sexual dimorphism in size and shape in Salamandrella keyserlingii (Caudate: Hynobiidae). Evolutionary Biology 37:38-48.

Hauffe, H. C., Searle, J. B. 1993. Extreme karyotypic variation in a Mus musculus domesticus hybrid zone: the tobacco mouse story revisited. Evolution 47:13741395.

Hawthrone, D. J., Via, S. 2001. Genetic linkage of ecological specialization and reproductive isolation in pea aphids. Nature 412:904-907. 
Heady, W. N., Moore, J. W. 2013. Tissue turnover and stable isotope clocks to quantify resource shifts in anadromous rainbow trout. Oecologia 172: 21-34.

Hecnar, S. J., M'Closky, R. T. 1997. Changes in the composition of a ranid frog community following bullfrog extinction. American Midland Naturalist 137:145150.

Hedrick, P. W., Ritland, K. 2011. Population genetics of the white-phased "spirit" black bear of British Columbia. Evolution 66:305-313.

Hendry, A. P. 2001. Adaptive divergence and the evolution of reproductive isolation in the wild: an empirical demonstration using introduced sockeye salmon. Genetica 112-113: 515-534.

Hendry, A. P. 2009. Ecological speciation! Or lack thereof? Canadian Journal of Fisheries and Aquatic Science 66:1383-1398.

Hendry, A. P., Bolnick D. I., Berners D., Peichel C. L. 2009a. Along the speciation continuum in sticklebacks. Journal of Fish Biology 75:2000-2036.

Hendry, A. P., Grant, P. R., Grant, B. R., Ford, H. A., Brewer, M. J., Podos, J. 2006. Possible human impacts on adaptive radiation: beak size bimodality in Darwin's finches. Proceedings of the Royal Society B: Biological Sciences 273:1887-1894.

Hendry, A. P., Huber, S. K., de León, L. F., Herrel, A., Podos, J. 2009b. Disruptive selection in a model population of Darwin's finches. Proceedings of the Royal Society B: Biological Sciences 276:753-759. 
Hendry, A. P., Taylor, E. B., McPhail, J. D. 2002. Adaptive divergence and the balance between selection and gene flow: lake and stream stickleback in the misty system. Evolution 56:1199-1216.

Hendry, A. P., Wenburg, J. K., Bentzen, P., Volk, E., Quinn, T. P. 2000. Rapid evolution of reproductive isolation in the wild: evidence from introduced salmon. Science 290:516-518.

Herrera, L. G., Korine, C., Fleming, T. H., Arad, Z. 2008. Dietary implications of intrapopulation variation in nitrogen isotope composition of an old world fruit bat. Journal of Mammalogy 89:1184-1190.

Heupel, M. R., Knip, D. M., Simpfendorfer, C. A., Dulvy, N. K. 2014. Sizing up the ecological role of sharks as predators. Marine Ecology Progress Series 495:291298.

Hey J., Kliman R. M. 2002. Interactions between natural selection, recombination and gene density in the genes of Drosophila. Genetics 160:595-608.

Hirai, T., Matsui, M. 2002. Feeding relationships between Hyla japonica and Rana nigromaculata in rice fields of Japan. Journal of Herpetology 36:662-667.

Hobson, K. A., Clark, R. G. 1992a. Assessing avian diets using stable isotopes II: factors influencing diet-tissue fractionation. The Condor 94: 189-197.

Hobson, K. A., Clark, R. G. 1992b. Assessing avian diets using stable isotopes I: turnover of ${ }^{13} \mathrm{C}$ in tissues. The Condor 94: 181-188. 
Hobson, K. A., Yohannes, E. 2007. Establishing elemental turnover in exercising birds using a wind tunnel: implications for stable isotope tracking of migrants. Canadian Journal of Zoology 85: 703-708.

Hoekstra, H. E., Hirschmann, R. J., Bundey, R. J., Insel, P., Crossland, J. P. 2006. A single amino acid mutation contributes to adaptive color pattern in beach mice. Science 313:101-104.

Hoen, D. K., Kim, S. L., Hussey, N. E., Wallsgrove, N. J., Drazen, J. C., Popp, B. N. 2014. Amino acid ${ }^{15} \mathrm{~N}$ trophic enrichment factors of four large carnivorous fishes. Journal Experimental Marine Biology and Ecology 453:76-83.

Höglund, N. H. 1964. Über die Ernährung des Habichts (Accipter gentilis Lin.) in Schweden. Viltrevy 2:271-328.

Holyoak, D. T. 1970. Sex differences in feeding behaviour and size in the carrion crow. Ibis 112:397-400.

Homan, R. N., Atwood, M. A., Dunkle, A. J., Karr, S. B. 2010. Movement orientation by adult and juvenile wood frogs (Rana sylvatica) and American toads (Bufo americanus) over multiple years. Herpetological Conservation and Biology 5:6472.

Hori, M. 1993. Frequency-dependent natural selection in the handedness of scale-eating cichlid fish. Science 260:216-219.

Howland, M. R., Corr, L. T., Young, S. M. M., Jones, V., Jim, S., van der Merwe, N. J., Mitchell, A. D., Evershed, R. P. 2003. Expression of the dietary isotope signal in 
the compound-specific $\delta^{13} \mathrm{C}$ values of pig bone lipids and amino acids. International Journal of Osteoarchaeology 13:54-65.

Huber, S. K., de León, L. F., Hendry, A. P., Bermingham, E., Podos, J. 2007. Reproductive isolation of sympatric morphs in a population of Darwin's finches. Proceedings of the Royal Society, London, Biological Sciences. 274, 1709-1714.

Hughes A. L., Hughes M.K. 1986. Paternal investment and sexual size dimorphism in North American passerines. Oikos 46:171-175.

Huckembeck, S., Loebmann, D., Albertoni, E. F., Hefler, S. M., Oliveira, C. L. M., Garcia, A. M. 2014. Feeding ecology and basal food sources that sustain the paradoxal frog Pseudis minuta: a multiple approach combining stomach content, prey availability, and stable isotopes. Hydrobiologia 740:253-264.

Hussey, N. E., Brush, J., McMarth, I. D., Fisk, A.T. 2010. $\delta^{15} \mathrm{~N}$ and $\delta^{13} \mathrm{C}$ diet-tissue discrimination factors for large sharks under semi-controlled conditions. Comparative Biochemistry and Physiology A 155:445-453.

Hutchinson, G. E. 1957. Concluding remarks. Cold Spring Harbor Symposia on Quantitative Biology 22:415-427.

Hutchinson, G. E. 1959. Homage to Santa Rosalia or why are there so many kinds of animals? American Naturalist 93:145-159.

Hutchinson, G. E. 1961. The paradox of the plankton. American Naturalist 95:137-145. 
Innan, H., Kim, Y. 2004. Pattern of polymorphism after strong artificial selection in a domestication event. Proceedings of the National Academy of Sciences of the United States of America 101:10667-10672.

Jansen, O. E., Michel, L., Lepoint, G., Das, K., Couperus, A. S., Reijnders, J. H. 2013. Diet of harbor porpoises along the Dutch coast: a combined stable isotope and stomach contents approach. Marine Mammal Science 29:E295-E311.

Jefferson, D. M., Russell, R. W. 2008. Onogenetic and fertilizer effects on stable isotopes in the green frog (Rana clamitans). Applied Herpetology 5:189-196.

Jiang, Y., Bolnick, D. I., Kirkpatrick, M. 2013. Assortative mating in animals. American Naturalist 181:125-138.

Jin, L, Li, Q., Song, S., Feng, K., Zhang, D., Wang, Q., Chen, Y. 2009. Characterization of antimicrobial peptides isolated from the skin of the Chinese frog, Rana dybowskii. Comparative Biochemistry and Physiology B 154: 174-178.

Johnson, J. B., Omland, K. S. 2004. Model selection in ecology and evolution. Trends in Ecology and Evolution 19:101-108.

Jones, F. C., Grabherr, M. G., Chan, Y. F. 2012. The genomic basis of adaptive evolution in threespine stickleback. Nature 484:55-61.

Jones, A. W., Post, D. M. 2013. Consumer interaction strength may limit the diversifying effect of intraspecific competition: a test in alewife (Alosa pseudoharengus). American Naturalist 181:815-826. 
Jourdan-Pineau, H., David, P., Crochet, P. 2012. Phenotypic plasticity allows the Mediterranean parsley frog Pelodytes punctatus to exploit two temporal niches under continuous gene flow. Molecular Ecology 21:876-886.

Kamilar, J. M., Pokempner, A. A. 2008. Does body mass dimorphism increase malefemale dietary niche separation? A comparative study of primates. Behavior 145:1211-1234.

Karlin, S., Campbell, R.B. 1981. The existence of a protected polymorphism under conditions of soft as opposed to hard selection. American Naturalist 117:262-275.

Kautt, A. F., Elmer, K. R., Meyer, A. 2012. Genomic signatures of divergent selection and speciation patterns in a 'natural experiment', the young parallel radiations of Nicaraguan crater lake cichlid fishes. Molecular Ecology 21:4770-4786.

Kilman, L. 1965. Differences in feeding behavior of male and female hairy woodpeckers. Wilson Bulletin 77:134-145.

Kilpatrick, A. M., Briggs, C. J., Daszak, P. 2010. The ecology and impact of chytridiomycosis: an emerging disease in amphibians. Trends in Ecology and Evolution 25:109-118.

Kim, S. L., Casper, D. R., Galván-Magaña, F., Ochoa-Díaz, R., Hernández-Aguilar, S. B., Koch, P. L. 2012. Carbon and nitrogen discrimination factors for elasmobranch soft tissues based on a long-term controlled feeding study. Environmental Biology of Fish 95:37-52. 
King, J. E. 1983. Seals of the World. British Journal of Natural History and Oxford University Press, London, U.K.

Kingsolver, J. G., Hoekstra, H. E., Hoekstra, J. M., Berrigan, D., Vignieri, S. N., Hill, C. E., Hoang, A., Gibert, P., Beerli, P. 2001. The strength of phenotypic selection in natural populations. American Naturalist 157:245-261.

Kirkpatrick, M., Ravingé, V. 2002. Speciation by natural and sexual selection: models and experiments. American Naturalist 159(S3):S22-S35.

Knickle, D. C., Rose, G. A. 2014. Dietary niche partitioning in sympatric gadid species in coastal Newfoundland: evidence from stomachs and C-N isotopes. Environmental Biology of Fish 97:343-355.

Knudsen, R., Klemetsen, A., Amundsen, P. A., Hermansen, B. 2006. Incipient speciation through niche expansion: an example from the Arctic charr in a subarctic lake. Proceedings of the Royal Academy London B: Biological Sciences 273:2291-2298.

Kolts, J. M., Lovvorn, J. R., North, C. A., Grebmeier, J. M., Cooper, L. W. 2013. Relative value of stomach contents, stable isotopes, and fatty acids as diet indicators for a dominant invertebrate predator (Chionoecetes opilio) in the northern Bering Sea. Journal of Experimental Marine Biology and Ecology 449:274-283.

Kopp, M., Hermisson, J. 2008. Competitive speciation and costs of choosiness. Journal of Evolutinoary Biology 21:1005-1023. 
Kornicker, L. S. 1985. Sexual dimorphism, ontogeny and functional morphology of Rutiderma hartmanni Poulsen, 1965 (Crustacea: Ostracoda). Smithsonian Contributions to Zoology 408:1-28.

Kurle, C. M. 2009. Interpreting temporal variation in omnivore foraging ecology via stable isotope modeling. Functional Ecology 23:733-744.

Lande R. 1982. Rapid origin of sexual isolation and character divergence in a cline. Evolution 36:213-223.

Lasky, J. R., Yang, J., Zhang, G., Cao, M., Tang, Y., Keitt, T. H. 2014. The role of functional traits and individual variation in the co-occurrence of Ficus species. Ecology 95:978-990.

Layman, C. A., Araújo, M. S., Boucek, R., Hammerschlag-Peyer, C. M., Harrison, E., Jud, Z. R., Matich, P., Rosenblatt, A. E., Vaudo, J. J., Yeager, L. A., Post, D. M., Bearhop, S. 2012. Applying stable isotopes to examine food-web structure: an overview of analytical tools. Biological Reviews of the Cambridge Philosophic Society 87:545-562.

Le Boeuf, B. J., Crocker, D. E., Costa, S. B., Blackwell, P. M., Houser, D. S. 2000. Foraging ecology of northern elephant seals. Ecological Monographs 70:353-382.

Lesage, V., Hammill, M.O., Kovacs, K.M. 2002. Diet-tissue fractionation of stable carbon and nitrogen isotopes in phocid seals. Marine Mammal Science 18:182-193. 
Lesage, V., Hammill, M. O., Kovacs, K. M. 2001. Marine mammals and the community structure of the Estuary and Gulf of St. Lawrence, Canada: evidence from stable isotope analysis. Marine Ecology Progress Series 210:203-221.

Levene, H. 1953. Genetic equilibrium when more than one ecological niche is available. American Naturalist 87:331-333.

Lewontin, R. C. 1958. A general method for investigating the equilibrium of gene frequency in a population. Genetics 43:419-434.

Li, Y., Z. Ke, Y. Wang, and T. M. Blackburn. 2011. Frog community responses to recent American bullfrog invasions. Current Zoology 57:83-92.

Lichstein, J. W., Dushoff, J., Levin, S. A., Pacala, S. W. 2007. Intraspecific variation and species coexistence. American Naturalist 170:807-818.

Liordos, V., Goutner, V. 2009. Sexual differences in the diet of great cormorants Phalacrocorax carbo sinensis wintering in Greece. European Journal of Wildlife Research 55:301-308.

Livezey, B. C., Storer, R. W. Morphometric comparison of skeletons of the western grebe complex Aechmophorus of the United States and Canada. Condor 94:668-679.

Logan, J., Haas, H., Deegan, L., Gaines, E. 2006. Turnover rates of nitrogen stable isotopes in the salt marsh mummichog, Fundulus heteroclitus, following a laboratory diet switch. Oecologia 147:391-395. 
Logan, J. M., Lutcavage, M. E. 2010. Stable isotope dynamics in elasmobranch fishes. Hydrobiologica 644:231-244.

Lorrain, A., Graham, B. S., Ménard, F., Popp, B. N., Bouillon, S., van Breugel, P., Cherel, Y. 2009. Nitrogen and carbon isotope values of individual amino acids: a tool to study foraging ecology of penguins in the Southern Ocean. Marine Ecology Progress Series 391:293-306.

Losos J. B. 2010. Adaptive radiation, ecological opportunity, and evolutionary determinism. American Naturalist 175:623-639.

Maan, M. E., Seehausen, O. 2011. Ecology, sexual selection, and speciation. Ecology Letters 14:591-602.

MacArthur, R. H. 1958. Population ecology of some warblers in northeastern coniferous forests. Ecology 39:599-619.

MacArthur, R. H., Diamond, J. M., Karr, J. R. 1972. Density compensation in island faunas. Ecology 53:330-342.

MacArthur, R. H., Levins, R. 1967. The limiting similarity, convergence and divergence of coexisting species. American Naturalist 101:377-385.

MacAvoy, S. E., Arneson, L. S., Bassett, E. 2006. Correlation of metabolism with tissue carbon and nitrogen turnover rate in small mammals. Oecologia 150: 190-201. 
Magalhaes, I. S., Mwaiko, S., Schneider, M. V., Seehausen, O. 2009. Divergent selection and phenotypic plasticity during incipient speciation in Lake Victoria cichlid fish. Journal of Evolutionary Biology 22:260-27.

Mallet, J., Meyer, A., Nosil P., Feder, J. L. 2009. Space, sympatry, and speciation. Journal of Evolutionary Biology 22:2332-2341.

Manly, B. F. J. 2006. Randomization, bootstrap, and Monte Carlo methods in biology. CRC Press. London, U.K.

Mariano-Jelicich, R., Botto, F., Martineeto, P., Iribarne, O., \& Favero, M. 2008. Trophic segregation between sexes in the Black Skimmer revealed through the analysis of stable isotopes. Marine Biology 155:443-450.

Martin, R. A., Pfennig, D. W. 2009. Disruptive selection in natural populations: the roles of ecological specialization and resource competition. American Naturalist 174:268- 281.

Martin, R. A., Pfennig, D. W. 2010. Field and experimental evidence that competition and ecological opportunity promote resource polymorphism. Biological Journal of the Linnaean Society 100:73-88.

Martin, P. R., Martin, T. E. 2001. Ecological and fitness consequences of species coexistence: a removal experiment with wood warblers. Ecology 82:189-206.

Martínez del Rio, C., Anderson-Sprecher, R. 2008. Beyond the reaction variable: the meaning and significance of incorporation data. Oecologia 156:765-772. 
Martínez del Rio, C., Wolf, N., Carleton, S. A., Gannes, L. Z. 2009a. Isotopic ecology ten years after a call for more laboratory experiments. Biological Reviews of the Cambridge Philosophic Society 84:91-111.

Martínez del Rio, C., Sabat, P., Anderson-Sprecher, R., Gonzalez, S. P. 2009b. Dietary and isotopic specialization: the isotopic niche of three Cinclodes ovenbirds. Oecologia 161:149-159.

Mason, L. G. 1977. Prey preferences and ecological sexual dimorphism in Phymata americana. American Midland Naturalist 97:293-299.

Matich, P., Heithaus, M. R., Layman, C. A. 2011. Contrasting patterns of individual specialization and trophic coupling in two marine apex predators. Journal of Animal Ecology 80:294-305.

Matthews, B., Marchinko, K. B., Bolnick, D. I., Mazumder, A. 2010. Specialization of trophic position and habitat use by sticklebacks in an adaptive radiation. Ecology 91:1025-1034.

Matthews, B., Mazumder, A. 2004. A critical evaluation of intrapopulation variation of $\delta^{13} \mathrm{C}$ and isotopic evidence of individual specialization. Oecologica 140:361-371.

Maynard-Smith, J. 1966. Sympatric speciation. American Naturalist 100: 637-650.

Mayr, E. 1942. Systematics and Origins of Species. Columbia University Press, New York City, NY.

Mayr, E. 1963. Animal Species and Evolution. Belknap Press, Cambridge, MA. 
Mazerolle, M. J., Desrochers, A., Rochefort, L. 2005. Landscape characteristics influence pond occupancy by frogs after accounting for detectability. Ecological Applications $15: 824-834$.

McCaffery, R., Solonen, A., Crone, E. 2012. Frog population viability under present and future climate conditions: a Bayesian state-space approach. Journal of Animal Ecology 81:978-985.

McCallum, M. L. 2010. Future climate change spells catastrophe for Blanchard's cricket frog, Acris blanchardi (Amphibia: Anura: Hylidae). Acta Herpetologica 5:119-130.

McClelland, J. W., Montoya, J. P. 2002. Trophic relationships and the nitrogen isotopic composition of amino acids in phytoplankton. Ecology 83:2173-2180.

McKamie, J. A., Heidt, G. A. 1974. A comparison of spring food habits of the bullfrog, Rana catesbeianus, in three habitats of central Arkansas. Southwestern Naturalist 19:107-111.

McMahon, K. W., Fogel, M. L., Elsdon, T. S., Thorrold, S. R. 2010. Carbon isotope fractionation of amino acids in fish muscle reflects biosynthesis and isotopic routing from dietary protein. Journal of Animal Ecology 79:1132-1141.

Meik, J. M., Setser, K., Mociño-Deloya, E., Lawing, A. M. 2012. Sexual differences in head form and diet in a population of Mexican lance-headed rattlesnakes, Crotalus polystictus. Biological Journal of the Linnean Society 106:633-640. 
Meyer, A. 1987. Phenotypic plasticity and heterochrony in Cichlasoma managuense (Pisces, Cichlidae) and their implications for speciation in cichlid fishes. Evolution 41:1357-1369.

Michel, A. P., Rull, J., Aluja, M., Feder, J. L. 2007. The genetic structure of hawthorneinfesting Rhagoletis pomonella populations in Mexico: implications for sympatric host race formation. Molecular Ecology 16:2867-2878.

Moczek, A. P., Sultan S., Foster S., Ledón-Rettig C., Dworkin I., Nijhout H. F., Abouheif E., Pfennig, D. W. 2011. The role of developmental plasticity in evolutionary innovation. Proceedings of the Royal Society B: Biological Sciences 278:27052713.

Moore, J. W., Semmens, B. X. 2008. Incorporating uncertainity and prior information into stable isotope mixing models. Ecology Letters 11:470-480.

Morey S.R. 1990. Microhabitat selection and predation in the Pacific treefrog, Pseudacris regilla. Journal of Herpetology 24:292-296.

Morjan, C. L., Rieseberg, L. H. 2004. How species evolve collectively: implications of gene flow and selection for the spread of advantageous alleles. Molecular Ecology $13: 1341-1356$.

Murray, I.W., Wolf, B.O. 2013. Diet and growth influence carbon incorporation rates and discrimination factors $\left(\Delta^{13} \mathrm{C}\right)$ in desert box turtles, Terrapene ornata luteola. Herpetological and Conservation Biology 8:149-162. 
Murray, K. A., Skerratt, L. F., Garland, S., Kriticos, D., McCallum, H. 2013. Whether the weather drives patterns of endemic amphibian chytridiomycosis: a pathogen proliferation approach. PLoS ONE 8: e61061.

Muschick, M., Barluenga, M., Salzburger, W., Meyer, A. 2011. Adaptive phenotypic plasticity in the Midas cichlid fish pharyngeal jaw and its relevance in adaptive radiation. BMC Evolutionary Biology 11:116-128.

Nauwelaerts, S., Ramsay, S, J., Aerts, P. 2007. Morphological correlates of aquatic and terrestrial locomotion in a semi-aquatic frog, Rana esculenta: no evidence for a design conflict. Journal of Anatomy 210:304-317.

Navarro, J., Forero, M. G., González-Solís, J., Igual, J. M., Bécares, J., Hobson, K. A. 2009. Foraging segregation between two closely related shearwaters breeding in sympatry. Biology Letters 5:545-548.

Navarro, A., Ruiz, A. 1997. On the fertility effects of pericentric inversions. Genetics $147: 931-939$.

Nelson, J., Chanton, J., Coleman, F., Koenig, C. 2011. Patterns of stable carbon isotope turnover in gag, Mycteroperca microlepis, an economically important marine piscivore determined with a non-lethal surgical biopsy procedure. Environmental Biology of Fish 90:243-252.

Newell, F., Beachy, T. A., Rodewald, A. D., Rengifo, C. G., Ausprey, I. J., Rodewald, P. G. 2014. Foraging behavior of migrant warblers in mixed species flocks in 
Venezuelan shade coffee: interspecific differences, tree species selection, and effects of drought. Journal of Field Ornithology 85:134-151.

Newsome, S. D., Fogel, M. L., Kelly, L., Martínez del Rio, C. 2011. Contributions of direct incorporation from diet and microbial amino acids to protein synthesis. Functional Ecology 25:1051-1062.

Newsome, S. D., Martínez del Rio, C., Bearhop, S., Phillips, D. L. 2007. A niche for isotopic ecology. Frontiers of Ecology and the Environment 5:429-436.

Newsome, S. D., Wolf, N., Peters, J., Fogel, M. L. 2014. Amino acid $\delta^{13} \mathrm{C}$ analysis shows flexibility in the routing of dietary protein and lipids to the tissue of an omnivore. Integrative and Comparative Biology 54:890-902.

Noor, M. A. F., Grams, K. L., Bertucci, L. A., Reiland, J. 2001. Chromosomal inversions and the reproductive isolation of species. Proceedings of the National Academy of Sciences of the United States of America 98:12084-12088.

Norris, R. A. 1958. Comparative biosystematics and life history of the nuthatches Sitta pygmaea and Sitta pusilla. University of California Publications of Zoology 56:119300.

Nosil, P. 2012. Ecological speciation. Oxford University Press, Oxford, U.K.

Nosil, P., Harmon, L. J., Seehausen, O. 2009. Ecological explanations for (incomplete) speciation. Trends in Ecology and Evolution 24:145-156. 
Nosil, P., Reimchen, T. E. 2005. Ecological opportunity and levels of morphological variance within freshwater stickleback populations. Biological Journal of the Linnean Society 86:297-308.

Noske, R.A. 1986. Intersexual niche segregation among three bark-foraging birds of eucalyptus forests. Australian Journal of Ecology 11:255-267.

Nifong, J. C., Layman, C. A., Silliman, B. R. 2015. Size, sex, and individual-level behaviour drive intrapopulation variation in cross-ecosystem foraging of a toppredator. Journal of Animal Ecology 84:35-48.

O’Brien, D. M., Boggs, C. L., Fogel, M. L. 2005. The amino acids used in reproduction by butterflies: a comparative study of dietary sources using compound-specific stable isotope analysis. Physiological and Biochemical Zoology 78:819-827.

O’Brien, D. M., Fogel, M. L., Boggs, C. L. 2002. Renewable and nonrenewable resources: Amino acid turnover and allocation to reproduction in Lepidoptera. Proceedings of the National Academy of Sciences of the United States of America 99:4413-4418.

Ogden, L. J. E., Hobson, K. A., Lank, D. B. 2004. Blood isotopic $\left(\delta^{13} \mathrm{C}\right.$ and $\left.\delta^{15} \mathrm{~N}\right)$ turnover and diet-tissue fractionation factors in captive dunlin (Calidris alpina pacifica). Auk 121:170-177.

Oksanen, J. 2013. Vegan: community ecology package. R package version 2.0-9.

http://CRAN.R-project.org/package=vegan 
O’Neill, M. W., Gibb, A. C. 2014. Does feeding behavior facilitate trophic partitioning in two sympatric sucker species from the American southwest? Physiological and Biochemical Zoology 87:65-76.

Ortíz-Serrato, L., Ruiz-Campos, G., Valdez-Villavicencio, J. H. 2014. Diet of the exotic American bullfrog, Lithobates catesbeianus, in a stream of northwestern Baja California, Mexico. Western North American Naturalist 74:116-122.

Østbye, K., Amundsen, A., Bernatchez, L., Klemetsen, A., Knudsen, R., Kristoffersen, R., Næsje, T.F., Hindar, K. 2006. Parallel evolution of ecomorphological traits in the European whitefish Coregonus lavaretus (L.) species complex during postglacial times. Molecular Ecology 15, 3983-4001.

Østman, B., Hintze, A., Adami C. 2012. Impact of epistasis and pleiotropy on evolutionary adaptation. Proceedings of the Royal Society B: Biological Sciences 279:247-256.

Otto, S. P., Servedio, M. R., Nuismer, S. L. 2008. Frequency-dependent selection and the evolution of assortative mating. Genetics 179:2091-2112.

Page, B., McKenzie, J., \& Goldsworthy, S.D. (2005). Dietary resource partitioning among sympatric New Zealand and Australian fur seals. Marine Ecology Progress Series 293:283-302.

Parker, G. A. 1992. The evolution of sexual size dimorphism in fish. Journal of Fish Biology 40 (suppl. B):1-20. 
Parnell, A. C., Inger, R., Bearhop, S., Jackson, A. L. 2010 Source partitioning using stable isotopes: coping with too much variation. PLoS ONE 5: e9672.

Parent, C. E., Agashe, D., Bolnick, D. I. 2014. Intraspecific competition reduces niche width in experimental populations. Ecology and Evolution 20:3978-3990.

Parent, C. E., Crespi, B. J. 2009 Ecological opportunity in adaptive radiation of Galapagos endemic land snails. American Naturalist 174:898-905.

Parsons, K. J., Robinson, B. W. 2006. Replicated evolution of integrated plastic responses during early adaptive divergence. Evolution 60:801-813.

Pearson, D., Shine, R., How, R. 2002. Sex-specific niche partitioning and sexual size dimorphism in Australian pythons (Morelia spilota imbricata). Biological Journal of the Linnean Society 77: 113-125.

Pekár, S., Bilde. T., Martišová, M. 2011. Intersexual trophic niche partitioning in an anteating spider (Araneae: Zodariidae). PLoS ONE 6:e14603.

Penn, D. J., Damjanovich, K., Potts, W. K. 2002. MHC heterozygosity confers a selective advantage against multiple-strain infections. Proceedings of the National Academy of Sciences of the United States of America 99:11260-11264.

Pérez-Barbería, F. J., Pérez-Fernández, E., Roberston, E., Alvarez-Enríquez, B. 2008. Does the Jarman-Bell principle at the intra-specific level explain sexual segregation in polygynous ungulates? Sex differences in forage digestibility in Soay sheep. Oecologia 157:21-30. 
Perkins, S. E., Speakman, J. R. 2001. Measuring natural abundances of ${ }^{13} \mathrm{C}$ in respired $\mathrm{CO}_{2}$ : variability and implications for non-invasive dietary analysis. Functional Ecology 15:791-797.

Perry, G. 1996. The evolution of sexual dimorphism in the lizard Anolis polylepis (Iguania): evidence from intraspecific variation in foraging behavior and diet. Canadian Journal of Zoology 245:1238-1245.

Peters, D., Grubb, Jr., T.C. 1983. An experimental analysis of sex-specific foraging in the downy woodpecker, Picoides pubescens. Ecology 64:1437-1443.

Pfennig D. W., McGee M. 2010. Resource polyphenism increases species richness: a test of the hypothesis. Philosophical Transactions of the Royal Society B: Biological Sciences 365:577-591.

Pfennig D. W., Wund M. A., Snell-Rood E. C., Cruickshank T., Schlichting C. D., Moczek A. P. 2010. Phenotypic plasticity’s impacts on diversification and speciation. Trends in Ecology and Evolution 26:459-467.

Piálek, J., Hauffe, H. C., Rodríguez-Clark, K. M., Searle, J. B. 2001. Raciation and speciation in house mice from the Alps: the role of chromosomes. Molecular Ecology 10:613-625.

Pires, M. M., Guimarães, P. R., Araújo, M. S., Giaretta, A. A., Costa, J. C. L., dos Reis, S. F. 2011. The nested assembly of individual-resource networks. Journal of Animal Ecology 80:896-903. 
Pitelka, F.A. (1950). Geographic variation and the species problem in the shore-bird genus Limnodromus. University of California Publications of Zoology 50, 1-108.

Phillips, R. A., Bearhop, S., Mcgill, R. A. R., Dawson, D. A. 2009. Stable isotopes reveal individual variation in migration strategies and habitat preferences in a suite of seabirds during the nonbreeding period. Oecologia 160:795-806.

Phillips, R. A., McGill, R. A. R., Dawson, D. A., Bearhop, S. 2011. Sexual segregation in distribution, diet, and trophic level of seabirds: insights from stable isotope analysis. Marine Biology 158:2199-2208.

Phillips, D. L., Newsome, S. D., Gregg, J. W. 2005. Combining sources in stable isotope mixing models: alternative methods. Oecologia 144: 520-527.

Polito, M. J., Trivelpiece, W. Z., Karnovsky, N. J., Ng, E., Patterson, W. P., Emslie, S. D. 2011. Integrating stomach content and stable isotope analyses to quantify the diets of pygoscelid penguins. PLOS ONE 6:e26642.

Polo-Silva, C., Newsome, S. D., Galván-Magaña, Grijalba-Bendeck, M., Sanjuan-Muñoz, A. 2013. Trophic shift in the diet of the pelagic thrasher shark based on stomach contents and stable isotope analyses. Marine Biology Research 9:958-971.

Popp, B. N., Graham, B.S., Olson, R.J., Hannides, C.C.S., Lott, M.J., Lopez-Ibarra, G.A., Galvan-Magana, F., Fry, B. 2007. Insight into the trophic ecology of yellowfin tuna, Thunnus albacares, from compound-specific nitrogen isotope analysis of protenaceous amino acids. Pages 173-190 in Stable Isotopes as Indicators of Ecological Change edited by T. Dawson and R. Siegwolf. Elsevier Academic Press. 
Post, D. M. 2002. Using stable isotopes to estimate trophic position: models, methods, and assumptions. Ecology 83:703-718.

Potts, T. H. 1885. Oology of New Zealand. New Zealand Journal of Science 2:475-484.

Price, T. D., Qvarnström, A., Irwin, D. E. 2003. The role of phenotypic plasticity in driving genetic evolution. Proceedings of the Royal Society B: Biological Sciences 270:1433-1440.

Prowell, D. P., McMichael, M., Silvan, J. F. 2004. Multilocus genetic analysis of host use, introgression, and speciation in host strains of fall armyworm (Lepidoptera: Noctuidae). Annals of the Entomological Society of America 97:1034-1044.

Prout, T. 1968. Sufficient conditions for multiple niche polymorphism. American Naturalist 102:493-496.

Pulliam, H. R. 1985. Foraging efficiency, resource partitioning, and the coexistence of sparrow species. Ecology 66:1829-1836.

Pyke, G. H., Pulliam, H. R., Charnov, E. L. 1977. Optimal foraging: a selective review of theory and tests. The Quarterly Review of Biology 52:137-154.

Quesada, H., Posada, D., Caballero, A., Morán, P., Rolán-Alvarez, E. 2007. Phylogenetic evidence for multiple sympatric ecological diversification in a marine snail. Evolution 61:1600-1612.

Quevedo, M., Svanbäck, R., Eklov, P. 2009. Intrapopulation niche partitioning in a generalist predator limits food web connectivity. Ecology 90:2263-2274. 
R Core Development Team. 2013. R: a language and environment for statistical computing. R Foundation for statistical Computing, Vienna, Austria. URL http://www.R-project.org/.

Ramos, R., González-Solís, J., Forero, M. G., Moreno, R., Gómez-Díaz, E., Hobson, K. A. 2009. The influence of breeding colony and sex on mercury, selenium and lead levels and carbon and nitrogen stable isotope signatures in summer and winter feathers of Calonectris shearwaters. Oecologia 159:345-354.

Rand, A. L. 1952. Secondary sexual characters and ecological competition. FieldianaZoology 34, 65-70.

Ravingé, V., Dieckmann, U., Olivieri, I. 2009. Live where you thrive: joint evolution of habitat choice and local adaptation facilitates specialization and promotes diversity. American Naturalist 174:E141-E169.

Reum, J. C. P., Essington, T. E. 2008. Seasonal variation in guild structure of the Puget Sound demersal fish community. Estuaries and Coasts 31:790-801.

Ribera, I., Barraclough, T. G., Vogler, A. P. 2001. The effect of habitat type on speciation rates and range movements in aquatic beetles: inferences from species-level phylogenies. Molecular Ecology 10:721-725.

Rice, W. R., Salt, G. W. 1990. The evolution of reproductive isolation as a correlated character under sympatric conditions: experimental evidence. Evolution 44:11401152. 
Rice, W. R., Hostert, E. E. 1993. Laboratory experiments on speciation: what have we learned in 40 years? Evolution 47:1637-1653.

Rieseberg, L. H. 2001. Chromosomal rearrangements and speciation. Trends in Ecology and Evolution 16:351-358.

Reich, K.J., Bjorndal, K.A., Martínez del Rio, C. 2008. Effects of growth and tissue type on the kinetics of ${ }^{13} \mathrm{C}$ and ${ }^{15} \mathrm{~N}$ incorporation in a rapidly growing ectotherm. Oecologia 155: 651-663.

Rensch, B. 1960. Evolution above the species level. Columbia University Press, New York City, NY.

Robertson, R. 1971. Sexually dimorphic archaeogastropods and radulae. Annuals American Malacology Union 1970:75-78.

Rohwer, S. 1990. Foraging differences between white and dark morphs of the Pacific reef heron Egretta sacra. Ibis 132:21-26.

Rosalino, L. M., Santos, M. J., Pereira, I., Santos-Reis, M. 2009. Sex-driven differences in Egyptian mongoose's (Herpestes ichneumon) diet in its northwestern European range. European Journal of Wildlife Management 55:293-299.

Rose, L. M. 1994. Sex differences in diet and foraging behavior in white-faced capuchins (Cebus capucinus). International Journal of Primatology 15:95-114. 
Rosenblatt, A. E., Heithaus, M. R. 2011. Does variation in movement tactics and trophic interactions among American alligators create habitat linkages? Journal of Animal Ecology 80:786-798.

Rosenzweig, M. L. 1978. Competitive speciation. Biological Journal of the Linnaean Society 10:275-289.

Roth, J.D., Hobson, K.A. 2000. Stable and nitrogen isotopic fractionation between diet and tissue of captive red fox: implications for dietary reconstruction. Canadian Journal of Zoology 78:848-852.

Rothermel, B. B., Semlitsch, R. D. 2002. An experimental investigation of landscape resistance of forest versus old-field habitats to emigrating juvenile amphibians. Conservation Biology 16:1324-1332.

Rueffler, C., Van Dooren, T. J. M., Leimar, O., Abrams, P. A. 2006. Disruptive selection and then what? Trends in Ecology and Evolution 21:238-245.

Rundle, H. D., Nosil, P. 2005. Ecological speciation. Ecology Letters 8:336-352.

Ryan, M. J., Rand, A. S. 1993. Species recognition and sexual selection as a unitary problem in animal communication. Evolution 47:647-657.

Ryan P. G., Bloomer P., Moloney C. L., Grant T. J., Delport W. 2007. Ecological speciation in south Atlantic island finches. Science 315:1420-1423. 
Salvidio, S., Oneto, F., Ottonello, D., Costa, A., Romano, A. 2015. Trophic specialization at the individual level in a terrestrial generalist salamander. Canadian Journal of Zoology 93:79-83.

Sams, E., Boone, M. D. 2010. Interactions between recently metamorphosed green frogs and American toads under laboratory conditions. American Midland Naturalist 163:269-279.

Sánchez-Hernández, J., Amundsen, P. 2015. Trophic ecology of brown trout (Salmo trutta L.) in subarctic lakes. Ecology of Freshwater Fish 24:148-161.

Sargeant, B. L., Mann, J., Berggren, P., Krützen, M. 2005. Specialization and development of beach hunting, a rare foraging behavior, by bottlenose dolphins (Tursiops sp.). Canadian Journal of Zoology 83:1400-1410.

Sargeant, B. L., Mann, J. 2009. Developmental evidence for foraging traditions in wild bottlenose dolphins. Animal Behavior 78:715-721.

Schindler, D. E., Hilborn, R., Chasco, B., Boatright, C. P., Quinn, T. P., Rogers, L. A., Webster, M. S. 2010. Population diversity and the portfolio effect in an exploited species. Nature 465:609-613.

Schlichting C. D. 2004. The role of phenotypic plasticity in diversification. Pages 191200 in Phenotypic Plasticity: Functional and Conceptual Approaches edited by T. J. DeWitt and S. M. Scheiner. Oxford (United Kingdom), Oxford University Press.

Schliewen, U. K., Tautz, D., Pääbo, S. 1994. Sympatric speciation suggested by monophyly of crater lake cichlids. Nature 368:629-632. 
Schluter D. 2000. The ecology of adaptive radiations. Oxford University Press, Oxford, U.K.

Schluter, D. 2001. Ecology and the origin of species. Trends in Ecology and Evolution $16: 372-380$

Schluter, D., McPhail, J. D. 1992. Ecological character displacement and speciation in sticklebacks. American Naturalist 140:85-108.

Schluter, D., Rambaut, A. 1996. Ecological speciation in postglacial fishes [and Discussion]. Philosophical Transactions of the Royal Society B: Biological Sciences 351:807-814.

Schmidt, K., McClelland, J. W., Mente, E., Montoya, J. P., Atkinson, A., Voss, M. 2004. Trophic-level interpretation based on $\delta^{15} \mathrm{~N}$ values: implications of tissue-specific fractionation and amino acid composition. Marine Ecology Progress Series 266:4358.

Schoener, T. W. 1967. The ecological significance of sexual dimorphism in size in the lizard Anolis conspersus. Science 155:474-477.

Schriever, T. A., Williams, D. D. 2013. Ontogenetic and individual diet variation in amphibian larvae across an environmental gradient. Freshwater Biology 58:223236.

Seger, J. 1985. Intraspecific resource competition as a cause of sympatric speciation. Pages 43-53 in Evolution: essays in honor of John Maynard-Smith edited by P. J. 
Greenwood and M. Slatkin. Cambridge (United Kingdom): Cambridge University Press.

Selander, R. K. 1966. Sexual dimorphism and differential niche utilization in birds. The Condor 68:113-151.

Seminoff, J. A., Bjorndal, K. A., Bolten, A. B. 2007. Stable carbon and nitrogen isotope discrimination and turnover in pond sliders Trachemys scripta: insights for trophic study of freshwater turtles. Copeia 2007: 534-542.

Seminoff, J. A., Jones, T. T., Eguchi, T., Hastings, M., Jones, D. R. 2009. Stable carbon and nitrogen isotope discrimination in soft tissues of the leatherback turtle (Dermochelys coriacea): insights for trophic studies of marine turtles. Journal of Experimental Marine Biology and Ecology 381:33-41.

Semlitsch, R. D., Harris, R. N., Wilbur, H. M. 1990. Paedomorphosis in Ambystoma talpoideum: maintenance of population variation and alternative life-history pathways. Evolution 44:1604-1613.

Servedio, M. R., Doorn, G. S. V., Kopp, M., Frame, A. M., Nosil, P. 2011. Magic traits in speciation: "magic" but not rare? Trend in Ecology and Evolution 26:389-397.

Sharpe, D. M. T., Chapman, L. J. 2014. Niche expansion in a resilient endemic species following introduction of a novel top predator. Freshwater Biology 59:2539-2554.

Shepard, D. B. 2002. Spatial relationships of male green frogs (Rana clamitans) throughout the activity season. American Midland Naturalist 148:394-400. 
Shine, R. 1989. Ecological causes for the evolution of sexual dimorphism: a review of the evidence. The Quarterly Review of Biology 64:419-461.

Shine, R. 1991. Intersexual dietary divergence and the evolution of sexual dimorphism in snakes. American Naturalist 138:103-122.

Shine, R. M., Olsson, M., Lemaster, M. P., Greene, M., Mason, R. T. 2000. Body size enhances mating success in male garter snakes. Animal Behavior 59:F4-F11.

Siepielski, A. M., McPeek, M. A. 2010. On the evidence for species coexistence: a critique of the coexistence program. Ecology 91:3153-3164.

Simard F., Ayala D., Kamdem G. C., Pombi M., Etouna J., Ose K., Fotsing J. M., Fontenille D., Besansky N. J., Costantini C. 2009. Ecological niche partitioning between Anopheles gambiae molecular forms in Cameroon: the ecological side of speciation. BMC Ecology 9:17.

Skúlason, S., Smith, T. B. 1995. Resource polymorphisms in vertebrates. Trends in Ecology and Evolution 10:366-370.

Skúlason, S., Snorrason, S., Jónsson, B. 1999. Sympatric morphs, populations, and speciation in freshwater with an emphasis on Arctic charr. Pages 70-92 in Evolution of Biological Diversity edited by A. E. Magurran and R. M. May. Oxford (United Kingdom): Oxford University Press.

Slatkin, M. 1984. Ecological causes of sexual dimorphism. Evolution 38:622-630.

Smadja, C. M., Butlin, R. K. 2011. A framework for comparing processes of speciation in 
the presence of gene flow. Molecular Ecology 20:5123-5140.

Smith, P.C., Evans, P.R. 1973. Studies of shore-birds at Lindisfarne, Northumberland. I. Feeding ecology and behavior of the bar-tailed godwit. Wildfowl 24, 135-139.

Smith, T. B. 1993. Disruptive selection and the genetic basis of bill size polymorphism in the African finch Pyrenestes. Nature 363:618-620.

Smith, T. B., Skúlason, S. 1996. Evolutionary significance of resource polymorphisms in fishes, amphibians, and birds. Annual Review of Ecology and Systematics 27:111133.

Solé, M. O., Beckmann B., Kwet, A. P., Engels, W. 2005. Stomach-flushing for diet analysis in anurans: an improved protocol evaluated in a case study in Araucaria forests, southern Brazil. Studies in Neotropical Fauna and Environment 40:23-28.

Staniland, I. J. 2005. Sexual segregation in seals. Pages 53-73 in Sexual segregation in vertebrates: ecology of the two sexes, edited by K. Ruckstuhl and P. Neuhaus. Cambridge (United Kingdom): Cambridge University Press.

Staniland, I. J., Robinson, S. L. 2008. Segregation between the sexes: Antarctic fur seals, Arctocephalus gazella, foraging at South Georgia. Animal Behavior 75:1581-1590.

Stegall, V. K., Farley, S. D., Rea, L. D., Pitcher, K. W., Rye, R. O., Kester, C. L., Stricker, C. A., Bern, C. R. 2008. Discrimination of carbon and nitrogen isotopes from milk to serum and vibrissae in Alaska Steller sea lions (Eumetopias jubatus). Canadian Journal of Zoology 86:17-23. 
Steinfartz, S., Weitere, M., Tautz, D. 2007. Tracing the first step to speciation: ecological and genetic differentiation of a salamander population in a small forest. Molecular Ecology 16:4550-4561.

Stephens, P. A., Buskirk, S. W., Hayward, G. D., Martínez del Rio, C. 2005. Information theory and hypothesis testing: a call for pluralism. Journal of Applied Ecology 42:412.

Stephens, P. R., Wiens, J. J. 2009. Evolution of sexual size dimorphisms in emydid turtles: ecological dimorphism, Rensch's rule, and sympatric divergence. Evolution $63: 910-925$

Storer, R.W. 1952. Variation in the resident sharp-shinned hawks of México. Condor 54:283-289.

Streisfeld, M. A., Rausher, M. D. 2010. Population genetics, pleiotropy, and the preferential fixation of mutations during adaptive evolution. Evolution 65:629-642.

Stuart, S. N., Chanson, J. S., Cox, N. A., Young, B. E., Rodrigues, A. S. L., Fischman, D. L., Waller, R. W. 2004. Status and trends of amphibian declines and extinctions worldwide. Science 306:1783-1786.

Sultan, S. E. 2000. Phenotypic plasticity for plant development, function, and life history. Trends in Plant Science 5:537-542.

Suring, E., Wing, S. R. 2009. Isotopic turnover rate and fractionation in multiple tissues of red rock lobster (Jasus edwardsii) and blue cod (Parapercis colias): consquences 
for ecological studies. Journal of Experimental Marine Biology and Ecology 370:56-63.

Svanbäck, R., Bolnick, D. I. 2005. Intraspecific competition affects the strength of individual specialization: an optimal diet theory method. Evolutionary Ecology Research 7:993-1012.

Svanbäck, R., Bolnick, D. I. 2007. Intraspecific competition drives increased resource use diversity within a natural population. Proceedings of the Royal Society B: Biological Sciences 274:839-844.

Svanbäck, R., Eklöv, P. 2006. Genetic variation and phenotypic plasticity: causes of morphological and dietary variation in Eurasian perch. Evolution Ecology Research 8:37-49.

Svanbäck, R., Persson, L. 2004. Individual diet specialization, niche width and population dynamics: implications for trophic polymorphisms. Journal of Animal Ecology 73:973-982.

Svanbäck, R., Pineda-Krch, M., Doebeli, M. 2009. Fluctuating population dynamics promotes the evolution of phenotypic plasticity. American Naturalist 174:176-189.

Svanbäck, R., Rydberg, C., Leonardsson, K., Englun, G. 2011. Diet specialization in a fluctuating population of Saduria entomon: a consequence of resource or forager densities? Oikos 120:848-854. 
Symes, C.T., Wilson, J. W., Woodborne, S. M., Shaikh, Z. S., Scantlebury, M. 2013. Resource partitioning of sympatric small mammals in an African forest-grassland vegetation mosaic. Austral Ecology 38: 721-729.

Taylor, E. B., McPhail, J. D. 2000. Historical contingency and ecological determinism interact to prime speciation in stickleback, Gasterosteus aculeatus. Proceedings of the Royal Society B: Biological Sciences 267:2375-2385.

Temeles, E. J. 1985. Sexual size dimorphism of bird-eating hawks: the effect of prey vulnerability. American Naturalist 125:485-499.

Temeles, E. J., Koulouris, C. R., Sander S. E., Kress, W. J. 2009. Effect of flower shape and size on foraging performance and trade-offs in a tropical hummingbird. Ecology 90:1147-1161.

Temeles, E. J., Miller, J. S., Rifkin, J. L. 2010. Evolution of sexual dimorphism in bill size and shape of hermit hummingbirds (Phaethornithinae): a role for ecological causation. Philosophical Transactions of the Royal Society B: Biological Sciences 365:1053-1063.

Temeles, E. J., Pan, I. L., Brennan, J. L., Horwitt, J. N. 2000. Evidence for ecological causation of sexual dimorphism in a hummingbird. Science 289:441-443.

Tieszen, L. L., Boutton, T. W., Tesdahl, K. G., Slade, N. A. 1983. Fractionation and turnover of stable carbon isotopes in animal tissues: implications for $\delta^{13} \mathrm{C}$ analysis of diet. Oecologia 57:32-37. 
Thibert-Plante, X., Gavrilets, S. 2013. Evolution of mate choice and the so-called magic traits in ecological speciation. Ecology Letters 16:1004-1013.

Thibert-Plante X., Hendry A. P. 2011a. The consequences of phenotypic plasticity for ecological speciation. Journal of Evolutionary Biology 24:326-342.

Thibert-Plante, X., Hendry, A. P. 2011b. Factors influencing progress towards sympatric speciation. Journal of Evolutionary Biology 24:2186-2196.

Thom, M. D., Harrington, L. A., Macdonald, D. W. 2004. Why are American mink sexually dimorphic? A role for niche separation. Oikos 105:525-535.

Tinker, T. M., Bentall, G., Estes, J. A. 2008. Food limitation leads to behavioral diversification and dietary specialization in sea otters. Proceedings of the National Academy of Sciences of the United States of America 105:560-565.

Tinker, T. M., Guimarães Jr., P. R., Novak, M., Marquitti, F. M. D., Bodkin, J. L., Staedler M., Bentall, G., Estes, J. A. 2012. Structure and mechanism of diet specialization: testing models of individual variation in resource use with sea otters. Ecology Letters 15:475-483.

Toft, C. A. 1980. Feeding ecology of thirteen syntopic species of anurans in a seasonal tropical environment. Oecologia 45:131-141.

Toft, C. A. 1985. Resource partitioning in amphibians and reptiles. Copeia 1985:1-21. 
Trakimas, G., Jardine, T.D., Barisevičiūtè, R., Garbaras, A., Skipitytè, R., Remeikis, V. 2011. Ontogenetic dietary shifts in European common frog (Rana temporaria) revealed by stable isotopes. Hydrobiologia 675:87-95.

Trickett, A. J., Butlin, R. K. 1994. Recombination suppressors and the evolution of new species. Heredity 73:339-345.

Trivers, R. L. 1976. Sexual selection and resource-accruing abilities in Anolis garmani. Evolution 30:253-269.

Tucker, S., Bowen, W. D., Iverson, S. J. 2007. Dimensions of diet segregation in grey seals Halichoerus grypus revealed through stable isotopes of carbon $\left(\mathrm{d}^{13} \mathrm{C}\right)$ and nitrogen $\left(\mathrm{d}^{15} \mathrm{~N}\right)$. Marine Ecology Progress Series 339:271-282.

Tucker, S., Bowen, W. D., Iverson, S. J., Stenson, G. B. 2009. Intrinsic and extrinsic sources of variation in the diets of harp and hooded seals revealed by fatty acid profiles. Canadian Journal of Zoology 87:139-151.

Van de Pol, M., Brouwer, L., Ens, B. J., Oosterbeek, K., Tinbergen, J. M. 2009. Fluctuating selection and the maintenance of individual and sex-specific diet specialization in free-living oystercatchers. Evolution 64:836-851.

Van Dooren, T. J. M. 2006. Protected polymorphism and evolutionary stability in pleiotropic models with trait-specific dominance. Evolution 60:1991-2003.

Van Valen, L. M. 1965. Morphological variation and width of ecological width. American Naturalist 99:377-390. 
Vander Zanden, M. J., Rasmussen, J. B. 2001. Variation in $\delta^{15} \mathrm{~N}$ and $\delta^{13} \mathrm{C}$ trophic fractionation: implications for aquatic food web studies. Limnology and Oceanography 46:2061-2066.

Vanderklift, M. A., Ponsard, S. 2003. Sources of variation in consumer-diet $\delta^{15} \mathrm{~N}$ enrichment: a meta-analysis. Oecologia 136:169-182.

Via, S. 2001. Sympatric speciation in animals: the ugly duckling grows up. Trends in Ecology and Evolution 16:381-390.

Wallace, A. R. 1889. Darwinism: an exposition of the theory of natural selection with some of its application. MacMillan Press, London, U. K.

Walsh, B. J. 1864. On phytophagous varieties and phytophagous species. Proceedings of the Entomological Society of Philadelphia 3 403-430.

Wang, Y., Guo, Z., Pearl, C. A., Li, Y. Body size affects the predatory interactions between introduced American Bullfrogs (Rana catesbeiana) and native anurans in China: an experimental study. Journal of Herpetology 41:514-520.

Ward, P. 1965. Feeding ecology of the black-faced dioch Quelea quelea in Nigeria. Ibis 107:173-214.

Warne, R. W., Gilman, C. A., Wolf, B. O. 2010. Tissue-carbon incorporation rates in lizards: implications for ecological studies using stable isotopes in terrestrial ectotherms. Physiological and Biochemical Zoology 83: 608-617. 
Wells, K. D. 2007. The ecology and behavior of amphibians. University of Chicago Press. Chicago, U.S.A.

Wennersten, L., Forsman, A. 2012. Population-level consequences of polymorphism, plasticity and randomized phenotype switching: a review of predictions. Biological Reviews of the Cambridge Philosophical Society 87:756-767.

Werner, E. E. 1994. Direct and indirect effects of predators on two anuran species along and environmental gradient. Ecology 75:1368-1382.

Werner, E. E., Wellborn, G. A, McPeek, M. A. 1995. Diet composition in postmetamorphic bullfrogs and green frogs: implications for interspecific predation and competition. Journal of Herpetology 29:600-607.

West-Eberhard M. J. 2003. Developmental Plasticity and Evolution. New York (United States of America): Oxford University Press.

Whiting, A. R. 1967. The biology of the parasitic wasp Mormoniella vitripennis (= Nasiconia brevicornis) (Walker). Quarterly Review of Biology 42:333-406.

Williamson, P. 1971. Feeding ecology of the red-eyed vireo (Vireo olivaceus) and associated foliage gleaning birds. Ecological Monographs 41, 129-152.

Wolf, J. B. W., Harrod, C., Brunner, S., Salazar, S., Trillmich, F., Tautz, D. 2008. Tracing early stages of species differentiation: ecological, morphological and genetic divergence of Galapagos sea lion populations. BMC Evolutionary Biology $8: 150$ 
Wooton, R. J., Adams, C. E., Attrill, M. J. 2005. Empirical modeling of the population dynamics of a small population of the threespined stickleback, Gasterosteus aculeatus. Environmental Biology of Fishes 74:151-161.

Wund, M. A., Valena, S., Wood, S., Baker, J. A. 2012. Ancestral plasticity and allometry in threespined stickleback reveal phenotypes associated with derived, freshwater ecotypes. Biological Journal of the Linnean Society 105:573-583.

Yoder, J. B., Clancey, E., des Roches, S., Eastman, J. M., Gentry, L., Godsoe, W., Hagey, T. J., Jochimsen, D., Oswalk, B. P., Robertson, J., Sarver, B. A. J., Schenks, J. J., Spear, S. F., Harmon, L. J. 2010. Ecological opportunity and the origin of adaptive radiations. Journal of Evolutionary Biology 23:1581-1596.

Young, H. S., McCauley, D. J., Dirzo, R., Dunbar, R. B., Shaffer, S.A. 2010. Niche partitioning among and within sympatric tropical seabirds revealed by stable isotope analysis. Marine Ecology Progress Series 416:285-294.

Zamella, R. A., Bunnell J. F. 2000. The distribution of anurans in two river systems of a coastal plain watershed. Journal of Herpetology 34:210-221.

Zavalaga, C.B., Halls, J.N., Mori, G.P., Taylor, S.A. \& Dell-Omo, G. 2008. Foraging areas of breeding Blue-footed Boobies Sula nebouxii in northern Peru, as determined by direction recorder. Marine Ecology Progress Series 404:259-274.

Zhao, T., Villéger, S., Lek, S., Cucherousset, J. 2014. High intraspecific variability in the functional niche of a predator is associated with ontogenetic shift and individual specialization. Ecology and Evolution 4:4649-4657. 


\title{
CURRICULUM VITAE
}

\author{
Carl S. Cloyed \\ Biology Department \\ 139 Life Sciences Building \\ University of Louisville \\ Louisville, KY 40208 \\ Work: (502)-852-7730, Cell: (970)-309-4396 \\ cscloy02@louisville.edu \\ Website: www.carlscloyed.com
}

\section{EDUCATION:}

2009-Present

Ph.D. candidate, University of Louisville, Biology

2004-2008

B.A., Prescott College, Ecology and Creative Writing

\section{RESEARCH INTERESTS:}

- Eco-evolutionary dynamics

- Intrapopulation niche variation

- Evolution of resource polymorphisms

- Community assembly and species coexistence

\section{FELLOWSHIPS AND AWARDS:}

2014 Dissertation Completion Award 
2012-2013

2012

\section{GRANTS}

2013

2013

2011

2011

2011

2010

2010
Horner Reserve Research Fellowship

Best Presentation in Ecology: Kentucky Academy of Sciences
Research Initiation Grant, University of Louisville

Graduate Student Council Research Fund, University of Louisville

Kentucky Society of Natural History, Woody Beobinger Award

Horner Reserve Research Fund

Graduate Student Union Research Grant, University of Louisville

Kentucky Society of Natural History, Bernadine Meyer Memorial Award

Creative Activities and Research Grant, University of Louisville

\section{PUBLICATIONS:}

Cloyed, C.S. and Eason, P.K. 2015. Night and day: comparing flight initiation dynamics in two closely related species of true frogs. J. Zool.295: 206-213.

Cloyed, C.S. 2014. Forest structure affects resource partitioning between pygmy and white-breasted nuthatches. Coevolution 2: 26-30.

In review/preparation (ask to see)

Cloyed, C.S., Newsome, S.D., and Eason, P.K. (In revision). Trophic discrimination values and isotopic incorporation rates in adult green frogs, Lithobates clamitans. Physiol.Biochem. Zool.

Cloyed, C.S. and Eason, P.K. (In prep). Resource partitioning and the role of intrapopulation niche variation in structuring a guild of generalist anurans. J. Anim. Ecol. 
Cloyed, C.S., and Eason, P.K. (In prep). Different ecological conditions support individual specialization in closely related, ecologically similar species

Cloyed, C.S. and Eason, P.K. (In prep). The effects of population diversity on sympatric speciation. Q. Rev. Biol.

\section{INVITED SEMINARS}

2015 Cloyed, C.S. Using stable isotopes to determine resource partitioning and the role of intrapopulation niche variation in structuring a guild. National Great Rivers Research and Education. East Alton, IL. U.S.A.

\section{SCIENTIFIC PRESENTATIONS:}

2014 Cloyed, C.S. and Eason, P.K. Individual specialization among a guild of generalist predators. Ecological Society of America $99^{\text {th }}$ Annual Meeting, Sacramento, CA. U.S.A.

2014 Cloyed, C.S. and Eason, P.K. Trophic discrimination factors and isotopic incorporation rates in adult green frogs, Lithobates clamitans. Joint Meeting of Ichthyologists and Herpetologists, Chattanooga, TN. U.S.A.

2014 Cloyed, C.S. and Eason, P.K. Using stomach content analysis and stable isotope analysis to determine diets of adult anurans. Joint Meeting of Ichthyologists and Herpetologists, Chattanooga, TN. U.S.A.

2013 Cloyed, C.S., Newsome, S.D., and Eason, P.K. Populations and communities affect individual dietary patterns of bullfrogs, Lithobates catesbeianus. Ecological Society of America $98^{\text {th }}$ Annual Meeting, Organized Oral Session. Minneapolis, MN, U.S.A.

2013 Cloyed, C.S. and Eason, P.K. The proximate and ultimate causes of individual diet variation in bullfrogs, Lithobates catesbeianus. 2013 Joint Evolution Meeting. Snowbird, UT, U.S.A.

2013 Cloyed, C.S. and Eason, P.K. Flight initiation dynamics of two true frogs, Lithobates catesbeiana and L. clamitans. Annual Animal Behavior Conference, Bloomington, IN, U.S.A. 
2012 Cloyed, C.S., Newsome, S.D., and Eason, P.K. Individual diet variation in anurans: who and why. Kentucky Academy of Sciences. Richmond, KY. U.S.A.

2012 Cloyed, C.S., Newsome, S.D., and Eason, P.K. Inter- and intraspecific diet variation using stable isotopes in three species of Anura. International Conference for the Application of Stable Isotope Techniques to Ecological Studies. Brest, FR.

\section{PUBLIC PRESENTATIONS:}

2013 Cloyed, C.S. Amphibians and prey selection at Bernheim Arboretum and Research Forest. Bernheim Arboretum and Research Forest. Clermont, KY, U.S.A.

2011 Cloyed, C.S. Secret specialists: how Kentucky's frogs may share resources. Kentucky Society of Natural History. Carter Cave State Park, KY, U.S.A.

\section{TEACHING EXPERIENCE}

Guest Lecturer: University of Louisville

2014/2015 Comparative Vertebrate Anatomy: Muscles

2013 Population and Community Ecology: Food Webs

\section{Graduate Teaching Assistant: University of Louisville}

2013-Present Human Anatomy and Physiology I \& II

2010-2012 Comparative Vertebrate Anatomy Laboratory

2009-2012 Introduction to Biological Sciences Laboratory

\section{Undergraduate Research Mentoring (*Non-thesis Masters student)}

2012-2013

2012

2012

2011
Using stable isotopes to measure frog diets

Quantifying individual diet variation in frogs

Quantifying individual diet variation in toads

*Comparing frog stomach contents to environmental abundances 


\section{Undergraduate Teaching Assistant and Tutor: Prescott College} 2008 Introduction to Writing Workshop 2007-2008 Tutor: Calculus, Statistics, Chemistry, Biology

Tutor: Yavapai Community College

2008-2009

English, Math, Chemistry, Biology

\section{SOCIETIES, COMMITTEES, AND WORKSHOPS}

2014

2012-2014

2013

2010-2013

2013

2013

2013

2012

2012

2011-2012

2010
Applying for NSF Grants for Ecological Studies. ESA Workshop

President: Biology Graduate Student Association

Phylogenetics Workshop. Joint Evolution Meeting

Ecological Society of America

American Society of Naturalists

Society for the Study of Amphibians and Reptiles

Biology Graduate Student Association Grant Selection Committee

Graduate Student Union Grant Selection Committee

Sampling Animal Tissues for Stable Isotope Analysis Workshop. $8^{\text {th }}$ ISOECOL conference

Graduate Teaching Assistant Academy

Using Mixing Models in Stable Isotope Analysis Workshop. $7^{\text {th }}$ ISOECOL conference 